\title{
Inferring Lower Runtime Bounds for Integer Programs
}

\author{
FLORIAN FROHN, Max Planck Institute for Informatics, Germany \\ MATTHIAS NAAF, RWTH Aachen University, Germany \\ MARC BROCKSCHMIDT, Microsoft Research, UK \\ JÜRGEN GIESL, LuFG Informatik 2, RWTH Aachen University, Germany
}

We present a technique to infer lower bounds on the worst-case runtime complexity of integer programs, where in contrast to earlier work, our approach is not restricted to tail-recursion. Our technique constructs symbolic representations of program executions using a framework for iterative, under-approximating program simplification. The core of this simplification is a method for (under-approximating) program acceleration based on recurrence solving and a variation of ranking functions. Afterwards, we deduce asymptotic lower bounds from the resulting simplified programs using a special-purpose calculus and an SMT encoding. We implemented our technique in our tool LoAT and show that it infers non-trivial lower bounds for a large class of examples.

CCS Concepts: • Theory of computation $\rightarrow$ Complexity classes; Program analysis; Automated reasoning; • Software and its engineering $\rightarrow$ Software performance;

Additional Key Words and Phrases: Integer programs, runtime complexity, lower bounds, automated complexity analysis

\section{ACM Reference format:}

Florian Frohn, Matthias Naaf, Marc Brockschmidt, and Jürgen Giesl. 2020. Inferring Lower Runtime Bounds for Integer Programs. ACM Trans. Program. Lang. Syst. 42, 3, Article 13 (October 2020), 50 pages.

https://doi.org/10.1145/3410331

\section{INTRODUCTION}

Recent advances in program analysis yield efficient methods to find upper bounds on the complexity of sequential integer programs. Here, one usually considers "worst-case complexity", i.e., for any variable valuation, one analyzes the length of the longest execution starting from that valuation. But in many cases, in addition to upper bounds, it is also important to find lower bounds for this notion of complexity. Together with an analysis for upper bounds, this can be used to infer tight complexity bounds. Lower bounds also have important applications in security analysis. If

This work is funded by the Deutsche Forschungsgemeinschaft (DFG, German Research Foundation, https://www.dfg.de/) 389792660 as part of TRR 248 (https://perspicuous-computing.science) and by the Deutsche Forschungsgemeinschaft (DFG, German Research Foundation, https://www.dfg.de/) - 235950644 (Project GI 274/6-2).

Authors' addresses: F. Frohn, Max Planck Institute for Informatics, Saarland Informatics Campus, Campus E1 4, 66123 Saarbrücken, Germany; email: florian.frohn@mpi-inf.mpg.de; M. Naaf, Mathematische Grundlagen der Informatik, RWTH Aachen University, 52056 Aachen, Germany; email: naaf@logic.rwth-aachen.de; M. Brockschmidt, Microsoft Research, 21 Station Road, Cambridge CB1 2FB, United Kingdom; email: mabrocks@microsoft.com; J. Giesl, LuFG Informatik 2, RWTH Aachen University, Ahornstr. 55, 52074 Aachen, Germany; email: giesl@informatik.rwth-aachen.de.

Permission to make digital or hard copies of part or all of this work for personal or classroom use is granted without fee provided that copies are not made or distributed for profit or commercial advantage and that copies bear this notice and the full citation on the first page. Copyrights for third-party components of this work must be honored. For all other uses, contact the owner/author(s).

(C) 2020 Copyright held by the owner/author(s).

0164-0925/2020/10-ART13

https://doi.org/10.1145/3410331 
one can infer that there exists a family of inputs that lead to unacceptably large runtime of the program (i.e., a family for which there is a non-linear or probably even exponential lower bound on the runtime), then this family of inputs represents a possible denial-of-service attack. Thus, techniques for the computation of lower bounds on the worst-case complexity can be used to detect such attacks. ${ }^{1}$

While worst-case lower bounds are useful to detect attacks or performance bugs, worst-case upper bounds can prove the absence of such problems. Best-case lower bounds, which have also been investigated in the literature (see Section 7), are bounds on all program runs, whereas worst-case lower bounds hold for (usually infinite) families of (expensive) program runs. Thus, best-case lower bounds can, e.g., be used to decide whether a certain task is expensive enough to compensate the overhead of executing it remotely. So in general, the use cases of worst-case and best-case bounds are orthogonal.

We introduce the first technique to infer worst-case lower bounds for integer programs automatically. Besides concrete bounds, our technique can also deduce asymptotic bounds. In general, concrete lower bounds that hold for arbitrary variable valuations are difficult to express concisely. In contrast, asymptotic bounds are easily understood by humans and witness possible attacks in a convenient way.

We first introduce our program model in Section 2. Afterwards, Section 3 shows how to transform arbitrary tail-recursive integer programs into so-called simplified programs without loops. To this end, in Section 3.1, we introduce a variation of classical ranking functions that we call metering functions. These metering functions are used to under-estimate the number of iterations of simple loops, i.e., loops consisting of a single transition without nested loops or branching. Based on this concept, we present a framework to simplify programs iteratively in Sections 3.2 and 3.3. It transforms tail-recursive programs (with branching and sequences of possibly nested loops) into programs without loops. To this end, it accelerates and then eliminates simple loops by (under-)approximating their effect using a combination of metering functions and recurrence solving. In Section 4, we extend our technique to an automatic approach that also transforms nontail-recursive integer programs into simplified programs.

Section 5 introduces techniques that allow us to infer asymptotic lower bounds from simplified programs. In Sections 5.1 and 5.2, we present a calculus to compute asymptotic bounds by repeatedly simplifying a limit problem, which is an abstraction of the path condition $\varphi$ of a simplified program. This abstraction allows us to focus on $\varphi$ 's behavior in the limit, i.e., we search for an infinite family of inputs that satisfy $\varphi$. In addition, Section 5.3 shows how a limit problem can be encoded into a quantifier-free first-order formula with integer arithmetic. Then off-the-shelf SMT solvers can be used to find a model for this formula. This model in turn gives rise to the desired family of inputs that satisfy $\varphi$. Thus, in many cases, we can benefit from the power of SMT solvers instead of applying the rules of our calculus from Section 5.2 heuristically. Note that the calculus from Section 5.2 can simplify limit problems such that our SMT encoding from Section 5.3 becomes applicable; and moreover our SMT encoding can be integrated into the calculus from Section 5.2, i.e., both techniques complement each other.

Finally, we evaluate our implementation in the tool LoAT in Section 6, discuss related work in Section 7, and conclude in Section 8.

A preliminary version of this article was published in the conference paper [29]. The current article extends our earlier paper [29] significantly with the following novel contributions:

\footnotetext{
${ }^{1}$ In a joint project $C A G E[23,28]$ with Draper Inc. (https://www.draper.com) and the University of Innsbruck, we used our tool LoAT (which implements the techniques described in this article) together with our tool KoAT [14] (which infers upper runtime bounds) to analyze the complexity of large Java programs to detect vulnerabilities.
} 
(1) The new Theorem 3.4 integrates an optimization that we proposed in the paper [29] into our notion of metering functions. The novel insight is that in this way we can infer more expressive "conditional" metering functions of the form $\llbracket \psi \rrbracket \cdot b$ where $b$ is an ordinary arithmetic expression and $\llbracket \psi \rrbracket$ is the characteristic function of the arithmetic condition $\psi$ (i.e., $\llbracket \psi \rrbracket$ yields 1 if $\psi$ is satisfied and 0 , otherwise). Such metering functions are also useful to treat terminating and non-terminating rules in a uniform way in our approach. To ease the use of such metering functions, in Theorem 3.8, we extend the technique for accelerating loops from our paper [29, Theorem 7] accordingly. Thus, conditional metering functions are now seamlessly integrated into our framework, resulting in a more streamlined formalization and presentation than in our previous paper [29].

(2) In Theorem 3.12, we present a technique to eliminate variables from the program. To apply it automatically, the new Lemma 3.15 clarifies how to check a crucial side condition that requires that certain arithmetic expressions evaluate to integers whenever one instantiates their variables with integers. A similar side condition is also needed for the automation of our calculus for limit problems in Section 5. Moreover, this check could also be used to ensure that the initial program is "well formed" before starting the analysis. In our earlier paper [29], the automation of this check has not been discussed.

(3) We lift our approach to non-tail-recursive programs in the new Section 4. In contrast, the paper [29] was restricted to tail-recursive integer programs. While we used a graphbased program model before [29], in the current article, we propose a different (rule-based) representation of integer programs. This representation allows an easy formulation of non-tail-recursion by using rules whose right-hand sides are multisets of terms.

(4) The SMT encoding from Section 5.3 is completely new and improves the performance of our approach considerably. In particular, in our experiments of Section 6 it outperformed the calculus from Section 5.2 on examples with polynomial limit problems.

(5) We provide formal proofs for all lemmas and theorems, which were missing in our paper [29].

(6) Throughout the article, we added many more examples, discussions, and explanations.

\section{PROGRAM MODEL}

We consider sequential imperative integer programs, allowing non-linear arithmetic and nondeterminism, whereas heap usage and concurrency are not supported. In earlier work [14], we used an equivalent program model and showed how to deduce upper runtime bounds for integer programs.

Most existing abstractions that transform heap programs to integer programs are "overapproximations". However, to apply our approach to heap programs, we would need an underapproximating abstraction to ensure that the inference of worst-case lower bounds is sound. As in most related work, we treat numbers as mathematical integers $\mathbb{Z}$. However, one can use suitable transformations $[24,42]$ to handle machine integers correctly, e.g., by inserting explicit normalization steps at possible overflows.

In our program model, we use a rule-based representation of integer programs where integer program rules are of the form $f(\bar{x}) \stackrel{c}{\rightarrow} T[\varphi]$. The left-hand side $f(\bar{x})$ consists of a function symbol $f$ and a vector of pairwise different variables $\bar{x}$. The sets of all function symbols and all variables are $\Sigma$ and $\mathcal{V}$, respectively. While $\Sigma$ is finite, we assume $\mathcal{V}$ to be countably infinite, as we rely on fresh variables to model non-determinism. The arithmetic expression $c$ represents the cost of the rule, where arithmetic expressions are composed of variables from $\mathcal{V}$, numbers, and pre-defined operations like,,$+- *$, and so on. Annotating rules with costs enables a modular analysis, as it allows us to summarize a sub-program $\mathcal{P}$ into a single rule whose cost is a lower bound on $\mathcal{P}$ 's 
complexity. To ease readability, we sometimes omit the costs of rules. The guard $\varphi$ is a constraint over $\mathcal{V}$, i.e., a finite conjunction ${ }^{2}$ of inequations (built with $<, \leq,>$, or $\geq$ ) over arithmetic expressions, which we omit if it is empty (i.e., we write $f(\bar{x}) \stackrel{c}{\rightarrow} T$ instead of $f(\bar{x}) \stackrel{c}{\rightarrow} T$ [true]). So $c$ is an expression like $x \cdot y+2^{y}$ and $\varphi$ is a formula like $x \cdot y \leq 2^{y} \wedge y>0$, for example. The right-hand side $T$ is a multiset of terms of the form $g(\bar{t})$ where $g \in \Sigma$ and $\bar{t}$ is a vector of arithmetic expressions. In the following, the notion of "term" always refers to terms of this specific form. The set of all terms is $\mathcal{T}$. We use $\mathcal{V}(\cdot)$ to denote all variables occurring in the argument expression (e.g., $\mathcal{V}(t)$ consists of all variables occurring in the term $t$ ).

Note that as in the paper [14], we do not allow nested calls of function symbols from $\Sigma$ in righthand sides of rules. For that reason, our program model also does not support return values. Instead of a rule $f(\bar{x}) \stackrel{c}{\rightarrow} f(g(\bar{t}))[\varphi]$ with $f, g \in \Sigma$, one has to represent the result of the inner call $g(\bar{t})$ by a fresh temporary variable $t v$. So one uses a rule $f(\bar{x}) \stackrel{c}{\rightarrow}\{g(\bar{t}), f(t v)\}[\varphi \wedge \psi]$ instead, where $\psi$ may restrict the possible values of $t v$ by suitable inequations.

We say that a function symbol $f$ has an incoming rule $\alpha$ if $f$ occurs in $\alpha$ 's right-hand side and $f$ has an outgoing rule $\alpha$ if $f$ occurs on (the root position of) $\alpha$ 's left-hand side. Given a rule $\alpha$, $\operatorname{root}(\alpha)$ denotes the root symbol of $\alpha$ 's left-hand side $\operatorname{lhs}(\alpha)$. Furthermore, $\operatorname{cost}(\alpha)$, $\operatorname{rhs}(\alpha)$, and $\operatorname{guard}(\alpha)$ denote the cost, the right-hand side, and the guard of $\alpha$. The number of elements of the multiset in $\alpha$ 's right-hand side is called the degree of $\alpha$. A rule is tail-recursive if its degree is at most $1 .{ }^{3}$ For a rule $\alpha$ with degree 1 , let target $(\alpha)$ be the root symbol of the term in $\operatorname{rhs}(\alpha)$.

An integer program is a finite set of integer program rules. It is tail-recursive if all of its rules are tail-recursive.

Example 2.1 (Fibonacci). Consider the following imperative program, which computes the xth Fibonacci number and returns 1 if $\mathrm{x}$ is negative.

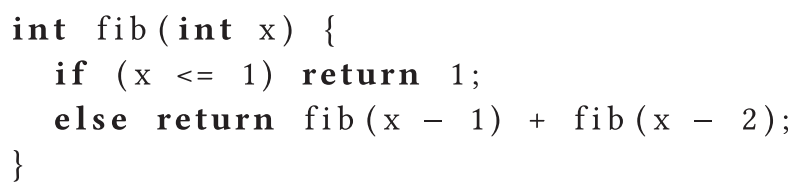

A suitable abstraction of this program would yield the following integer program, which represents its recursion pattern. For simplicity, we sometimes write $t$ instead of $\{t\}$ for singleton (multi)sets of terms, i.e., in the first rule, we write $\mathrm{f}_{0}(x) \stackrel{0}{\rightarrow} \mathrm{fib}(x)$ instead of $\mathrm{f}_{0}(x) \stackrel{0}{\rightarrow}\{\mathrm{fib}(x)\}$. Here, $\mathrm{f}_{0} \in \Sigma$ is the canonical start symbol, i.e., the entry point of the program:

$$
\begin{aligned}
\mathrm{f}_{0}(x) & \stackrel{0}{\rightarrow} \\
\mathrm{fib}(x), & \stackrel{1}{\rightarrow}\{\mathrm{fib}(x-1), \mathrm{fib}(x-2)\}[x>1], \\
\mathrm{fib}(x) \stackrel{1}{\rightarrow} \emptyset & {[x \leq 1] . }
\end{aligned}
$$

As the right-hand side of rule (2) consists of two terms, it is not tail-recursive. Note that the result of the fib program is not represented in the abstraction.

Using multisets as right-hand sides allows us to express that a function $f$ calls several other functions $f_{1}, \ldots, f_{n}$. Note that a rule of the form $f(\ldots) \rightarrow\left\{f_{1}(\ldots), \ldots, f_{n}(\ldots)\right\}[\varphi]$ is not equivalent to the $n$ rules $f(\ldots) \rightarrow f_{i}(\ldots)[\varphi]$ with $1 \leq i \leq n$ : While the former rule expresses that $f$ invokes all functions $f_{1}, \ldots, f_{n}$, the latter rules mean that $f$ non-deterministically invokes some

\footnotetext{
${ }^{2}$ Note that negations can be expressed by negating inequations directly, and disjunctions in programs can be expressed using several rules. We write " $s=t$ " as syntactic sugar for " $s \geq t \wedge s \leq t$ ".

${ }^{3}$ Note that rules with right-hand side $\emptyset$ can equivalently be transformed to rules with right-hand side "sink" where sink is a fresh function symbol of arity 0 . Thus, w.l.o.g., for tail-recursive programs, we assume that all rules have degree 1 .
} 


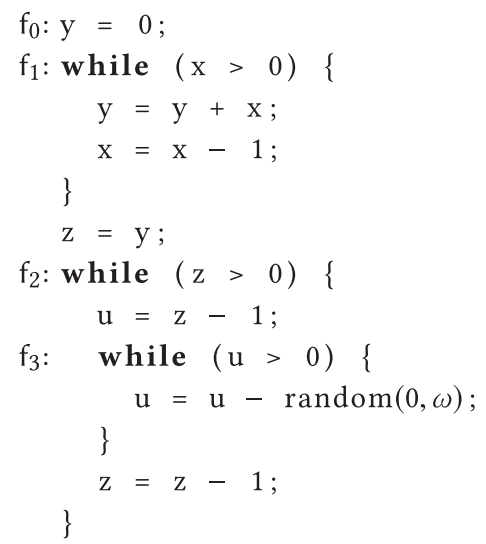

(a) Example Integer Program

$$
\begin{array}{lll}
\alpha_{0}: \mathrm{f}_{0}(x, y, z, u) & \stackrel{1}{\rightarrow} \mathrm{f}_{1}(x, 0, z, u) & \\
\alpha_{1}: \mathrm{f}_{1}(x, y, z, u) \stackrel{1}{\rightarrow} \mathrm{f}_{1}(x-1, y+x, z, u) & {[x>0]} \\
\alpha_{2}: \mathrm{f}_{1}(x, y, z, u) \stackrel{1}{\rightarrow} \mathrm{f}_{2}(x, y, y, u) & {[x \leq 0]} \\
\alpha_{3}: \mathrm{f}_{2}(x, y, z, u) \stackrel{1}{\rightarrow} \mathrm{f}_{3}(x, y, z, z-1) & {[z>0]} \\
\alpha_{4}: \mathrm{f}_{3}(x, y, z, u) \stackrel{1}{\rightarrow} \mathrm{f}_{3}(x, y, z, u-t v) & {[u>0 \wedge t v>0]} \\
\alpha_{5}: \mathrm{f}_{3}(x, y, z, u) \stackrel{1}{\rightarrow} \mathrm{f}_{2}(x, y, z-1, u) & {[u \leq 0]}
\end{array}
$$

Fig. 1. Different representations of integer programs.

random $(x, y)$ returns a random integer $t v$ with $x<t v<y$ and $\omega$ is the smallest infinite ordinal, i.e., we have $-\omega<n<\omega$ for all numbers $n \in \mathbb{Z}$. The following definition clarifies how to evaluate integer programs:

Definition 2.3 (Integer Transition Relation). A configuration is a multiset of terms of the form $f(\bar{n})$ where $f \in \Sigma$ and $\bar{n} \subset \mathbb{Z}$. The set of all configurations is denoted by $C$.

Let $\mathcal{P}$ be an integer program. For configurations $S, T \in C$, and $k \in \mathbb{R}, S$ evaluates to $T$ with cost $k\left(S \stackrel{k}{\rightarrow}_{\mathcal{P}} T\right)$ if there is an $s \in S$, a rule $\alpha$ of the form $f(\bar{x}) \stackrel{c}{\rightarrow} Q[\varphi] \in \mathcal{P}$, and an integer substitution $\sigma$ with $\mathcal{V}(\alpha) \subseteq \operatorname{dom}(\sigma)$ such that $f(\bar{x}) \sigma=s,{ }^{5} T=(S \backslash\{s\}) \cup Q \sigma,{ }^{6} \sigma \mid=\varphi$, and $c \sigma=k$.

For any integer program rule $\alpha$ and any integer substitution $\sigma$, let $\sigma \mid=\alpha$ denote that $\mathcal{V}(\alpha) \subseteq$ $\operatorname{dom}(\sigma)$ and $\sigma=\operatorname{guard}(\alpha)$.

We write $S \stackrel{k}{\rightarrow}$ $T$ instead of $S \stackrel{k}{\rightarrow} \mathcal{P} T$ if $\mathcal{P}$ is the singleton set containing $\alpha$. Moreover,

$$
S_{0} \stackrel{k \underset{P}{\rightarrow}}{\rightarrow} S_{m} \text { denotes that } S_{0} \stackrel{k_{1}}{\longrightarrow}{ }_{\mathcal{P}} \ldots \stackrel{k_{m}}{\longrightarrow} S_{m} \text { for } k=\sum_{i=1}^{m} k_{i} .
$$

If $m$ is irrelevant, we write $S_{0} \stackrel{k}{\rightarrow} \stackrel{*}{\rho} S_{m}$ if $m \geq 0$ and $S_{0} \stackrel{k}{\rightarrow} \underset{\mathcal{p}}{+} S_{m}$ if $m>0$. Finally, we sometimes omit the costs (of both rules and evaluations) if they are not important.

We say that a program is simplified if $\operatorname{root}(\alpha)=\mathrm{f}_{0}$ for all rules $\alpha$, i.e., if all left-hand sides of rules are constructed with the canonical start symbol $f_{0}$ that does not occur on right-hand sides. So any run of a simplified program that starts with a term of the form $\mathrm{f}_{0}(\bar{n})$ has at most length 1 .

Example 2.4 (Evaluation of Integer Programs). Using the rules from Figure 1(b), we have, e.g.,

$$
\mathrm{f}_{0}(3,2,1,0) \stackrel{1}{\rightarrow} \alpha_{0} \mathrm{f}_{1}(3,0,1,0) \stackrel{1}{\rightarrow} \alpha_{1} \mathrm{f}_{1}(2,3,1,0) \stackrel{1}{\rightarrow} \alpha_{1} \mathrm{f}_{1}(1,5,1,0) \stackrel{1}{\rightarrow}_{\alpha_{1}} \ldots
$$

For the rules $\mathcal{P}_{\text {fib }}=\{(1),(2),(3)\}$ from Example 2.1, we have

$$
\mathrm{f}_{0}(3) \stackrel{0}{\rightarrow} \mathcal{P}_{\text {fib }} \text { fib(3) } \stackrel{1}{\rightarrow}_{\mathcal{P}_{\text {fib }}}\{\mathrm{fib}(2), \mathrm{fib}(1)\} \stackrel{1}{\rightarrow} \mathcal{P}_{\text {fib }}\{\mathrm{fib}(1), \mathrm{fib}(0), \mathrm{fib}(1)\} \stackrel{3}{\rightarrow} \stackrel{3}{\text { fib }}_{\text {fib }} \emptyset,
$$

\footnotetext{
${ }^{5}$ Throughout this article, "=" means equality modulo arithmetic and we assume that ground arithmetic expressions and comparisons are evaluated exhaustively, so we have, e.g., $f(2)=f(3-1)$ and $3-1 \in \mathbb{Z}$.

${ }^{6}$ As usual, $S \backslash\{s\}$ means that the number of occurrences of $s$ in the multiset $S$ (if any) is reduced by 1 . We lift substitutions to (multi)sets of terms in the obvious way, i.e., $Q \sigma=\{q \sigma \mid q \in Q\}$.
} 
where $\{\mathrm{fib}(1)$, fib(0), fib(1) $\} \stackrel{3}{\rightarrow} \stackrel{3}{\mathcal{P}}_{\text {fib }} \emptyset$ abbreviates the three steps

$$
\{\mathrm{fib}(1), \mathrm{fib}(0), \mathrm{fib}(1)\} \stackrel{1}{\rightarrow} \mathcal{P}_{\mathrm{fib}}\{\mathrm{fib}(0), \mathrm{fib}(1)\} \stackrel{1}{\rightarrow}_{\mathcal{P}_{\mathrm{fib}}}\{\mathrm{fib}(1)\} \stackrel{1}{\rightarrow}_{\mathcal{P}_{\text {fib }}} \emptyset,
$$

whose combined cost is $1+1+1=3$.

According to our definition, integer programs may also contain rules like $f(x) \rightarrow f\left(\frac{x}{2}\right)$. While evaluations cannot yield non-integer values (e.g., we cannot evaluate $f(1)$ to $f\left(\frac{1}{2}\right)$, as $f\left(\frac{1}{2}\right)$ is not a configuration), our technique assumes that arithmetic expressions on right-hand sides of rules always map integers to integers. Hence, throughout this article, we restrict ourselves to well-formed integer programs.

Definition 2.5 (Well-formed Integer Program). An integer program rule $\alpha$ is well formed if $t_{i} \sigma \in \mathbb{Z}$ for each $f\left(t_{1}, \ldots, t_{k}\right) \in \operatorname{rhs}(\alpha)$, for each $1 \leq i \leq k$, and for each integer substitution $\sigma$ with $\sigma=\alpha$. An integer program is well formed if each of its rules is well formed.

Note that for a well-formed rule $\alpha$, we do not require $\operatorname{cost}(\alpha) \sigma \in \mathbb{Z}$ for integer substitutions $\sigma$ with $\sigma \mid=\alpha$.

To ensure that the analyzed program $\mathcal{P}_{0}$ is initially well formed, we just allow integer numbers, addition, subtraction, and multiplication in $\mathcal{P}_{0}{ }^{7}$ Our approach relies on several program transformations, i.e., the initial program $\mathcal{P}_{0}$ is transformed into other programs $\mathcal{P}_{1}, \mathcal{P}_{2}, \ldots$ that may contain further operations such as division and exponentiation. However, all our transformations preserve well-formedness.

To simplify the presentation, throughout this article, we assume that all function symbols in $\Sigma$ have the same arity. Otherwise, one can construct a variant of $\mathcal{P}$ where additional unused arguments are added to each function symbol whose arity is not maximal. Moreover, we assume that the left-hand sides of $\mathcal{P}$ only differ in their root symbols, i.e., the argument lists are equal (e.g., in Figure $1(\mathrm{~b})$, the variables on the left-hand sides are consistently named $x, y, z, u)$. Otherwise, one can rename variables accordingly without affecting the relation $\rightarrow_{\mathcal{p}}$. The variables $\bar{x}$ on the lefthand sides are called program variables and for any rule $\alpha$, all other variables $\mathcal{T} \mathcal{V}(\alpha)=\mathcal{V}(\alpha) \backslash \bar{x}$ are called temporary. These temporary variables are used to model non-deterministic program data. So in Figure 1(b), we have $\bar{x}=(x, y, z, u)$ and $t v \in \mathcal{T} \mathcal{V}\left(\alpha_{4}\right)$.

In Figure 1, the loop at $\mathrm{f}_{1}$ computes a value for $y$ that is quadratic in the original value of $x$. Thus, the loop at $f_{2}$ is executed quadratically often where in each iteration, the inner loop at $f_{3}$ may also be repeated quadratically often. Thus, the program's (worst-case) runtime is a polynomial of degree 4 in $x$. In contrast, the best-case runtime of the program is only quadratic in the original value of $x$, because then the inner loop at $\mathrm{f}_{3}$ would always set $u$ to a non-positive value immediately. The goal of our approach is to infer lower bounds for worst-case runtimes automatically.

To formalize the (worst-case) runtime complexity of an integer program, we define the derivation height of a configuration $S$ to be the cost of the most expensive evaluation starting with $S$. Here, for any non-empty set $M \subseteq \mathbb{R} \cup\{\omega\}$, sup $M$ is the least upper bound of $M$. In the following, let $\mathbb{R}_{\geq 0}=\{k \in \mathbb{R} \mid k \geq 0\}:$

Definition 2.6 (Derivation Height [44]). Let $\mathcal{P}$ be an integer program. Its derivation height function $\operatorname{dh}_{\mathcal{P}}: C \rightarrow \mathbb{R}_{\geq 0} \cup\{\omega\}$ is defined as $\operatorname{dh}_{\mathcal{P}}(S)=\sup \left\{k \in \mathbb{R} \mid S \stackrel{k^{*}}{\rightarrow} T\right.$ for some $\left.T \in C\right\}$.

Clearly, we always have $\operatorname{dh}_{\mathcal{P}}(S) \geq 0$, since $\stackrel{k^{*}}{\rightarrow}$ also permits evaluations with 0 steps. For the integer program $\mathcal{P}$ in Figure $1(\mathrm{~b})$, we obtain $\operatorname{dh}_{\mathcal{P}}\left(\mathrm{f}_{0}(0, y, z, u)\right)=2$ for all $y, z, u \in \mathbb{Z}$, since then

\footnotetext{
${ }^{7}$ One could also allow expressions with non-integer numbers like $\frac{1}{2} x^{2}+\frac{1}{2} x$ in the initial program, as long as every arithmetic expression in the program evaluates to an integer when instantiating its variables by integers. We will present a criterion to detect such expressions in Lemma 3.15.
} 
we can only apply the transitions $\alpha_{0}$ and $\alpha_{2}$ once. For all terms $\mathrm{f}_{0}(x, y, z, u)$ with $x>1, \alpha_{0}$ is executed once, then $\alpha_{1}$ is executed $x$ times. Afterwards, $y$ has the value $\frac{(x+1) \cdot x}{2}$. Now $\alpha_{2}$ is executed once and sets $z$ to the value $\frac{(x+1) \cdot x}{2}$. The outer loop at $\mathrm{f}_{2}$ is executed $z$ times, where in each iteration, the inner loop at $\mathrm{f}_{3}$ is executed $z-1$ times (in the worst case) and $z$ is decreased by 1 in $\alpha_{5}$. So overall, $\alpha_{3}$ and $\alpha_{5}$ are both executed $z$ times and $\alpha_{4}$ is executed $(z-1)+(z-2)+\cdots+1=$ $\frac{z \cdot(z-1)}{2}$ times. Hence, the worst-case runtime is $1+x+1+z+\frac{z \cdot(z-1)}{2}+z$, where $z=\frac{(x+1) \cdot x}{2}$, i.e., $\operatorname{dh}_{\mathcal{P}}\left(\mathrm{f}_{0}(x, y, z, u)\right)=\frac{1}{8} x^{4}+\frac{1}{4} x^{3}+\frac{7}{8} x^{2}+\frac{7}{4} x+2$. Our method will detect that the derivation height of $\mathrm{f}_{0}(x, y, z, u)$ is at least $\frac{1}{8} x^{4}+\frac{1}{4} x^{3}+\frac{7}{8} x^{2}+\frac{7}{4} x$. From this concrete lower bound, our approach will infer that the asymptotic runtime complexity of the program is in $\Omega\left(n^{4}\right)$ where $n$ is the size of the input, i.e., $n=|x|+|y|+|z|+|u|$. So the size of the input is measured by the sum of the absolute values of all program variables.

Note that the derivation height can be unbounded even if the integer program terminates.

Example 2.7 (Unbounded Costs without Non-termination). Consider the integer program $\mathcal{P}$ with the rule $\mathrm{f}_{0} \stackrel{t v}{\longrightarrow} \mathrm{f}$ where $t v$ is a temporary variable of the rule. By Definition 2.3, every integer substitution $\sigma$ with $t v \in \operatorname{dom}(\sigma)$ can be used to evaluate $\mathrm{f}_{0}$ to $\mathrm{f}$. Thus, for any $n \in \mathbb{N}$, we have $\mathrm{f}_{0} \stackrel{n}{\rightarrow} \mathrm{f}$ using an integer substitution $\sigma_{n}$ with $t v \sigma_{n}=n$. Therefore, we obtain $\operatorname{dh} \mathcal{P}\left(\mathrm{f}_{0}\right)=\omega$ although the rule has no recursive call.

Similarly, for the program $\mathcal{P}^{\prime}$ with the rules $\mathrm{f}_{0}(x) \stackrel{0}{\rightarrow} \mathrm{f}(t v)$ and $\mathrm{f}(x) \stackrel{1}{\rightarrow} \mathrm{f}(x-1)[x>0]$, we also have $\operatorname{dh}_{\mathcal{P}^{\prime}}\left(\mathrm{f}_{0}(1)\right)=\omega$, although every evaluation of the program is finite.

While $\mathrm{dh}_{\mathcal{P}}$ is defined on configurations, the complexity of a program is often defined as a function on $\mathbb{N}$, in particular when considering asymptotic complexity bounds. To bridge this gap, we use the common definition of complexity as a function of the size of the input. So the runtime complexity function $\operatorname{rc} \mathcal{P}(n)$ maps a natural number $n$ to the cost of the most expensive program run where the size of the input is bounded by $n$.

Definition 2.8 (Runtime Complexity). Let $\mathcal{P}$ be an integer program and let $k$ be the arity of $\mathrm{f}_{0}$. The runtime complexity function $\operatorname{rc} \mathcal{P}: \mathbb{N} \rightarrow \mathbb{R}_{\geq 0} \cup\{\omega\}$ of $\mathcal{P}$ is defined as

$$
\operatorname{rc} \mathcal{P}(n)=\sup \left\{\operatorname{dh}_{\mathcal{P}}\left(\mathrm{f}_{0}(\bar{n})\right)\left|\bar{n} \in \mathbb{Z}^{k},\right| \bar{n} \mid \leq n\right\},
$$

where for the vector $\bar{n}=\left(n_{1}, \ldots, n_{k}\right)$, we have $|\bar{n}|=\sum_{i=1}^{k}\left|n_{i}\right|$.

For the program $\mathcal{P}$ from Figure $1(\mathrm{~b})$, recall that the derivation height $\mathrm{dh}_{\mathcal{P}}\left(\mathrm{f}_{0}(x, y, z, u)\right)=\frac{1}{8} x^{4}+$ $\frac{1}{4} x^{3}+\frac{7}{8} x^{2}+\frac{7}{4} x+2$ solely depends on the value of the first argument $x$ of $\mathrm{f}_{0}$. As $n$ in Definition 2.8 is a bound on the sum of the absolute values of all arguments (i.e., $|x|+|y|+|z|+|u| \leq n$ ), setting $x=$ $n$ and $y=z=u=0$ maximizes $\operatorname{dh}_{\mathcal{P}}\left(\mathrm{f}_{0}(x, y, z, u)\right)$. Hence, we have $\operatorname{rc} \mathcal{P}(n)=\operatorname{dh}_{\mathcal{P}}\left(\mathrm{f}_{0}(n, 0,0,0)\right)=$ $\frac{1}{8} n^{4}+\frac{1}{4} n^{3}+\frac{7}{8} n^{2}+\frac{7}{4} n+2$.

Obviously, $\mathrm{dh}_{\mathcal{P}}$ and $\mathrm{rc} \mathcal{P}$ are not computable in general. Thus, our goal is to find a lower bound on the runtime complexity of a program $\mathcal{P}$ automatically that is as precise as possible (i.e., a lower bound that is, e.g., unbounded, exponential, or a polynomial of a degree as high as possible). So for the program in Figure 1(b), we would like to derive $\operatorname{rc} \mathcal{P}(n) \in \Omega\left(n^{4}\right)$, i.e., that the runtime complexity is asymptotically bounded from below by $n^{4}$. As usual, $f(n) \in \Omega(g(n))$ means that there is an $m>0$ and an $n_{0} \in \mathbb{N}$ such that $f(n) \geq m \cdot g(n)$ holds for all $n \geq n_{0}$. In our example, we also have $\operatorname{rc}_{\mathcal{P}}(n) \in O\left(n^{4}\right)$, i.e., $n^{4}$ is both an asymptotic lower and upper bound on the (worst-case) runtime complexity.

Note that according to Definition 2.8, $\operatorname{rc} \mathcal{P}(n)$ takes all runs into account that start with $\mathrm{f}_{0}(\bar{n})$ where the size $|\bar{n}|$ is $n$ or smaller (i.e., in the definition of $\operatorname{rc} \mathcal{P}(n)$, we use " $|\bar{n}| \leq n$ " instead of " $|\bar{n}|=$ $n$ "). This corresponds to the notion of "runtime complexity" used, e.g., for complexity analysis 
of term rewriting [43] or for complexity analysis of integer transition systems in the International Termination and Complexity Competition [37]. To see the difference between $\operatorname{rc} \mathcal{P}$ and the alternative definition

$$
\operatorname{rc}_{\mathcal{P}}^{\prime}(n)=\sup \left\{\operatorname{dh}_{\mathcal{P}}\left(\mathrm{f}_{0}(\bar{n})\right)\left|\bar{n} \in \mathbb{Z}^{k},\right| \bar{n} \mid=n\right\},
$$

consider a program $\mathcal{P}$ with the rule $\mathrm{f}_{0}(x) \stackrel{x}{\rightarrow} \mathrm{f}(x)[x \geq 0 \wedge x=2 \cdot t v]$. For non-negative numbers $n$, we have $\operatorname{rc}_{\mathcal{P}}^{\prime}(n)=\operatorname{dh} \mathcal{P}\left(\mathrm{f}_{0}(n)\right)=n$ and $\operatorname{rc} \mathcal{P}(n)=n$ if $n$ is even, but $\operatorname{rc}_{\mathcal{P}}^{\prime}(n)=\operatorname{dh} \mathcal{P}\left(\mathrm{f}_{0}(n)\right)=0$ and $\operatorname{rc} \mathcal{P}(n)=\operatorname{dh} \mathcal{P}\left(\mathrm{f}_{0}(n-1)\right)=n-1$ if $n$ is odd. As long as one is only interested in (asymptotic) upper bounds, the difference between $\operatorname{rc}_{\mathcal{P}}$ and $\operatorname{rc}_{\mathcal{P}}^{\prime}$ is negligible, since we have both $\operatorname{rc} \mathcal{P}(n) \in O(n)$ and $\operatorname{rc}_{\mathcal{P}}^{\prime}(n) \in O(n)$. (More precisely, for any program $\mathcal{P}$, we have $\operatorname{rc} \mathcal{P}(n) \in O(g(n))$ iff $\operatorname{rc}_{\mathcal{P}}^{\prime}(n) \in O(g(n))$ if $g$ is weakly monotonically increasing for large enough $n$.) But for (asymptotic) lower bounds, $\operatorname{rc} \mathcal{P}$ and $\mathrm{rc}_{\mathcal{P}}^{\prime}$ differ. For our example program, we have $\operatorname{rc} \mathcal{P}(n) \in \Omega(n)$, but $\operatorname{rc}_{\mathcal{P}}^{\prime}(n) \notin \Omega(n)$ (we only have $\left.\mathrm{rc}_{\mathcal{P}}^{\prime}(n) \in \Omega(1)\right)$. Recall that two of our main motivations for the inference of worst-case lower bounds are

(A) to deduce tight bounds in combination with existing techniques for the inference of worstcase upper bounds and

(B) to find denial-of-service vulnerabilities (or, more generally, performance bugs)

(see Section 1). The example above shows that to achieve (A), one should use our definition of $\operatorname{rc} \mathcal{P}$ instead of $\mathrm{rc}_{\mathcal{P}}^{\prime}{ }^{8}$ Regarding (B), note that $\operatorname{rc} \mathcal{P}(n) \in \Omega(g(n))$ means that for large enough $n$ one can always find inputs whose size is not greater than $n$, which lead to a runtime of at least $m \cdot g(n)$. Hence, our notion $\operatorname{rc} \mathcal{P}(n)$ can indeed be used to find families of program inputs that lead to runtimes of at least length $m \cdot g(n)$, for all large enough $n$. So if $g$ is unacceptably large (e.g., exponential or a high-degree polynomial), then such a family of program inputs witnesses a performance bug (which might, e.g., be exploited for denial-of-service attacks).

\section{SIMPLIFYING TAIL-RECURSIVE INTEGER PROGRAMS}

We now show how to transform any tail-recursive integer program $\mathcal{P}$ into a simplified program $\mathcal{P}^{\prime}$ such that the runtime complexity of $\mathcal{P}^{\prime}$ is smaller or equal to the runtime complexity of $\mathcal{P}$. Thus, any lower bound for $\operatorname{rc} \mathcal{P}$, is also a lower bound for $\operatorname{rc} \mathcal{P}$. In Section 4, we will extend our transformation to non-tail-recursive integer programs, before inferring asymptotic lower bounds for the runtime complexity of simplified programs in Section 5.

We first show in Section 3.1 how to under-estimate the number of possible loop iterations for simple loops $\alpha$ of the form $f(\bar{x}) \rightarrow f(\bar{x}) \mu[\varphi]$, where we define update $(\alpha)=\mu$ and require $\operatorname{dom}(\mu) \subseteq$ $\bar{x}$. So for instance, the rule

$$
\alpha_{1}: \mathrm{f}_{1}(x, y, z, u) \rightarrow \mathrm{f}_{1}(x-1, y+x, z, u) \quad[x>0]
$$

from Figure $1(\mathrm{~b})$ is a simple loop where update $\left(\alpha_{1}\right)$ is the substitution $\mu=\{x / x-1, y / y+x\}$. Based on the under-estimation of possible iterations, Section 3.2 presents our technique to accelerate simple loops. We introduce a technique to transform more complex loops into simple loops in Section 3.3.

\footnotetext{
${ }^{8}$ Nevertheless, almost all of our techniques would also work to infer a lower bound on $\mathrm{rc}_{\mathcal{P}}^{\prime}$ instead of $\mathrm{rc}_{\mathcal{P}}$. The only problem is in Section 5 where we search for an infinite family of inputs that satisfy the guard of the program. Here, it is not required that this family can represent inputs of size $n$ for all large enough $n$. So in our example, the technique of Section 5 would infer $\operatorname{rc}_{\mathcal{P}}(2 \cdot n) \in \Omega(n)$ (and indeed, we also have $\operatorname{rc}_{\mathcal{P}}^{\prime}(2 \cdot n) \in \Omega(n)$ ), but the family of inputs " $2 \cdot n$ " for all $n \geq 0$ does not represent all possible large enough numbers. For weakly monotonically increasing functions like $\mathrm{rc}_{\mathcal{P}}$, we present a technique in Section 5 (viz. Lemma 5.8) to transform a lower bound on $\operatorname{rc}_{\mathcal{P}}(2 \cdot n)$ into a lower bound on $\operatorname{rc}_{\mathcal{P}}(n)$, i.e., we show that $\mathrm{rc}_{\mathcal{P}}(2 \cdot n) \in \Omega(n)$ implies $\mathrm{rc}_{\mathcal{P}}(n) \in \Omega(n)$. But the technique of Lemma 5.8 is not applicable to $\mathrm{rc}_{\mathcal{P}}^{\prime}$, because $\mathrm{rc}_{\mathcal{P}}^{\prime}$ is not weakly monotonically increasing.
} 


\subsection{Under-estimating the Number of Iterations}

For a simple loop $\alpha$ of the form $f(\bar{x}) \rightarrow f(\bar{x}) \mu[\varphi]$, our goal is to infer an arithmetic expression $b$ such that for all integer substitutions $\sigma$ with $\sigma=\alpha$, the rule $\alpha$ can be executed at least $b \sigma$ times, i.e., there is an integer substitution $\sigma^{\prime}$ with $f(\bar{x}) \sigma \rightarrow_{\alpha}^{\lceil b \sigma\rceil} f(\bar{x}) \sigma^{\prime}$. Here, as usual, $\lceil x\rceil$ is the smallest integer $n$ with $n \geq x$.

To find such estimates, we use an adaptation of ranking functions $[6,9,13,56]$ that we call metering functions. In the following, we say that a quantifier-free formula $\varphi$ is valid if we have $\sigma \vDash \varphi$ for every integer substitution $\sigma$ with $\mathcal{V}(\varphi) \subseteq \operatorname{dom}(\sigma)$.

Definition 3.1 (Ranking Function). An arithmetic expression $b$ is a ranking function for a simple loop $\alpha$ with update $(\alpha)=\mu$ and $\mathcal{T} \mathcal{V}(\alpha)=\emptyset$ if the following conditions are valid:

$$
\begin{aligned}
& \operatorname{guard}(\alpha) \quad \Longrightarrow \quad b>0, \\
& \operatorname{guard}(\alpha) \quad \Longrightarrow \quad b \mu \leq b-1 .
\end{aligned}
$$

So for example, $x$ is a ranking function for the rule $\alpha_{1}$ in Figure 1(b), since both $x>0 \Longrightarrow x>0$ and $x>0 \Longrightarrow x-1 \leq x-1$ are clearly valid. If $b$ is a ranking function for a rule $\alpha$, then for any integer substitution $\sigma$ with $\mathcal{V}(\alpha) \subseteq \operatorname{dom}(\sigma), b \sigma$ over-estimates the number of possible iterations of the loop $\alpha$ : (7) ensures that $b \sigma$ decreases at least by 1 in each loop iteration (i.e., $b \mu \sigma \leq b \sigma-1$ holds whenever guard $(\alpha) \sigma$ is true), and Condition (6) requires that $b \sigma$ is positive whenever the loop can be executed.

Note that Definition 3.1 would be incorrect for the case $\mathcal{T} \mathcal{V}(\alpha) \neq \emptyset$. For example, consider the rule $\alpha: \mathrm{f}(x) \rightarrow \mathrm{f}(x+1)[x<t v]$. If we omitted the requirement $\mathcal{T} \mathcal{V}(\alpha)=\emptyset$, then $t v-x$ would be a ranking function for $\alpha$, since guard $(\alpha)$ implies both $t v-x>0$ and $t v-(x+1) \leq t v-x-1$. However, there are non-terminating evaluations like $\mathrm{f}(0) \rightarrow_{\alpha} \mathrm{f}(1) \rightarrow_{\alpha} \mathrm{f}(2) \rightarrow_{\alpha} \ldots$, since $t v$ can be instantiated differently in each evaluation step. Thus, $t v-x$ is not a correct over-estimation for the number of loop iterations.

To cover the case $\mathcal{T} \mathcal{V}(\alpha) \neq \emptyset$, Definition 3.1 would need to reflect that the values of temporary variables may change non-deterministically in every iteration. We chose the simple definition above, as it nicely exposes the analogy to our following novel concept of metering functions. In contrast to ranking functions, metering functions are under-estimates for the maximal number of iterations of a simple loop.

Definition 3.2 (Metering Function). We call an arithmetic expression $b$ a metering function for a simple loop $\alpha$ with update $(\alpha)=\mu$ if the following conditions are valid:

$$
\begin{array}{ll}
\neg \operatorname{guard}(\alpha) & \Longrightarrow \quad b \leq 0, \\
\operatorname{guard}(\alpha) & \Longrightarrow \quad b \mu \geq b-1 .
\end{array}
$$

Here, Condition (9) ensures that $b \sigma$ decreases at most by 1 in each loop iteration, and Condition (8) requires that $b \sigma$ is non-positive if the loop cannot be executed. Thus, the loop can be executed at least $b \sigma$ times (i.e., $b \sigma$ is an under-estimate).

In contrast to our definition of ranking functions, Definition 3.2 also covers the case $\mathcal{T} \mathcal{V}(\alpha) \neq \emptyset$. As we will show in Theorem 3.3, the reason is that a metering function $b$ for a simple loop $\alpha$ is a witness that $\alpha$ can be applied at least $b$ times for fixed values of $\alpha$ 's temporary variables. In particular, metering functions can also contain temporary variables to express that the number of loop iterations is unbounded, see Example 3.6.

As an example, for the loop $\alpha_{1}$ in Figure 1(b), $x$ is also a metering function. Condition (8) requires the validity of $\neg(x>0) \Longrightarrow x \leq 0$ and Condition (9) requires $x>0 \Longrightarrow x-1 \geq x-1$. While $x$ is a metering and a ranking function, $\frac{1}{2} x$ is a metering, but not a ranking function for $\alpha_{1}$. Similarly, 
$x^{2}$ is a ranking, but not a metering function for $\alpha_{1}$. Theorem 3.3 states that if $b$ is a metering function for a simple loop $\alpha$, then $\alpha$ can be executed at least $\lceil b \sigma\rceil$ times when starting the evaluation with lhs $(\alpha) \sigma$. Thus, if every rule has the constant cost 1 , then $\operatorname{dh}_{\{\alpha\}}(\operatorname{lhs}(\alpha) \sigma) \geq b \sigma$ holds for all integer substitutions $\sigma$ with $\mathcal{V}(\alpha) \cup \mathcal{V}(b) \subseteq \operatorname{dom}(\sigma)$. Recall that we have $\operatorname{rhs}(\alpha)=\operatorname{lhs}(\alpha) \mu$ for $\mu=$ update $(\alpha)$, since $\alpha$ is a simple loop. Hence, the evaluation has the form

$$
\operatorname{lhs}(\alpha) \sigma \rightarrow_{\alpha} \operatorname{rhs}(\alpha) \sigma=\operatorname{lhs}(\alpha) \mu \sigma \rightarrow_{\alpha} \operatorname{rhs}(\alpha) \mu \sigma=\operatorname{lhs}(\alpha) \mu^{2} \sigma \rightarrow_{\alpha} \ldots
$$

Here, for any $k \in \mathbb{N}, \mu^{k}$ stands for $k$ applications of $\mu$. So for example, $\mu^{3}$ stands for $\mu \circ \mu \circ \mu$ and $\mu^{0}$ is the identity substitution.

Theorem 3.3 (Metering Functions Under-Estimate Simple Loops). Let $b$ be a metering function for a well-formed simple loop $\alpha$ with $\mu=$ update $(\alpha)$. Then for all integer substitutions $\sigma$ with $\mathcal{V}(\alpha) \subseteq \operatorname{dom}(\sigma)$ and $\sigma \mid=b \geq 0$, there is the following evaluation of length $\lceil b \sigma\rceil$ :

$$
\operatorname{lhs}(\alpha) \sigma \rightarrow_{\alpha} \operatorname{lhs}(\alpha) \mu \sigma \rightarrow_{\alpha} \operatorname{lhs}(\alpha) \mu^{2} \sigma \rightarrow_{\alpha} \ldots \rightarrow_{\alpha} \operatorname{lhs}(\alpha) \mu^{\lceil b \sigma\rceil} \sigma,
$$

where $\mu^{k} \circ \sigma \mid=\operatorname{guard}(\alpha)$ for all $0 \leq k<b \sigma$.

Proof. For any integer substitution $\sigma$ with $\mathcal{V}(\alpha) \subseteq \operatorname{dom}(\sigma)$, let $m_{\sigma} \in \mathbb{N} \cup\{\omega\}$ be the length of the longest evaluation of the form $\operatorname{lhs}(\alpha) \sigma \rightarrow_{\alpha}^{m_{\sigma}} \operatorname{lhs}(\alpha) \mu^{m_{\sigma}} \sigma$ where $\mu^{k} \circ \sigma \mid=\operatorname{guard}(\alpha)$ for all $0 \leq k<m_{\sigma}$. So the loop $\alpha$ can be executed $m_{\sigma}$ times when starting with lhs $(\alpha) \sigma$. We prove that $m_{\sigma} \geq b \sigma$.

If $m_{\sigma}=\omega$, then the claim is trivial. For $m_{\sigma} \neq \omega$, we use induction on $m_{\sigma}$. In the base case $m_{\sigma}=0$, we have $\sigma \notin \operatorname{guard}(\alpha)$. Thus, Condition (8) implies $b \sigma \leq 0=m_{\sigma}$.

For the induction step $m_{\sigma} \geq 1$, we must have $\sigma \mid=\operatorname{guard}(\alpha)$, which implies:

$$
\begin{aligned}
b \mu \sigma & \geq b \sigma-1 \quad \text { by Condition (9) } \\
\operatorname{lhs}(\alpha) \sigma \rightarrow_{\alpha} \operatorname{lhs}(\alpha) \mu \sigma . &
\end{aligned}
$$

Due to (11), the longest evaluation $\operatorname{lhs}(\alpha) \sigma \rightarrow_{\alpha}^{m_{\sigma}} \operatorname{lhs}(\alpha) \mu^{m_{\sigma}} \sigma$ has the form

$$
\operatorname{lhs}(\alpha) \sigma \rightarrow_{\alpha} \operatorname{lhs}(\alpha) \mu \sigma \rightarrow_{\alpha}^{m_{\mu \circ \sigma}} \operatorname{lhs}(\alpha) \mu^{m_{\sigma}} \sigma,
$$

i.e., $m_{\sigma}=m_{\mu \circ \sigma}+1$. Since $\mathcal{V}(\alpha) \subseteq \operatorname{dom}(\mu \circ \sigma)$ and $\mu \circ \sigma$ is an integer substitution (as $\alpha$ is well formed), the induction hypothesis implies $m_{\mu \circ \sigma} \geq b \mu \sigma$. Hence, we have $m_{\sigma}=m_{\mu \circ \sigma}+1 \geq b \mu \sigma+$ $1 \geq b \sigma$ by (10).

Note that if one regards a single simple loop $f(\bar{x}) \rightarrow f(\bar{x}) \mu[\varphi]$ without any other $f$-rules that may lead to non-determinism, then the only remaining possible non-determinism is due to the temporary variables. So then the number of iterations of the loop in the worst and in the best case only depends on the instantiation of the temporary variables. Since the requirements (8) and (9) for the metering function must hold for all instantiations of the variables (i.e., also for all instantiations of the temporary variables), then a metering function is also a lower bound on the number of iterations of the loop in the best case. To exploit that we only need lower bounds on the worstcase runtime of the loop, in Section 3.2, we will present a technique that can instantiate temporary variables by suitable values that (hopefully) lead to long runtimes.

Our implementation builds upon a well-known transformation based on Farkas' Lemma $[13,56]$ to find linear metering functions. The basic idea is to search for coefficients of a linear template polynomial $b$ such that Conditions (8) and (9) hold for all possible instantiations of the variables $\mathcal{V}(\alpha)$. In addition to Conditions (8) and (9), we also require (6) to avoid trivial solutions like $b=0$. 
Here, the coefficients of $b$ are existentially quantified, while the variables from $\mathcal{V}(\alpha)$ are universally quantified. As in the approaches $[13,56]$, eliminating the universal quantifiers using Farkas' Lemma allows us to use standard SMT solvers to search for $b$ 's coefficients. ${ }^{9}$

If guard $(\alpha)$ contains constraints that are irrelevant for $\alpha$ 's termination (provided that guard $(\alpha)$ is satisfiable), then one can improve our approach. More precisely, if $\operatorname{guard}(\alpha)=\varphi \wedge \psi$, then $\psi$ is irrelevant for termination of the loop $\alpha$ if it always holds after executing the loop (given that it holds before the loop), i.e., if guard $(\alpha)$ implies $\psi \mu$. In this case, one can infer "conditional" metering functions of the form $\llbracket \psi \rrbracket \cdot b$. Here, $\llbracket \psi \rrbracket$ is the characteristic function of $\psi$, i.e., for any integer substitution $\sigma$ with $\mathcal{V}(\psi) \subseteq \operatorname{dom}(\sigma)$, we have $\llbracket \psi \rrbracket \sigma=1$ if $\sigma \mid=\psi$ and $\llbracket \psi \rrbracket \sigma=0$ otherwise. So for example, $\llbracket y+z=1 \rrbracket \sigma=1$ holds for an integer substitution $\sigma$ iff $y \sigma+z \sigma=1$.

Theorem 3.4 (Inferring Conditional Metering Functions). Let $\alpha$ be a simple loop such that update $(\alpha)=\mu$ and $\operatorname{guard}(\alpha)=\varphi \wedge \psi$ where $\operatorname{guard}(\alpha) \Longrightarrow \psi \mu$ is valid. If the conditions

$$
\begin{aligned}
& \neg \varphi \wedge \psi \quad \Longrightarrow \quad b \leq 0 \\
& \varphi \wedge \psi \quad \Longrightarrow \quad b \mu \geq b-1
\end{aligned}
$$

are valid, then $\llbracket \psi \rrbracket \cdot b$ is a metering function for $\alpha$.

Proof. If $\sigma \mid=\operatorname{guard}(\alpha)$, i.e., $\sigma \mid=\varphi \wedge \psi$, then we extend $\sigma$ arbitrarily to the variables in $\psi \mu$ that do not occur in $\varphi$ or $\psi$. Then, we have $\sigma=\psi \mu$ as $\operatorname{guard}(\alpha)$ implies $\psi \mu$. Thus, $\llbracket \psi \rrbracket \mu \sigma=1$ and $\llbracket \psi \rrbracket \sigma=1$. So by Condition (13), we obtain $(\llbracket \psi \rrbracket \cdot b) \mu \sigma=b \mu \sigma \geq b \sigma-1=(\llbracket \psi \rrbracket \cdot b) \sigma-1$, i.e., then $\llbracket \psi \rrbracket \cdot b$ satisfies Condition (9).

Now, we regard the case where $\sigma \mid=\neg \operatorname{guard}(\alpha)$. If $\sigma \mid=\neg \psi$, then $(\llbracket \psi \rrbracket \cdot b) \sigma=0$, i.e., then $\llbracket \psi \rrbracket \cdot b$ satisfies Condition (8). Otherwise, we have $\sigma \mid=\neg \varphi$ and $\sigma \mid=\psi$. Then Condition (12) implies ( $\llbracket \psi \rrbracket$. b) $\sigma=b \sigma \leq 0$, i.e., then $\llbracket \psi \rrbracket \cdot b$ also satisfies Condition (8).

While our implementation of Definition 3.2 was restricted to the search for linear metering functions $b$, with Theorem 3.4 our implementation can now also be used to obtain conditional metering functions of the form $\llbracket \psi \rrbracket \cdot b$ for linear arithmetic expressions $b$.

Compared to Definition 3.2, Theorem 3.4 weakens the conditions for metering functions: If $b$ is a metering function according to Conditions (8) and (9), then we can also prove that $\llbracket \psi \rrbracket \cdot b$ is a metering function via Theorem 3.4 (but the converse does not hold). The reason is that in Condition (8), we require $b \leq 0$ whenever $\neg(\varphi \wedge \psi)$ holds, whereas in Condition (12), we only require $b \leq 0$ whenever $\neg \varphi \wedge \psi$ holds.

Example 3.5 (Conditional Metering Function). To illustrate the use of conditional metering functions, consider the following rule $\alpha$ :

$$
\mathrm{f}(x, y, z) \rightarrow \mathrm{f}(x-y-z, y, z)[x>0 \wedge y+z=1] .
$$

Here, we can choose $\varphi$ to be $x>0$ and $\psi$ to be $y+z=1$, since $x>0 \wedge y+z=1$ implies $(y+z=$ 1) $\mu$ for $\alpha$ 's update $\mu=\{x / x-y-z\}$, i.e., it implies $y+z=1$. Hence, to infer the metering function $\llbracket y+z=1 \rrbracket \cdot x$, according to Theorem 3.4 it suffices to show that $\neg(x>0) \wedge y+z=1 \Longrightarrow x \leq 0$ and $x>0 \wedge y+z=1 \Longrightarrow x-y-z \geq x-1$ are valid. Using this metering function, our approach

\footnotetext{
${ }^{9}$ Since Farkas' Lemma is only applicable for linear constraints, for loops with non-linear arithmetic, our implementation uses simplifications to linearize the constraints that have to be satisfied for metering functions: We may substitute a nonlinear term by a fresh variable, provided that the variables of the non-linear term do not appear elsewhere (the reverse substitution is applied to the metering function afterwards), and we may omit irrelevant non-linear constraints or updates. For example, if the update of a variable $x$ that does not occur in the guard is non-linear, then we use a linear template polynomial $b$ without the variable $x$ for the metering function. But if such simplifications are not possible, then we fail when trying to infer metering functions for loops with non-linear arithmetic.
} 
can show that the rule can be applied at least linearly often. In contrast, without Theorem 3.4 our implementation would not be able to generate a useful metering function for this example, since it would only search for linear arithmetic expressions $b$ that satisfy Conditions (8) and (9). However, $x$ is not a metering function, since Condition (8) would not be satisfied (i.e., $\neg(x>0 \wedge y+z=$ 1) $\Longrightarrow x \leq 0$ is not valid).

In our earlier paper [29], we already sketched a related optimization, which is, however, weaker than Theorem 3.4. There the idea was to omit $\psi$ completely when searching for metering functions. So with this optimization, one would check the implications $\neg(x>0) \Longrightarrow x \leq 0$ and $x>0 \Longrightarrow$ $x-y-z \geq x-1$ to prove that $\llbracket y+z=1 \rrbracket \cdot x$ is a metering function for the loop of Example 3.5. As the second implication is not valid, this approach is not sufficient to handle Example 3.5. In contrast, Theorem 3.4 adds $\psi$ to the premise in Conditions (12) and (13), i.e., the approach of Theorem 3.4 for inferring conditional metering functions is strictly more powerful than the optimization from our paper [29].

Conditional metering functions are also particularly useful to integrate the handling of nonterminating rules in our approach.

Example 3.6 (Unbounded Loops). Let $\alpha$ be a simple loop whose update is $\mu$. If $\operatorname{guard}(\alpha) \Longrightarrow$ $\operatorname{guard}(\alpha) \mu$ is valid and hence the whole guard is irrelevant for $\alpha$ 's termination, then $\alpha$ does not terminate (provided that $\operatorname{guard}(\alpha)$ is satisfiable). In such cases, we can choose $\psi=\varphi$ in Theorem 3.4 and thus, Conditions (12) and (13) from Theorem 3.4 become

$$
\text { false } \Longrightarrow b \leq 0 \quad \text { and } \operatorname{guard}(\alpha) \Longrightarrow b \mu \geq b-1 \text {. }
$$

This is valid for a fresh temporary variable $b=t v$. Thus, for

$$
\mathcal{P}=\left\{\mathrm{f}_{0}(x, y) \stackrel{0}{\rightarrow} \mathrm{f}(x, y), \alpha\right\} \quad \text { where } \alpha \text { is the rule } \mathrm{f}(x, y) \stackrel{y}{\rightarrow} \mathrm{f}(x+1, y)[0<x],
$$

we obtain the metering function $\llbracket 0<x \rrbracket \cdot t v$. As $\operatorname{guard}(\alpha)=0<x$ is satisfiable, this indicates that the runtime of the program is unbounded, i.e., $\operatorname{dh}_{\mathcal{P}}(\mathrm{f}(x, y) \sigma) \geq t v \sigma$ and thus $\operatorname{dh}_{\mathcal{P}}(\mathrm{f}(x, y) \sigma)=\omega$ for all integer substitutions $\sigma$ with $\{x, y, t v\} \subseteq \operatorname{dom}(\sigma)$ and $0<x \sigma$.

Note that in this example, Theorem 3.4 succeeds when choosing $b$ to be $t v$ (i.e., $\llbracket 0<x \rrbracket \cdot t v$ is a metering function). In contrast, $t v$ is not a metering function, since Condition (8) does not hold (i.e., $\neg(0<x)$ does not imply $t v \leq 0)$. Thus, conditional metering functions allow us to handle terminating and non-terminating rules in a uniform way.

\subsection{Accelerating Simple Loops}

We now define sound processors that simplify integer programs. A sound processor is essentially a program transformation that preserves lower runtime bounds.

Definition 3.7 (Processor). A processor proc is a partial function that maps integer programs to integer programs. It is sound if $\operatorname{rc} \mathcal{P}(n) \geq \operatorname{rc}_{\operatorname{proc}(\mathcal{P})}(n)$ holds for all $n \in \mathbb{N}$ and all $\mathcal{P}$ where proc is defined.

In our framework, processors are applied repeatedly until the extraction of a concrete lower bound is straightforward. We first show how to accelerate a simple loop $\alpha$ to a rule that is equivalent to applying $\alpha$ multiple times (according to a metering function for $\alpha$ ). In Section 3.3, we will show that the resulting integer program can be simplified by chaining subsequent rules that may result in new simple loops. Moreover, we describe a simplification strategy that alternates these steps repeatedly. In this way, we eventually obtain a simplified program without loops that directly gives rise to a concrete lower bound. Section 3.2 only deals with simple loops, and in Section 3.3, we consider arbitrary tail-recursive rules $\alpha$ of the form $f(\bar{x}) \rightarrow g(\bar{t})[\varphi]$ with update $(\alpha)=\{\bar{x} / \bar{t}\}$ and $\operatorname{target}(\alpha)=g$. We extend our approach to arbitrary rules in Section 4. 
First, consider a simple loop $\alpha$ with update $(\alpha)=\mu$ and $\operatorname{cost}(\alpha)=c$. To accelerate $\alpha$, we compute its iterated update and cost, i.e., a substitution $\mu_{\text {it }}$ that is a closed form of $\mu^{t v}$ and an arithmetic expression $c_{\text {it }}$ that is an under-approximation of $\sum_{i=0}^{t v-1} c \mu^{i}$ for a fresh temporary variable $t v$, where $\mu^{i}$ again denotes the $i$-fold composition $\mu \circ \cdots \circ \mu$ of the substitution $\mu$. So $\mu_{\mathrm{it}}=\mu^{t v}$ and $c_{\text {it }} \leq \sum_{i=0}^{t v-1} c \mu^{i}$ must hold for all $t v>0$. If $\llbracket \psi \rrbracket \cdot b$ is a metering function for $\alpha$, then we add the accelerated rule

$$
\operatorname{lhs}(\alpha) \stackrel{c_{\text {itt }}}{\longrightarrow} \operatorname{lhs}(\alpha) \mu_{\mathrm{it}}[\operatorname{guard}(\alpha) \wedge \psi \wedge 0<t v<b+1]
$$

to the program. It summarizes $t v$ iterations of $\alpha$, where $t v$ is positive $\mathrm{e}^{10}$ and bounded by $\lceil b\rceil$. Note that $\mu_{\text {it }}$ and $c_{\text {it }}$ may also contain operations that are not allowed in the input program such as division and exponentiation (i.e., in this way, we can also infer non-polynomial bounds).

For the program variables $\bar{x}=\left(x_{1}, \ldots, x_{k}\right)$, the iterated update $\mu_{\text {it }}$ is computed by solving the recurrence equations $x^{(1)}=x \mu$ and $x^{(t v+1)}=x \mu\left\{x_{1} / x_{1}^{(t v)}, \ldots, x_{k} / x_{k}^{(t v)}\right\}$ for all $x \in \bar{x}$. So for the rule $\alpha_{1}$ from Figure 1(b), we get the recurrence equations $x^{(1)}=x-1, x^{\left(t v_{1}+1\right)}=x^{\left(t v_{1}\right)}-1, y^{(1)}=y+x$, and $y^{\left(t v_{1}+1\right)}=y^{\left(t v_{1}\right)}+x^{\left(t v_{1}\right)}$. Usually, the resulting equations can easily be solved using state-ofthe-art recurrence solvers [8, 41, 61]. In our example, we obtain the closed forms

$$
x \mu_{\text {it }}=x^{\left(t v_{1}\right)}=x-t v_{1} \quad \text { and } \quad y \mu_{\text {it }}=y^{\left(t v_{1}\right)}=y+t v_{1} \cdot x-\frac{1}{2} t v_{1}^{2}+\frac{1}{2} t v_{1} .
$$

While $y \mu_{\text {it }}$ contains rational coefficients, our approach ensures that $\mu_{\text {it }}$ always maps integers to integers. Thus, our technique to accelerate loops preserves well-formedness. We proceed similarly for the iterated cost of a rule, where we may under-approximate the solution of the recurrence equations $c^{(1)}=c$ and $c^{(t v+1)}=c^{(t v)}+c\left\{x_{1} / x_{1}^{(t v)}, \ldots, x_{k} / x_{k}^{(t v)}\right\}$. For the rule $\alpha_{1}$ in Figure 1 , we get $c^{(1)}=1$ and $c^{\left(t v_{1}+1\right)}=c^{\left(t v_{1}\right)}+1$, which leads to the closed form $c_{\mathrm{it}}=c^{\left(t v_{1}\right)}=t v_{1}$. Hence, when using $\alpha_{1}$ 's metering function $x$, it is accelerated to the following rule:

$$
\mathrm{f}_{1}(x, y, z, u) \stackrel{t v_{1}}{\longrightarrow} \mathrm{f}_{1}\left(x-t v_{1}, y+t v_{1} \cdot x-\frac{1}{2} t v_{1}^{2}+\frac{1}{2} t v_{1}, z, u\right) \quad\left[x>0 \wedge 0<t v_{1}<x+1\right] .
$$

Here, the guard can be simplified to $0<t v_{1}<x+1$. (We will perform such simplifications in all examples to ease readability.)

Theorem 3.8 (Loop AcCeleration). Let $\mathcal{P}$ be a well-formed integer program with the program variables $\bar{x}$, let $\alpha \in \mathcal{P}$ be a simple loop with update $(\alpha)=\mu$ and $\operatorname{cost}(\alpha)=c$, let tv be a fresh temporary variable, and let $\llbracket \psi \rrbracket \cdot b$ be a metering function for $\alpha$, where $b$ is an arithmetic expression. Moreover, for all $t v>0$, let $x \mu_{\mathrm{it}}=x \mu^{t v}$ be valid for all $x \in \bar{x}$, let $c_{\mathrm{it}} \leq \sum_{i=0}^{t v-1} c \mu^{i}$ be valid, and let

$$
\mathcal{P}^{\prime}=\mathcal{P} \cup\left\{\alpha_{\mathrm{it}}\right\} \quad \text { where } \alpha_{\mathrm{it}} \text { is the rule } \operatorname{lhs}(\alpha) \stackrel{c_{\mathrm{it}}}{\rightarrow} \operatorname{lhs}(\alpha) \mu_{\mathrm{it}}[\operatorname{guard}(\alpha) \wedge \psi \wedge 0<t v<b+1] .
$$

Then $\mathcal{P}^{\prime}$ is well formed and the processor that maps $\mathcal{P}$ to $\mathcal{P}^{\prime}$ is sound.

Proof. Let $\sigma$ be an integer substitution such that $\sigma \mid=\alpha_{\text {it }}$, i.e., we have

$$
\operatorname{lhs}(\alpha) \sigma \stackrel{c_{\mathrm{it}} \sigma}{\longrightarrow} \alpha_{\mathrm{it}} \operatorname{lhs}(\alpha) \mu_{\mathrm{it}} \sigma \text {. }
$$

Note that $\sigma \mid=0<t v<b+1$ implies $\sigma \mid=0<t v \leq\lceil b\rceil$ and we have $b \sigma=(\llbracket \psi \rrbracket \cdot b) \sigma$, because $\sigma \mid=$ $\operatorname{guard}\left(\alpha_{\mathrm{it}}\right)$ implies $\sigma \mid=\psi$ and thus, $\llbracket \psi \rrbracket \sigma=1$. Since $\sigma \mid=\operatorname{guard}\left(\alpha_{\mathrm{it}}\right)$ also implies $\sigma \mid=\llbracket \psi \rrbracket \cdot b \geq 0$ and $\llbracket \psi \rrbracket \cdot b$ is a metering function for $\alpha$, by Theorem 3.3 there is the following evaluation of length $t v \sigma$ :

$$
\operatorname{lhs}(\alpha) \sigma \rightarrow_{\alpha} \operatorname{lhs}(\alpha) \mu \sigma \rightarrow_{\alpha} \cdots \rightarrow_{\alpha} \operatorname{lhs}(\alpha) \mu^{t v \sigma} \sigma
$$

\footnotetext{
${ }^{10}$ The accelerated rule does not cover the case that $\alpha$ is not applied at all, i.e., it does not cover the case where $t v=0$. We excluded this case to ease the inference of the closed forms $\mu_{\text {it }}$ and $c_{\text {it }}$. To see this, consider a loop $\mathrm{f}(x) \rightarrow \mathrm{f}(0)$ with $x \mu=0$. Here, we would get $x \mu_{\text {it }}=0$ if $t v>0$ and $x \mu_{\text {it }}=x$ for $t v=0$. Hence, even in such simple examples it would be difficult to express the iterated update in closed form when considering the case $t v=0$ as well.
} 
where $\mu^{k} \circ \sigma \mid=\operatorname{guard}(\alpha)$ for all $0 \leq k<t v \sigma$. For that reason, the costs of the rule applications are $c \sigma, c \mu \sigma, \ldots, c \mu^{t v \sigma-1} \sigma$, i.e.,

$$
\operatorname{lhs}(\alpha) \sigma \stackrel{c \sigma}{\longrightarrow} \operatorname{lhs}(\alpha) \mu \sigma \stackrel{c \mu \sigma}{\longrightarrow} \ldots \stackrel{c \mu^{t v \sigma-1} \sigma}{\longrightarrow} \operatorname{lhs}(\alpha) \mu^{t v \sigma} \sigma .
$$

For soundness, it suffices to show that every evaluation step (15) with $\alpha_{\text {it }}$ can be simulated using a sequence of evaluation steps with $\alpha$ where the costs are at least the same. As $\alpha$ is well formed, this also implies well-formedness of $\alpha_{\mathrm{it}}$. By definition of $\mu_{\mathrm{it}}$ and $c_{\mathrm{it}}, x \mu_{\mathrm{it}}=x \mu^{t v}$ for all $x \in \bar{x}$ and $c_{\text {it }} \leq \sum_{i=0}^{t v-1} c \mu^{i}$ are valid. Thus, the evaluation (16) indeed simulates the evaluation step (15) with $\alpha_{\text {it }}$.

Note that Theorem 3.8 shows that when using conditional metering functions of the form $\llbracket \psi \rrbracket \cdot b$ (which we infer via Theorem 3.4) to accelerate loops, the characteristic function $\llbracket \psi \rrbracket$ is not needed in the accelerated rule. Instead, the condition $\psi$ is simply added to its guard. Thus, whenever the accelerated rule is applicable, then $\llbracket \psi \rrbracket \cdot b$ is equal to $b$ and hence, $\lceil b\rceil$ under-estimates the number of consecutive iterations of the original loop. Clearly, Theorem 3.8 is also applicable if the metering function is not conditional, i.e., if it is an ordinary arithmetic expression (by choosing $\psi=$ true).

The following example illustrates that the iterated update and cost may also contain nonpolynomial arithmetic, which may lead to exponential bounds.

Example 3.9 (Non-polynomial Arithmetic due to Loop Acceleration). Consider the program with the rule $\mathrm{f}_{0}(x, y) \stackrel{0}{\rightarrow} \mathrm{f}(x, y)$ and the simple loop $\mathrm{f}(x, y) \stackrel{y}{\rightarrow} \mathrm{f}(x-1,2 y)[x>0]$. Here, the update is $\mu=\{x / x-1, y / 2 y\}$ and hence, the resulting iterated update is $\mu_{\mathrm{it}}=\left\{x / x-t v, y / 2^{t v} \cdot y\right\}$. Moreover, the cost is $c=y$ and the iterated cost is $c_{\text {it }}=\sum_{i=0}^{t v-1} 2^{i} y=\left(2^{t v}-1\right) \cdot y$. Thus, both the iterated update and the iterated cost are exponential. Accelerating the simple loop via Theorem 3.8 with the metering function $x$ yields $\mathrm{f}(x, y) \stackrel{\left(2^{t v}-1\right) \cdot y}{\longrightarrow} \mathrm{f}\left(x-t v, 2^{t v} \cdot y\right)[0<t v<x+1]$, where we again simplified the guard $x>0 \wedge 0<t v<x+1$ to $0<t v<x+1$. Using this accelerated rule, our approach can infer an exponential lower bound for the program's runtime complexity.

Recall that the fresh variable $t v$ represents the number of loop iterations that are summarized by an accelerated rule. While $t v$ ranges over the integers, its upper bound $b+1$ can be rational, as the following example shows:

Example 3.10 (Non-integer Metering Functions). Theorem 3.8 also allows bounds that do not map to the integers. Consider the program

$$
\mathcal{P}=\left\{\mathrm{f}_{0}(x) \stackrel{0}{\rightarrow} \mathrm{f}(x), \alpha\right\} \quad \text { where } \alpha \text { is the rule } \mathrm{f}(x) \stackrel{1}{\rightarrow} \mathrm{f}(x-2)[0<x] .
$$

Clearly, $\frac{1}{2} x$ is a metering function for $\alpha$, as $\neg(0<x) \Longrightarrow \frac{1}{2} x \leq 0$ and $0<x \Longrightarrow \frac{1}{2}(x-2) \leq \frac{1}{2} x-$ 1 are valid. For $\mu=\{x / x-2\}$, we have $\mu_{\text {it }}=\mu^{t v}=\{x / x-2 t v\}$ and we choose $c_{\text {it }}=\sum_{i=0}^{t v-1} 1=t v$. Hence, accelerating $\alpha$ with the metering function $\frac{1}{2} x$ yields

$$
\mathrm{f}(x) \stackrel{t v}{\rightarrow} \mathrm{f}(x-2 t v)\left[0<t v<\frac{1}{2} x+1\right] .
$$

Note that $0<t v<\frac{1}{2} x+1$ implies $0<x$ as $t v$ ranges over $\mathbb{Z}$. Hence, $0<x$ can be omitted in the resulting guard.

If a (non-terminating) simple loop has the metering function $\llbracket \varphi \rrbracket \cdot t v$ where $t v$ is a fresh temporary variable, then the upper bound $b+1=t v+1$ on the number of summarized loop iterations can take arbitrary values.

Example 3.11 (Unbounded Loops Continued). In Example 3.6, $\llbracket 0<x \rrbracket \cdot t v$ is a metering function for $\alpha: \mathrm{f}(x, y) \stackrel{y}{\rightarrow} \mathrm{f}(x+1, y)[0<x]$. The resulting accelerated rule $\alpha_{\mathrm{it}}$ is

$$
\mathrm{f}(x, y) \stackrel{t v_{1} \cdot y}{\longrightarrow} \mathrm{f}\left(x+t v_{1}, y\right)\left[0<x \wedge 0<t v_{1}<t v+1\right]
$$


Since $t v$ does not have any upper bound, the value of $t v_{1}$ is not bounded by the values of the program variables $x$ and $y$. Thus, the condition of the rule could be replaced by $0<x \wedge 0<t v_{1}$, i.e., we obtain

$$
\mathrm{f}(x, y) \stackrel{t v_{1} \cdot y}{\longrightarrow} \mathrm{f}\left(x+t v_{1}, y\right)\left[0<x \wedge 0<t v_{1}\right] .
$$

After accelerating a simple loop $\alpha$ according to Theorem 3.8 using the metering function $\llbracket \psi \rrbracket \cdot b$, we eliminate the fresh variable $t v$ by instantiating it with $b$, provided that $b$ maps to $\mathbb{Z}$ (i.e., for every integer substitution $\sigma$ with $\sigma \mid=\alpha\{t v / b\}$, we have $b \sigma \in \mathbb{Z}$ ). The reason is that we want to keep the number of variables small for the sake of efficiency. However, this is just a heuristic that can also lead to worse results (e.g., if there is a non-terminating run where the original nonaccelerated loop must not be applied more than $b-1$ times after each other). If we cannot verify that $b \sigma \in \mathbb{Z}$ holds for every integer substitution $\sigma$ with $\sigma \mid=\alpha\{t v / b\}$, then we do not eliminate $t v$, but keep the inequation $0<t v<b+1$ in the accelerated rule.

We apply the following processor for the instantiation of temporary variables:

Theorem 3.12 (Instantiation). Let $\mathcal{P}$ be a well-formed integer program, let $\alpha \in \mathcal{P}$, let $t v \in$ $\mathcal{T} \mathcal{V}(\alpha)$, let $b$ be an arithmetic expression such that for every integer substitution $\sigma$ with $\sigma=\alpha\{t v / b\}$ we have $b \sigma \in \mathbb{Z}$, and let $\mathcal{P}^{\prime}=\mathcal{P} \cup\{\alpha\{t v / b\}\}$. Then $\mathcal{P}^{\prime}$ is well formed and the processor mapping $\mathcal{P}$ to $\mathcal{P}^{\prime}$ is sound.

Proof. To show the soundness of the processor, let $\sigma$ be an integer substitution with $\sigma=$ $\alpha\{t v / b\}$ and $b \sigma=m \in \mathbb{Z}$. Then let $\sigma^{\prime}$ be the integer substitution with $\operatorname{dom}\left(\sigma^{\prime}\right)=\operatorname{dom}(\sigma) \cup\{t v\}$, $\sigma^{\prime}(t v)=m$, and $\sigma^{\prime}(x)=\sigma(x)$ for all $x \in \operatorname{dom}(\sigma) \backslash\{t v\}$. Clearly, $\sigma \mid=\operatorname{guard}(\alpha\{t v / b\})$ iff $\sigma^{\prime} \mid=$ $\operatorname{guard}(\alpha)$ and moreover, $\operatorname{cost}(\alpha\{t v / b\}) \sigma=\operatorname{cost}(\alpha) \sigma^{\prime}$. Thus,

$$
\operatorname{lhs}(\alpha\{t v / b\}) \sigma \stackrel{k}{\rightarrow}_{\alpha\{t v / b\}} \operatorname{rhs}(\alpha\{t v / b\}) \sigma \quad \text { implies } \operatorname{lhs}(\alpha) \sigma^{\prime} \stackrel{k}{\rightarrow}_{\alpha} \operatorname{rhs}(\alpha) \sigma^{\prime} .
$$

This shows that every step with $\alpha\{t v / b\}$ can also be done with $\alpha$, because we have $\operatorname{lhs}(\alpha\{t v / b\}) \sigma=$ $\operatorname{lhs}(\alpha)\{t v / b\} \sigma=\operatorname{lhs}(\alpha)\{t v / m\} \sigma=\operatorname{lhs}(\alpha) \sigma^{\prime}$ (and $\operatorname{rhs}(\alpha\{t v / b\}) \sigma=\operatorname{rhs}(\alpha) \sigma^{\prime}$ can be derived analogously).

To show that $\mathcal{P}^{\prime}$ is well formed, recall that $\sigma \mid=\operatorname{guard}(\alpha\{t v / b\})$ iff $\sigma^{\prime} \mid=\operatorname{guard}(\alpha)$. If $\operatorname{rhs}(\alpha)$ contains $f\left(t_{1}, \ldots, t_{k}\right)$, then $t_{i} \sigma^{\prime} \in \mathbb{Z}$ holds for all $1 \leq i \leq k$ by well-formedness of $\mathcal{P}$. As $t_{i} \sigma^{\prime}=$ $t_{i}\{t v / b\} \sigma$, this implies well-formedness of $\mathcal{P}^{\prime}$.

Example 3.13 (Instantiation of Fresh Temporary Variables). For our example from Figure 1(b), accelerating $\alpha_{1}$ results in the rule (14). By instantiating its temporary variable $t v_{1}$ with the metering function $x$, the above processor yields

$$
\alpha_{\overline{1}}: \mathrm{f}_{1}(x, y, z, u) \stackrel{x}{\rightarrow} \mathrm{f}_{1}\left(0, y+\frac{1}{2} x^{2}+\frac{1}{2} x, z, u\right) \quad[x>0] .
$$

In our earlier paper [29, Theorem 10], we presented a processor that can extend the guard of a rule by arbitrary conjuncts. This processor could be used as an alternative to Theorem 3.12, because instead of instantiating $t v$, one could add the constraint " $t v=b$ " to the guard of $\alpha$. In practice, however, it is preferable to instantiate $t v$ to keep the number of variables as small as possible.

If we cannot apply Theorem 3.8 for a simple loop $\alpha$, because our implementation fails to solve the recurrence equations needed to compute the closed forms $\mu_{\text {it }}$ or $c_{\text {it }}$, or because it cannot find a useful metering function, then we can simplify $\alpha$ by eliminating temporary variables. To do so, we fix their values via Theorem 3.12. As we are interested in witnesses for maximal computations, we use a heuristic that sets $t v$ to $a$ for temporary variables $t v$ where the arithmetic expression $a$ is a minimal upper or a maximal lower bound on $t v$ 's values, i.e., guard $(\alpha)$ implies $t v \leq a$ but not $t v \leq a-1$, or guard $(\alpha)$ implies $t v \geq a$ but not $t v \geq a+1$. This elimination of temporary variables is repeated until we find constraints that allow us to apply loop acceleration. 
Example 3.14 (Instantiation of Other Temporary Variables). For the rule $\alpha_{4}$ from Figure 1(b), guard $\left(\alpha_{4}\right)$ contains the constraint $t v>0$. So guard $\left(\alpha_{4}\right)$ implies the bound $t v \geq 1$, since $t v$ must be instantiated by an integer. Hence, we instantiate the rule $\alpha_{4}$ by replacing $t v$ with 1 . Thus, the update $\{u / u-t v\}$ of the instantiated rule $\alpha_{4}^{\prime}$ becomes $\{u / u-1\}$. Hence, now $u$ is a metering function for $\alpha_{4}^{\prime}$ (whereas it was not a metering function for $\alpha_{4}$, as $u$ 's value could decrease by more than 1 in each application of $\alpha_{4}$ ). Thus, $\alpha_{4}^{\prime}$ can be accelerated similarly to $\alpha_{1}$, resulting in the rule

$$
\mathrm{f}_{3}(x, y, z, u) \stackrel{t v_{4}}{\longrightarrow} \mathrm{f}_{3}\left(x, y, z, u-t v_{4}\right)\left[0<t v_{4}<u+1\right] .
$$

Now the temporary variable $t v_{4}$ that results from loop acceleration can be eliminated by instantiating it with the metering function $u$. In this way, we obtain

$$
\alpha_{\overline{4}}: \mathrm{f}_{3}(x, y, z, u) \stackrel{u}{\rightarrow} \mathrm{f}_{3}(x, y, z, 0) \quad[u>0] .
$$

If $b$ is a polynomial, then we can use the following generalization of an observation on polynomials [18] to check the side condition of Theorem 3.12 that $b$ needs to map to $\mathbb{Z}$. Note that this check does not take the guard of the rule into account. So for the rule

$$
\mathrm{f}(x, y) \rightarrow \mathrm{f}(x-2, y-1)[x>0 \wedge x=2 \cdot y]
$$

with the metering function $\frac{x}{2}$ it would fail to recognize that $\sigma\left(\frac{x}{2}\right)$ is an integer for every model $\sigma$ of $x>0 \wedge x=2 \cdot y$.

Lemma 3.15 (Polynomials Mapping to $\mathbb{Z}$ ). Let $f: \mathbb{Z}^{k} \rightarrow \mathbb{R}$, where $f\left(x_{1}, \ldots, x_{k}\right)$ is a polynomial over the variables $x_{1}, \ldots, x_{k}$ with degrees $d_{1}, \ldots, d_{k}$ w.r.t. $x_{1}, \ldots, x_{k}$, respectively (i.e., for each $1 \leq i \leq k, f\left(x_{1}, \ldots, x_{k}\right)$ can be rearranged to the form $\sum_{j=0}^{d_{i}} p_{j} \cdot x_{i}^{j}$ where each $p_{j}$ is a polynomial over the variables $\left.x_{1}, \ldots, x_{i-1}, x_{i+1}, \ldots, x_{k}\right)$. If there are numbers $n_{1}, \ldots, n_{k} \in \mathbb{Z}$ such that $f\left(m_{1}, \ldots, m_{k}\right) \in \mathbb{Z}$ for all $m_{1}, \ldots, m_{k} \in \mathbb{Z}$ with $n_{i} \leq m_{i} \leq n_{i}+d_{i}+1$, then $\operatorname{img}(f) \subseteq \mathbb{Z}$, i.e., then we have $f\left(m_{1}, \ldots, m_{k}\right) \in \mathbb{Z}$ for all $m_{1}, \ldots, m_{k} \in \mathbb{Z}$.

Proof. We use induction on $d=\sum_{i=1}^{k} d_{i}$. If $d=0$, then $f$ is a constant and thus the claim is trivial. If $d>0$, then there exists a $1 \leq j \leq k$ with $d_{j}>0$. Then $g\left(x_{1}, \ldots, x_{k}\right)=f\left(x_{1}, \ldots, x_{j-1}, x_{j}+\right.$ $\left.1, x_{j+1}, \ldots, x_{k}\right)-f\left(x_{1}, \ldots, x_{k}\right)$ is a polynomial whose degree w.r.t. $x_{j}$ is $d_{j}-1$ and whose degree w.r.t. all $x_{i}$ with $i \neq j$ is at most $d_{i}$. To see this, note that all monomials that do not contain $x_{j}$ vanish in $g\left(x_{1}, \ldots, x_{k}\right)$. Thus, $g\left(x_{1}, \ldots, x_{k}\right)$ is a finite sum of expressions of the form $m \cdot\left(x_{j}+1\right)^{e}$. $p-m \cdot x_{j}^{e} \cdot p$ where $m \in \mathbb{R}, e \leq d_{j}$, and $p$ is a product of $x_{1}, \ldots, x_{j-1}, x_{j+1}, \ldots, x_{k}$ where each $x_{i}$, $i \neq j$, occurs at most $d_{i}$ times. We get:

$$
\begin{aligned}
& m \cdot\left(x_{j}+1\right)^{e} \cdot p-m \cdot x_{j}^{e} \cdot p \\
& =m \cdot\left(\sum_{i=0}^{e}\left(\begin{array}{l}
e \\
i
\end{array}\right) \cdot x_{j}^{i}\right) \cdot p-m \cdot x_{j}^{e} \cdot p \quad \text { by the Binomial theorem } \\
& =m \cdot\left(\left(\begin{array}{l}
e \\
e
\end{array}\right) \cdot x_{j}^{e}+q\right) \cdot p-m \cdot x_{j}^{e} \cdot p \quad \text { where } q \text { is a univariate polynomial over } x_{j} \\
& =m \cdot\left(x_{j}^{e}+q\right) \cdot p-m \cdot x_{j}^{e} \cdot p \quad \text { as }\left(\begin{array}{l}
e \\
e
\end{array}\right)=1 \\
& =m \cdot q \cdot p \text {. }
\end{aligned}
$$

Moreover, since $f\left(m_{1}, \ldots, m_{j}+1, \ldots, m_{k}\right) \in \mathbb{Z}$ and $f\left(m_{1}, \ldots, m_{j}, \ldots, m_{k}\right) \in \mathbb{Z}$ for all $m_{1}, \ldots, m_{k} \in \mathbb{Z}$ with $n_{j} \leq m_{j} \leq n_{j}+d_{j}$ and $n_{i} \leq m_{i} \leq n_{i}+d_{i}+1$ if $i \neq j$, we also have $g\left(m_{1}, \ldots, m_{k}\right) \in \mathbb{Z}$ for these $m_{1}, \ldots, m_{k}$. Thus, by the induction hypothesis, we obtain $\operatorname{img}(g) \subseteq \mathbb{Z}$. This means that we have $f\left(x_{1}, \ldots, x_{j-1}, x_{j}+1, x_{j+1}, \ldots, x_{k}\right)-f\left(x_{1}, \ldots, x_{k}\right) \in \mathbb{Z}$ for all $x_{1}, \ldots, x_{k} \in \mathbb{Z}$. Since this construction can be done for every $1 \leq j \leq k$ with $d_{j}>0$, we have $f\left(x_{1}, \ldots, x_{j-1}, x_{j}+1, x_{j+1}, \ldots, x_{k}\right)-f\left(x_{1}, \ldots, x_{k}\right) \in \mathbb{Z}$ for all $1 \leq j \leq k$ and all $x_{1}, \ldots, x_{k} \in \mathbb{Z}$. Thus, we also have $f\left(x_{1}, \ldots, x_{j-1}, x_{j}-1, x_{j+1}, \ldots, x_{k}\right)-f\left(x_{1}, \ldots, x_{k}\right) \in \mathbb{Z}$ for all $1 \leq j \leq k$ and 
all $x_{1}, \ldots, x_{k} \in \mathbb{Z}$. Since we also have $f\left(n_{1}, \ldots, n_{k}\right) \in \mathbb{Z}$ for some numbers $n_{1}, \ldots, n_{k} \in \mathbb{Z}$, this proves $\operatorname{img}(f) \subseteq \mathbb{Z}$.

So if $b$ is a polynomial, then it suffices to check if instantiating the variables in $b$ by finitely many integers always results in an integer. More precisely, if the polynomial $b$ contains the variables $x_{1}, \ldots, x_{k}$ of degrees $d_{1}, \ldots, d_{k}$, respectively, then we only check if the polynomial maps all arguments from $\left\{0, \ldots, d_{1}+1\right\} \times \cdots \times\left\{0, \ldots, d_{k}+1\right\}$ to integers. So, we choose $n_{i}=0$ for each $n_{i}$ from Lemma 3.15. This is not a restriction, because if there is some other $n_{i}^{\prime}$ such that $f\left(m_{1}, \ldots, m_{k}\right) \in \mathbb{Z}$ for all $m_{1}, \ldots, m_{k} \in \mathbb{Z}$ with $n_{i}^{\prime} \leq m_{i} \leq n_{i}^{\prime}+d_{i}+1$, then by Lemma 3.15 , we have $\operatorname{img}(f) \subseteq \mathbb{Z}$, which implies $f\left(m_{1}, \ldots, m_{k}\right) \in \mathbb{Z}$ for all $m_{1}, \ldots, m_{k} \in \mathbb{Z}$ with $n_{i}=0 \leq m_{i} \leq d_{i}+1=n_{i}+d_{i}+1$.

For instance, to check that the polynomial $\frac{1}{2} x^{2}+\frac{1}{2} x$ maps all $x \in \mathbb{Z}$ to integers, it suffices to check this for just $x \in\{0,1,2,3\}$. In contrast, for the polynomial $\frac{x}{2}$, we would check its value for $x \in\{0,1,2\}$ and determine that it does not always yield an integer.

Thus, one can implement Theorem 3.12 using Lemma 3.15 (but, of course, one may also incorporate further sufficient criteria). Similarly, as mentioned before, the criterion of Lemma 3.15 can also be used to check well-formedness of the integer program if we permit non-integer constants in the initial program.

To simplify the program, we delete the original rules after instantiating or accelerating them. If acceleration of a rule $\alpha$ still fails after eliminating all temporary variables by instantiating $\alpha$ repeatedly, then $\alpha$ is removed completely. So in the end, we just keep simple loops that have been accelerated. The following theorem shows that deleting rules is always sound:

Theorem 3.16 (Deletion). Let $\mathcal{P}$ be a well-formed integer program, let $\alpha \in \mathcal{P}$, and let $\mathcal{P}^{\prime}=\mathcal{P} \backslash$ $\{\alpha\}$. Then $\mathcal{P}^{\prime}$ is well formed and the processor mapping $\mathcal{P}$ to $\mathcal{P}^{\prime}$ is sound.

Proof. Since $\mathcal{P}$ is well formed, $\mathcal{P}^{\prime}$ is trivially well formed, too. The processor is sound, since every evaluation with $\mathcal{P} \backslash\{\alpha\}$ is also an evaluation with $\mathcal{P}$.

Example 3.17 (Examples 3.13 and 3.14 Continued). After accelerating and instantiating all simple loops in the program from Figure 1(b), we delete the original loops $\alpha_{1}$ and $\alpha_{4}$, resulting in the following integer program:

$$
\begin{array}{llll}
\alpha_{0}: \mathrm{f}_{0}(x, y, z, u) & \stackrel{1}{\rightarrow} & \mathrm{f}_{1}(x, 0, z, u) & \\
\alpha_{\overline{1}}: \mathrm{f}_{1}(x, y, z, u) & \stackrel{x}{\rightarrow} & \mathrm{f}_{1}\left(0, y+\frac{1}{2} x^{2}+\frac{1}{2} x, z, u\right) & {[x>0]} \\
\alpha_{2}: \mathrm{f}_{1}(x, y, z, u) & \stackrel{1}{\rightarrow} & \mathrm{f}_{2}(x, y, y, u) & {[x \leq 0]} \\
\alpha_{3}: \mathrm{f}_{2}(x, y, z, u) & \stackrel{1}{\rightarrow} & \mathrm{f}_{3}(x, y, z, z-1) & {[z>0]} \\
\alpha_{\overline{4}}: \mathrm{f}_{3}(x, y, z, u) & \stackrel{u}{\rightarrow} & \mathrm{f}_{3}(x, y, z, 0) & {[u>0]} \\
\alpha_{5}: \mathrm{f}_{3}(x, y, z, u) & \stackrel{1}{\rightarrow} & \mathrm{f}_{2}(x, y, z-1, u) & {[u \leq 0] .}
\end{array}
$$

\subsection{Chaining Rules}

After trying to accelerate all simple loops of a program, we can chain subsequent rules $\alpha_{1}, \alpha_{2}$ by adding a new rule $\alpha_{1.2}$ that represents their combination. Our notion of chaining corresponds to the standard notion of unfolding [16], adapted to our program model. Afterwards, the rules $\alpha_{1}$ and $\alpha_{2}$ can (but need not) be deleted with Theorem 3.16.

Theorem 3.18 (Chaining for Tail-Recursive Integer Programs). Let $\mathcal{P}$ be a well-formed tail-recursive integer program and let $\alpha_{1}, \alpha_{2} \in \mathcal{P}$ where

$$
\begin{array}{llllll}
\alpha_{1} & : & f_{1}(\bar{x}) & \stackrel{c_{1}}{\longrightarrow} & f_{2}(\bar{x}) \mu & {\left[\varphi_{1}\right] \text { and }} \\
\alpha_{2}: & f_{2}(\bar{x}) & \stackrel{c_{2}}{\longrightarrow} & t & {\left[\varphi_{2}\right] .}
\end{array}
$$


W.l.o.g., let $\mathcal{T} \mathcal{V}\left(\alpha_{1}\right) \cap \mathcal{T} \mathcal{V}\left(\alpha_{2}\right)=\emptyset$ (otherwise, the temporary variables in $\alpha_{2}$ can be renamed accordingly). Moreover, let $\alpha_{1.2}$ be the rule

$$
f_{1}(\bar{x}) \stackrel{c_{1}+c_{2} \mu}{\longrightarrow} t \mu\left[\varphi_{1} \wedge \varphi_{2} \mu\right]
$$

and let $\mathcal{P}^{\prime}=\mathcal{P} \cup\left\{\alpha_{1.2}\right\}$. Then $\mathcal{P}^{\prime}$ is well formed and the processor that maps $\mathcal{P}$ to $\mathcal{P}^{\prime}$ is sound.

Proof. We prove the more general Theorem 4.11 in Section 4.

One goal of chaining is to eliminate all accelerated simple loops. Therefore, after accelerating all simple loops, we chain all subsequent rules $\alpha^{\prime}, \alpha$ where $\alpha$ is a simple loop and $\alpha^{\prime}$ is not a simple loop. Afterwards, we delete $\alpha$. Moreover, once $\alpha^{\prime}$ has been chained with all subsequent simple loops, then we also remove $\alpha^{\prime}$, since its effect is now $\left(\right.$ mostly $\left.{ }^{11}\right)$ covered by the newly introduced chained rules.

Example 3.19 (Example 3.17 Continued). We continue the transformation of the program from Figure 1(b). Now, we chain $\alpha_{0}$ with the accelerated simple loop $\alpha_{\overline{1}}$, and we chain $\alpha_{3}$ with the accelerated simple loop $\alpha_{\overline{4}}$. This yields the following integer program:

$$
\begin{array}{lllll}
\alpha_{0 . \overline{1}}: & \mathrm{f}_{0}(x, y, z, u) & \stackrel{1+x}{\rightarrow} & \mathrm{f}_{1}\left(0, \frac{1}{2} x^{2}+\frac{1}{2} x, z, u\right) & {[x>0]} \\
\alpha_{2}: & \mathrm{f}_{1}(x, y, z, u) & \vec{\rightarrow} & \mathrm{f}_{2}(x, y, y, u) & {[x \leq 0]} \\
\alpha_{3 . \overline{4}}: & \mathrm{f}_{2}(x, y, z, u) & \vec{z} & \mathrm{f}_{3}(x, y, z, 0) & {[z>1]} \\
\alpha_{5}: & \mathrm{f}_{3}(x, y, z, u) & \overrightarrow{1} & \mathrm{f}_{2}(x, y, z-1, u) & {[u \leq 0] .}
\end{array}
$$

In rule $\alpha_{3 . \overline{4}}$, we simplified the guard $z>0 \wedge z-1>0$ to $z>1$.

Chaining also allows us to eliminate function symbols from the program by chaining all pairs of rules $\alpha^{\prime}$ and $\alpha$ where target $\left(\alpha^{\prime}\right)=\operatorname{root}(\alpha)$ and removing them afterwards. In this way, we can transform loops consisting of several transitions into simple loops. It is advantageous to eliminate symbols that are the target of just one single rule first. This heuristic avoids eliminating the entry points of loops, if possible.

Example 3.20 (Example 3.19 Continued). So for the program in Example 3.19, it would avoid chaining $\alpha_{5}$ and $\alpha_{3 . \overline{4}}$ where target $\left(\alpha_{5}\right)=\operatorname{root}\left(\alpha_{3 . \overline{4}}\right)=\mathrm{f}_{2}$, because $\mathrm{f}_{2}$ is also the target of the rule $\alpha_{2}$. In this way, we avoid constructing chained rules that correspond to a run from the "middle" of a loop to the "middle" of the next loop iteration.

Instead, we chain $\alpha_{0 . \overline{1}}$ and $\alpha_{2}$ as well as $\alpha_{3 . \overline{4}}$ and $\alpha_{5}$ to eliminate the function symbols $\mathrm{f}_{1}$ and $\mathrm{f}_{3}$. This leads to the following program:

$$
\begin{array}{lllll}
\alpha_{0 . \overline{1} .2}: & \mathrm{f}_{0}(x, y, z, u) & \stackrel{2+x}{\longrightarrow} & \mathrm{f}_{2}\left(0, \frac{1}{2} x^{2}+\frac{1}{2} x, \frac{1}{2} x^{2}+\frac{1}{2} x, u\right) & {[x>0]} \\
\alpha_{3 . \overline{4} .5}: & \mathrm{f}_{2}(x, y, z, u) & \stackrel{1+z}{\longrightarrow} & \mathrm{f}_{2}(x, y, z-1,0) & {[z>1] .}
\end{array}
$$

Our overall approach for program simplification is shown in Algorithm 1. It transforms any tail-recursive integer program into a simplified program. Section 5 will show how to analyze the (asymptotic) complexity of simplified programs. Of course, other strategies for the application of the processors would be possible, too. Recall that the application of Acceleration, Instantiation, and Chaining always generates new rules (so, e.g., in Step 2.2.1, instantiating $\bar{\alpha}$ generates a new rule $\widetilde{\alpha}$ and the original non-instantiated rule $\bar{\alpha}$ is deleted in Step 2.2.2). The set $S$ in the Steps $3-5$ is needed to handle function symbols $f$ with multiple simple loops. The reason is that each rule $\alpha^{\prime}$ with $\operatorname{target}\left(\alpha^{\prime}\right)=f$ should be chained with each of $f^{\prime}$ 's simple loops before removing $\alpha^{\prime}$.

\footnotetext{
${ }^{11}$ Since accelerated rules $\alpha_{\mathrm{it}}$ do not cover the case where $\alpha$ is not executed at all (see Footnote 10), chaining $\alpha^{\prime}$ with $\alpha_{\mathrm{it}}$ and deleting these rules afterwards does not cover those original evaluations where $\alpha^{\prime}$ was not followed by any subsequent application of $\alpha$. However, since we are only interested in witnesses for maximal evaluations, this does not affect the soundness of our approach.
} 


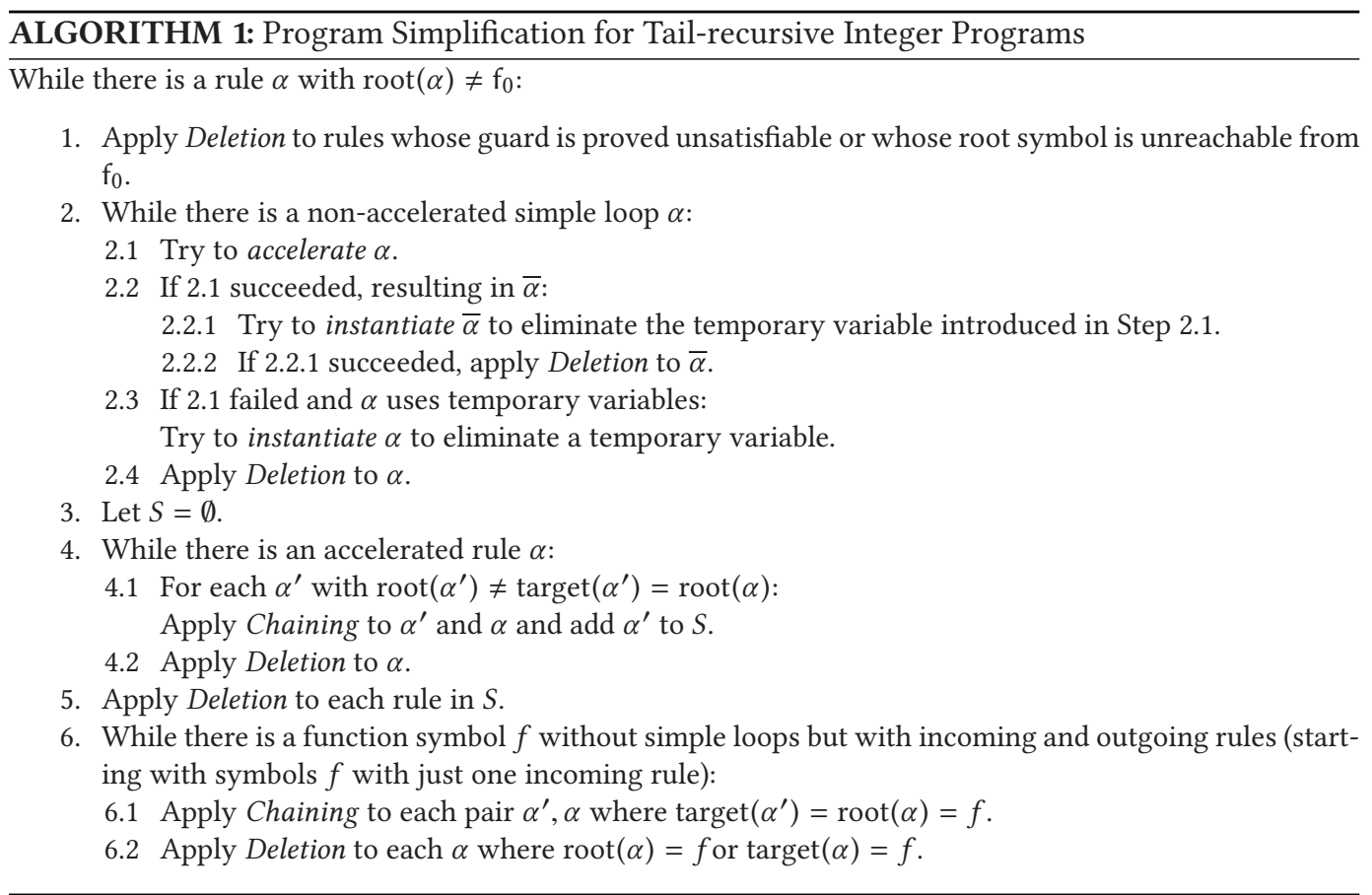

Algorithm 1 terminates: The loop in Step 2 terminates, since each iteration either decreases the number of temporary variables in $\alpha$ or reduces the number of non-accelerated simple loops. In Step 4, the number of accelerated rules is decreasing and for the loop in Step 6, the number of function symbols decreases. The overall loop of Algorithm 1 terminates as it reduces the number of function symbols. The reason is that the program does not have simple loops anymore when the algorithm reaches Step 6 (as simple loops where acceleration fails are deleted in Step 2.4 and accelerated rules are eliminated in Step 4). Thus, at this point there is either a function symbol $f$ that can be eliminated or the program does not have a path of length 2, i.e., all rules have the root $f_{0}$.

Example 3.21 (Example 3.20 Continued). According to Algorithm 1, in our example, we go back to Step 1 and 2 and apply Loop Acceleration to the rule $\alpha_{3 . \overline{4} .5}$. This rule has the metering function $z-1$ and its iterated update sets $u$ to 0 and $z$ to $z-t v$ for a fresh temporary variable $t v$. To compute $\alpha_{3 . \overline{4} .5}$ 's iterated cost, we have to find an under-approximation for the solution of the recurrence equations $c^{(1)}=1+z$ and $c^{(t v+1)}=c^{(t v)}+1+z^{(t v)}$. After computing the closed form $z-t v$ of $z^{(t v)}$, the second equation simplifies to $c^{(t v+1)}=c^{(t v)}+1+z-t v$, which results in the closed form $c_{\text {it }}=$ $c^{(t v)}=t v \cdot z-\frac{1}{2} t v^{2}+\frac{3}{2} t v$. Thus, we obtain the accelerated rule

$$
\mathrm{f}_{2}(x, y, z, u) \stackrel{t v \cdot z-\frac{1}{2} t v^{2}+\frac{3}{2} t v}{\longrightarrow} \mathrm{f}_{2}(x, y, z-t v, 0)[0<t v<z] .
$$

By instantiating $t v$ with $z-1$ in Step 2.2.1 and removing $\alpha_{3 . \overline{4} .5}$ in Step 2.4, we obtain the following program:

$$
\begin{aligned}
& \alpha_{0 . \overline{1} .2}: \quad \mathrm{f}_{0}(x, y, z, u) \quad \stackrel{2+x}{\longrightarrow} \quad \mathrm{f}_{2}\left(0, \frac{1}{2} x^{2}+\frac{1}{2} x, \frac{1}{2} x^{2}+\frac{1}{2} x, u\right) \quad[x>0] \\
& \alpha \overline{3 . \overline{4} .5}: \mathrm{f}_{2}(x, y, z, u) \stackrel{\frac{1}{2} z^{2}+\frac{3}{2} z-2}{\longrightarrow} \mathrm{f}_{2}(x, y, 1,0) \quad[z>1] .
\end{aligned}
$$


A final chaining step and deletion of $\alpha_{0 . \overline{1} .2}$ and $\alpha_{3 . \overline{4} .5}$ yields the simplified program with the following single rule:

$$
\alpha_{0 . \overline{1} \cdot 2 \cdot \overline{3 . \overline{4} .5}}: \mathrm{f}_{0}(x, y, z, u) \stackrel{\frac{1}{8} x^{4}+\frac{1}{4} x^{3}+\frac{7}{8} x^{2}+\frac{7}{4} x}{\longrightarrow} \mathrm{f}_{2}\left(0, \frac{1}{2} x^{2}+\frac{1}{2} x, 1,0\right)\left[\frac{1}{2} x^{2}+\frac{1}{2} x>1\right] .
$$

\section{SIMPLIFYING ARBITRARY RECURSIVE INTEGER PROGRAMS}

So far, we only considered tail-recursive programs, i.e., programs where all rules have the form $f(\bar{x}) \rightarrow g(\bar{t})[\varphi]$. We now extend our technique to non-tail-recursive programs, i.e., we now also consider rules where the right-hand side is a multiset of several terms.

Theorems 3.12 and 3.16 are trivially applicable to non-tail-recursive programs as well, i.e., we can still instantiate temporary variables and we can still delete rules. However, Theorems 3.8 and 3.18 have to be adapted. In Section 4.1, we extend our notion of metering functions to (nontail-)recursive rules to adapt Theorem 3.8. Similar to the case of tail-recursive programs where we started with considering simple loops, we first focus on simple recursions, i.e., rules $f(\bar{x}) \stackrel{c}{\rightarrow} T[\varphi]$ whose degree $|T|$ is greater than 1 and where all terms in $T$ have the root symbol $f .{ }^{12}$ Our extended notion of metering functions then allows us to accelerate simple recursions in Section 4.2. Afterwards, in Section 4.3, we show how to transform more complex recursions into simple recursions or simple loops via Chaining and Partial Deletion, a new technique that is specific to non-tailrecursive integer programs. Based on these techniques, we extend Algorithm 1 to a procedure that transforms any integer program into a simplified program.

\subsection{Under-estimating the Depth of Recursions}

To understand the idea of metering functions for simple recursions, note that repeatedly applying a simple recursive rule $f(\bar{x}) \stackrel{c}{\rightarrow} T[\varphi]$ essentially yields an evaluation tree of terms where each inner node has $|T|$ successors. While metering functions for simple loops under-estimate the length of evaluations, metering functions for simple recursions under-estimate the height up to which such evaluation trees are complete. Hence, if $b$ is a metering function for a simple recursion $\alpha$ of degree $d$, then the maximal number of consecutive applications of $\alpha$ is in $\Omega\left(d^{b}\right)$.

Definition 4.1 (Metering Function for Simple Recursions). Let $\alpha$ be a rule of the form

$$
f(\bar{x}) \stackrel{c}{\rightarrow} T[\varphi]
$$

such that $T$ contains no function symbol from $\Sigma$ except $f$. We call an arithmetic expression $b$ a metering function for $\alpha$ if the following conditions are valid:

$$
\begin{array}{ll}
\neg \operatorname{guard}(\alpha) & \Longrightarrow \quad b \leq 0, \\
\operatorname{guard}(\alpha) & \Longrightarrow \quad b\{\bar{x} / \bar{t}\} \geq b-1 \text { for all } f(\bar{t}) \in T .
\end{array}
$$

Definition 4.1 is a generalization of Definition 3.2, i.e., if $\alpha$ has degree 1, then Definition 4.1 and Definition 3.2 coincide. Moreover, note that conditional metering functions of the form $\llbracket \psi \rrbracket \cdot b$ for simple recursions can be inferred analogously to conditional metering functions for simple loops (see Theorem 3.4): If guard $(\alpha)$ is $\varphi \wedge \psi$ and guard $(\alpha)$ implies $\psi\{\bar{x} / \bar{t}\}$ for all $f(\bar{t}) \in T$, then it suffices to check $\neg \varphi \wedge \psi \Longrightarrow b \leq 0$ and $\varphi \wedge \psi \Longrightarrow b\{\bar{x} / \bar{t}\} \geq b-1$ for all $f(\bar{t}) \in T$ to prove that $\llbracket \psi \rrbracket \cdot b$ is a metering function for $\alpha$.

Example 4.2 (Metering Function for Fibonacci). According to Definition 4.1, $\frac{1}{2} x-1$ is a metering function for the recursive Fibonacci rule (2) from Example 2.1. It satisfies Condition (20), as we

\footnotetext{
${ }^{12}$ The reason for excluding the case $|T|=1$ is that, in this way, simple recursions give rise to exponential lower bounds, i.e., we can formulate the corresponding Theorem 4.3, which does not hold if $|T|=1$.
} 
have $\neg(x>1) \Longrightarrow \frac{1}{2} x-1 \leq 0$. The recursive call fib $(x-1)$ satisfies Condition (21), since we have

$$
x>1 \Longrightarrow \frac{1}{2}(x-1)-1=\frac{1}{2} x-\frac{3}{2} \geq \frac{1}{2} x-2 .
$$

Finally, the recursive call fib $(x-2)$ also satisfies Condition (21), as we have

$$
x>1 \Longrightarrow \frac{1}{2}(x-2)-1=\frac{1}{2} x-2 \geq \frac{1}{2} x-2 .
$$

For a simple loop $\alpha$, we directly use its metering function as a lower bound for the number of consecutive applications of $\alpha$. But for simple recursions, we can infer a lower bound that is higher than its metering function. The reason is that when computing a metering function $b$ for a simple recursion $f(\bar{x}) \rightarrow\left\{f\left(\overline{t_{1}}\right), \ldots, f\left(\overline{t_{d}}\right)\right\}[\varphi]$, we proceed as if we had $d$ separate tail-recursive rules $f(\bar{x}) \rightarrow f\left(\overline{t_{1}}\right)[\varphi], \ldots, f(\bar{x}) \rightarrow f\left(\overline{t_{d}}\right)[\varphi]$. However, as the original rule initiates $d$ new evaluations in each step, we obtain a lower bound on the length of evaluations that is exponential in $b$. In other words, since the metering function $b$ under-estimates the height of complete evaluation trees (where every non-leaf node has $d$ children), the number of edges of the tree is in $\Omega\left(d^{b}\right)$.

Theorem 4.3 (Metering Functions Under-estimate Simple Recursions). Let $b$ be a metering function for a well-formed simple recursion $\alpha$ with degree $d$. Then for all integer substitutions $\sigma$ with $\mathcal{V}(\alpha) \subseteq \operatorname{dom}(\sigma)$, there is an evaluation $\operatorname{lhs}(\alpha) \sigma \rightarrow_{\alpha}^{m} S$ for some configuration $S$ with $m \geq \frac{d^{b \sigma}-1}{d-1}$.

Proof. First note that we have $d>1$, since $\alpha$ is a simple recursion and hence $\frac{d^{b \sigma}-1}{d-1}$ is well defined. As in the proof of Theorem 3.3, let $m_{\sigma} \in \mathbb{N} \cup\{\omega\}$ be the length of the longest evaluation that starts with $\operatorname{lhs}(\alpha) \sigma$ and only applies the rule $\alpha$ repeatedly. We prove that $m_{\sigma} \geq \frac{d^{b \sigma}-1}{d-1}$.

The case $m_{\sigma}=\omega$ is trivial. For $m_{\sigma} \neq \omega$, we use induction on $m_{\sigma}$. In the base case $m_{\sigma}=0$, we have $\sigma \vDash \operatorname{guard}(\alpha)$ and thus, Condition (20) implies $b \sigma \leq 0$. Hence, $\frac{d^{b \sigma}-1}{d-1} \leq 0=m_{\sigma}$.

For the induction step $m_{\sigma} \geq 1$, we must have $\sigma \mid=\operatorname{guard}(\alpha)$. Let $\operatorname{lhs}(\alpha)=f(\bar{x})$. Then, we obtain

$$
\begin{aligned}
b\{\bar{x} / \bar{t}\} \sigma \geq b \sigma-1 & \text { for all } f(\bar{t}) \in \operatorname{rhs}(\alpha), \quad \text { by Condition (21) } \\
\operatorname{lhs}(\alpha) \sigma=f(\bar{x}) \sigma & { }_{\alpha} \operatorname{rhs}(\alpha) \sigma .
\end{aligned}
$$

Let $\operatorname{rhs}(\alpha)=\left\{f\left(\overline{t_{1}}\right), \ldots, f\left(\overline{t_{d}}\right)\right\}$. Then due to the evaluation step (23), the longest evaluation $\operatorname{lhs}(\alpha) \sigma \rightarrow_{\alpha}^{m_{\sigma}} S$ has the form

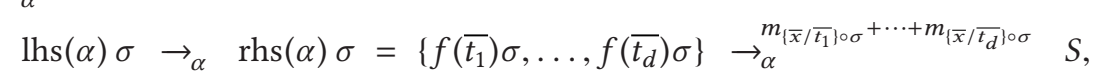

i.e., $m_{\sigma}=m_{\left\{\bar{x} / \overline{t_{1}}\right\} \circ \sigma}+\cdots+m_{\left\{\bar{x} / \overline{t_{d}}\right\} \circ \sigma}+1$. Since $\mathcal{V}(\alpha) \subseteq \operatorname{dom}\left(\left\{\bar{x} / \overline{t_{i}}\right\} \circ \sigma\right)$ and $\left\{\bar{x} / \overline{t_{i}}\right\} \circ \sigma$ is an integer substitution (since $\alpha$ is well formed), the induction hypothesis implies $m_{\left\{\bar{x} / \overline{t_{i}}\right\} \circ \sigma} \geq \frac{d^{b}\left\langle\bar{x} / \overline{t_{i}}\right\rangle \sigma-1}{d-1} \geq$ $\frac{d^{b \sigma-1}-1}{d-1}$ by Inequality (22) for all $1 \leq i \leq d$. Hence, we have $m_{\sigma}=m_{\left\{\bar{x} / \overline{t_{1}}\right\} \circ \sigma}+\cdots+m_{\left\{\bar{x} / \overline{t_{d}}\right\} \circ \sigma}+1 \geq$ $d \cdot \frac{d^{b \sigma-1}-1}{d-1}+1=\frac{d^{b \sigma}-d}{d-1}+1=\frac{d^{b \sigma}-1}{d-1}$.

Example 4.4 (Under-Estimating Fibonacci). Since $\frac{1}{2} x-1$ is a metering function for the recursive Fibonacci rule (2), the term $\operatorname{fib}(x) \sigma$ starts an evaluation of at least length $2^{\frac{1}{2} \cdot x \sigma-1}-1$ for each integer substitution $\sigma$ with $x \in \operatorname{dom}(\sigma)$.

\subsection{Accelerating Simple Recursions}

Using Definition 4.1 and Theorem 4.3, we can now accelerate simple recursions. In contrast to the acceleration of simple loops in Theorem 3.8, here, we disregard the result of the accelerated rule and replace its result with $\emptyset$. The reason is that otherwise the degree of the accelerated rule (i.e., the number of elements in its right-hand side) would depend on the instantiation of the variable $t v$ that represents the height of the evaluation tree. This cannot be expressed in our program model. Moreover, we cannot compute iterated updates, as a simple recursion $\alpha$ has several updates ACM Transactions on Programming Languages and Systems, Vol. 42, No. 3, Article 13. Publication date: October 2020. 
that may be applied in arbitrary order when $\alpha$ is applied repeatedly. Since computing the iterated cost would require the iterated updates, we do not compute the iterated cost anymore, but simply under-estimate each evaluation step with cost 1 . This suffices to infer a lower bound for the cost of the accelerated rule that is exponential in the metering function.

Theorem 4.5 (Recursion Acceleration). Let $\mathcal{P}$ be a well-formed integer program, let $\alpha \in \mathcal{P}$ be a simple recursion of degree $d$ such that

$$
\operatorname{guard}(\alpha) \quad \Longrightarrow \operatorname{cost}(\alpha) \geq 1
$$

is valid, ${ }^{13}$ and let $\llbracket \psi \rrbracket \cdot b$ be a metering function for $\alpha$. Moreover, let $\alpha^{\prime}$ be the rule

$$
\operatorname{lhs}(\alpha) \stackrel{c}{\rightarrow} \emptyset[\operatorname{guard}(\alpha) \wedge \psi] \text { where } c=\frac{d^{b}-1}{d-1}
$$

and let $\mathcal{P}^{\prime}=\mathcal{P} \cup\left\{\alpha^{\prime}\right\}$. Then $\mathcal{P}^{\prime}$ is well formed and the processor that maps $\mathcal{P}$ to $\mathcal{P}^{\prime}$ is sound.

Proof. Well-formedness is trivial due to the empty right-hand side $\emptyset$ of the rule $\alpha^{\prime}$. To prove soundness, note that by Theorem 4.3, $\sigma=\alpha^{\prime}$ implies $\operatorname{lhs}(\alpha) \sigma \stackrel{k}{\rightarrow} \alpha_{\alpha}^{m} S$ with $m \geq \frac{d^{b \sigma}-1}{d-1}$ for some cost $k$ and some configuration $S$ (since $\sigma \models \operatorname{guard}\left(\alpha^{\prime}\right)$ implies $\sigma \mid=\psi$, which in turn implies $(\llbracket \psi \rrbracket$. b) $\sigma=b \sigma)$. Since guard $(\alpha) \Longrightarrow \operatorname{cost}(\alpha) \geq 1$ is valid, we get $k \geq m \geq \frac{d^{b \sigma}-1}{d-1}=c \sigma$. Thus, for every evaluation with $\alpha^{\prime}$, there is an evaluation with $\alpha$ that has at least the same cost.

Example 4.6 (Accelerating Fibonacci). Since the rule (2) from Example 2.1 has cost 1, the implication (24) is trivially valid. Thus, accelerating the rule (2) yields

$$
\mathrm{fib}(x) \stackrel{2^{\frac{1}{2} x-1}-1}{\longrightarrow} \emptyset[x>1] .
$$

Afterwards, chaining this rule with rule (1) and deleting all other rules yields the simplified program with the rule

$$
\mathrm{f}_{0}(x) \stackrel{2^{\frac{1}{2} x-1}-1}{\longrightarrow} \emptyset[x>1] .
$$

\subsection{Simplifying Recursive Rules}

Section 4.2 showed how to accelerate simple recursions. If our implementation fails in applying Theorem 4.5 to a simple recursion, then we again try to eliminate temporary variables via Instantiation, as in the case of simple loops. If all temporary variables were eliminated and we still fail to accelerate a simple recursion, then further simplifications are possible. In particular, we can remove parts of the right-hand side via Partial Deletion.

Theorem 4.7 (Partial Deletion). Let $\mathcal{P}$ be a well-formed integer program and let $\alpha \in \mathcal{P}$. Moreover, let $\alpha^{\prime}$ be like $\alpha$, but $\operatorname{rhs}\left(\alpha^{\prime}\right) \subset \operatorname{rhs}(\alpha)$, and let $\mathcal{P}^{\prime}=\mathcal{P} \cup\left\{\alpha^{\prime}\right\}$. Then $\mathcal{P}^{\prime}$ is well formed and the processor that maps $\mathcal{P}$ to $\mathcal{P}^{\prime}$ is sound.

Proof. Since $\mathcal{P}$ is well formed, $\mathcal{P}^{\prime}$ is trivially well formed, too. To prove soundness, we define

$$
S \stackrel{k}{\rightarrow}{ }_{\mathcal{P}_{\circ} \supseteq} T \text { if } S \stackrel{k}{\rightarrow}{ }_{\mathcal{P}} \circ \supseteq T .
$$

So $S \stackrel{k}{\rightarrow}_{\mathcal{P}_{\circ \supset}} T$ holds if $S$ evaluates to some configuration $S^{\prime}$ with cost $k$ (i.e., $S \stackrel{k}{\rightarrow}_{\mathcal{P}} S^{\prime}$ ) and the configuration $T$ results from $S^{\prime}$ by deleting some terms from $T$ (i.e., $S^{\prime} \supseteq T$ ). Then, we clearly have $\operatorname{dh}_{\mathcal{P}^{\prime}}(S) \leq \sup \left\{k \in \mathbb{R} \mid S \stackrel{k^{\prime}}{\rightarrow} \mathcal{P}_{\circ \supseteq} T\right.$ for some $\left.T \in C\right\}$, as each $\rightarrow_{\mathcal{P}^{\prime}}$-sequence is also a $\rightarrow \mathcal{P}_{\circ \beth^{-}}$ sequence. To finish the proof, we show

$$
\sup \left\{k \in \mathbb{R} \mid S \stackrel{k}{\rightarrow} \stackrel{\mathcal{P}}{\circ} \supseteq^{*} T \text { for some } T \in C\right\} \leq \operatorname{dh} \mathcal{P}(S) .
$$

This clearly implies $\operatorname{rc} \mathcal{P}^{\prime}(n) \leq \operatorname{rc} \mathcal{P}(n)$, i.e., it implies that the processor is sound.

\footnotetext{
${ }^{13}$ If the implication (24) is not valid, then one can simply add the constraint $\operatorname{cost}(\alpha) \geq 1$ to the guard of the rule, since adding constraints to the guard is always sound, as it can only decrease the derivation height (by disallowing some evaluations).
} 
Consider an evaluation $S_{0} \stackrel{k_{1}}{\rightarrow}{ }_{\mathcal{P}}^{*} \supseteq \ldots \stackrel{k_{m}}{\longrightarrow} \underset{\mathcal{P}}{*} \supseteq S_{m}$. We prove $S_{0} \stackrel{k_{1}}{\longrightarrow} \underset{\mathcal{P}}{*} \ldots \stackrel{k_{m}}{\rightarrow}{ }_{\mathcal{P}}^{*} S_{m}^{\prime} \supseteq S_{m}$ for some $S_{m}^{\prime} \in C$ by induction on $m$. The case $m=0$ is trivial. If $m>0$, then the induction hypothesis implies

$$
S_{0} \stackrel{k_{1}}{\longrightarrow} \stackrel{*}{P} \ldots \stackrel{k_{m-1}}{\longrightarrow} \stackrel{*}{\mathcal{P}} S_{m-1}^{\prime} \supseteq S_{m-1} \text { for some } S_{m-1}^{\prime} \in C .
$$

Moreover, $S_{m-1} \stackrel{k_{m}}{\longrightarrow} \mathcal{P}_{\supset \supseteq} S_{m}$ implies $S_{m-1} \stackrel{k_{m}}{\longrightarrow} \widetilde{\mathcal{S}} \widetilde{S_{m}} \supseteq S_{m}$ for some $\widetilde{S_{m}} \in C$, i.e., we have $s \stackrel{k_{m}}{\longrightarrow} \mathcal{P} Q$ and $\widetilde{S_{m}}=\left(S_{m-1} \backslash\{s\}\right) \cup Q$ for some $s \in S_{m-1}$ and some $Q \in C$. Since $S_{m-1} \subseteq S_{m-1}^{\prime}$, we get

$$
S_{m-1}^{\prime}{\stackrel{k_{m}}{\longrightarrow}}_{\mathcal{P}}\left(S_{m-1}^{\prime} \backslash\{s\}\right) \cup Q=S_{m}^{\prime}
$$

and thus $S_{0} \stackrel{k_{1} *}{\longrightarrow} \underset{\mathcal{P}}{*} \ldots \stackrel{k_{m}}{\rightarrow}{ }_{\mathcal{P}}^{*} S_{m}^{\prime}$ with $S_{m}^{\prime} \supseteq \widetilde{S_{m}} \supseteq S_{m}$, as desired.

Example 4.8 (Partial Deletion to Enable Recursion Acceleration). Consider the simple recursion $\mathrm{f}(x, y) \rightarrow\{\mathrm{f}(x-1, y), \mathrm{f}(x-y, y)\}[x>0 \wedge y>x]$. As $\mathrm{f}(x-y, y)$ cannot be reduced any further if $y>x$, we cannot find a useful metering function for this rule and hence, Recursion Acceleration fails. By applying Partial Deletion, we obtain the simple loop $\mathrm{f}(x, y) \rightarrow \mathrm{f}(x-1, y)[x>0 \wedge y>x]$, which can easily be accelerated via Theorem 3.8. In this way, we can infer that the original nontail-recursive rule can be applied at least linearly often.

As shown above, Recursion Acceleration is useful to handle programs with non-linear recursion like the Fibonacci program, where the result is composed of two recursive calls. However, nontail-recursion also occurs when composing a recursive call with the call of an auxiliary function.

Example 4.9 (Non-tail-recursive facSum Program [14]). Consider the following imperative program:

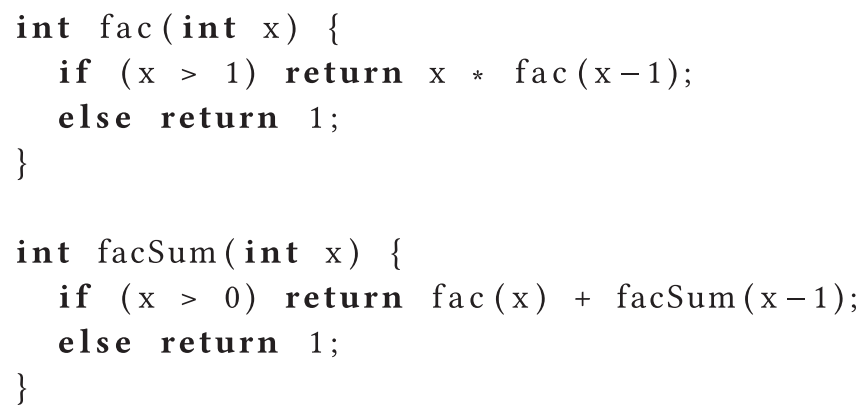

Here, fac $(\mathrm{x})$ computes $\mathrm{x}$ ! and facSum $(\mathrm{x})$ computes $0 !+\cdots+\mathrm{x}$ !. The program is not tail-recursive, because the last action of facSum is not the recursive call, but an addition. The integer program below represents its recursive structure, i.e., it can be obtained from the above program by a suitable abstraction.

$$
\begin{array}{rlr}
\mathrm{f}_{0}(x) & \stackrel{0}{\rightarrow} \operatorname{facSum}(x) & \\
\operatorname{facSum}(x) \stackrel{1}{\rightarrow}\{\mathrm{fac}(x), \operatorname{facSum}(x-1)\} & {[x>0]} \\
\operatorname{facSum}(x) \stackrel{1}{\rightarrow} \emptyset & {[x \leq 0]} \\
\operatorname{fac}(x) \stackrel{1}{\rightarrow} \operatorname{fac}(x-1) & {[x>1]} \\
\operatorname{fac}(x) \stackrel{1}{\rightarrow} \emptyset & {[x \leq 1] .}
\end{array}
$$

To analyze this integer program, we first accelerate and chain the recursive rule (28) as in Theorem 3.8 and Theorem 3.18. 
Example 4.10 (Accelerating fac). Clearly, $x-1$ is a metering function for the rule (28). Accelerating it using this metering function yields

$$
\mathrm{fac}(x) \stackrel{t v}{\longrightarrow} \mathrm{fac}(x-t v)[x>1 \wedge 0<t v<x] .
$$

Instantiating $t v$ with $x-1$ via Theorem 3.12 results in

$$
\mathrm{fac}(x) \stackrel{x-1}{\longrightarrow} \mathrm{fac}(1)[x>1] .
$$

At this point, we would like to chain the recursive facSum-rule (27) with the fac-rule (30). However, Theorem 3.18 is only applicable to tail-recursive rules. Hence, we now generalize Theorem 3.18 to arbitrary rules.

Theorem 4.11 (Chaining for Arbitrary Integer Programs). Let $\mathcal{P}$ be a well-formed integer program and let $\alpha_{1}, \alpha_{2} \in \mathcal{P}$ where

$$
\begin{array}{lllll}
\alpha_{1}: & f_{1}(\bar{x}) & \stackrel{c_{1}}{\longrightarrow} & S & {\left[\varphi_{1}\right] \text { with } f_{2}(\bar{x}) \mu \in S \text { and }} \\
\alpha_{2}: & f_{2}(\bar{x}) & \stackrel{c_{2}}{\longrightarrow} & T & {\left[\varphi_{2}\right] .}
\end{array}
$$

W.l.o.g., let $\mathcal{T} \mathcal{V}\left(\alpha_{1}\right) \cap \mathcal{T} \mathcal{V}\left(\alpha_{2}\right)=\emptyset$ (otherwise, the temporary variables in $\alpha_{2}$ can be renamed accordingly). Moreover, let $\alpha_{1.2}$ be the rule

$$
f_{1}(\bar{x}) \stackrel{c_{1}+c_{2} \mu}{\longrightarrow}\left(S \backslash\left\{f_{2}(\bar{x}) \mu\right\}\right) \cup T \mu\left[\varphi_{1} \wedge \varphi_{2} \mu\right],
$$

and let $\mathcal{P}^{\prime}=\mathcal{P} \cup\left\{\alpha_{1.2}\right\}$. Then $\mathcal{P}^{\prime}$ is well formed and the processor that maps $\mathcal{P}$ to $\mathcal{P}^{\prime}$ is sound.

Proof. To prove the soundness of the processor, we show that every evaluation step with $\alpha_{1.2}$ can be simulated by two evaluation steps with the rules $\alpha_{1}, \alpha_{2}$ of the same cost. Let $\sigma$ be an integer substitution with $\sigma \mid=\alpha_{1.2}$. Then, we have

$$
f_{1}(\bar{x}) \sigma \stackrel{c_{1} \sigma+c_{2} \mu \sigma}{\longrightarrow} \alpha_{1.2}\left(S \sigma \backslash\left\{f_{2}(\bar{x}) \mu \sigma\right\}\right) \cup T \mu \sigma .
$$

Since $\sigma \mid=\varphi_{1}$, we have

$$
f_{1}(\bar{x}) \sigma \stackrel{c_{1} \sigma}{\longrightarrow} \alpha_{1} S \sigma
$$

Since $\sigma \mid=\varphi_{2} \mu$ implies $\mu \circ \sigma \mid=\varphi_{2}$, we have

$$
f_{2}(\bar{x}) \mu \sigma \stackrel{c_{2} \mu \sigma}{\longrightarrow} \alpha_{2} T \mu \sigma .
$$

As $f_{2}(\bar{x}) \mu \in S$, this implies

$$
S \sigma \stackrel{c_{2} \mu \sigma}{\longrightarrow} \alpha_{2}\left(S \sigma \backslash\left\{f_{2}(\bar{x}) \mu \sigma\right\}\right) \cup T \mu \sigma .
$$

Thus, we have $f_{1}(\bar{x}) \sigma \stackrel{c_{1} \sigma+c_{2} \mu \sigma}{\longrightarrow} \underset{P}{2}\left(S \sigma \backslash\left\{f_{2}(\bar{x}) \mu \sigma\right\}\right) \cup T \mu \sigma$, as desired. As $\alpha_{1}$ and $\alpha_{2}$ are well formed, this also proves that $\mathcal{P}^{\prime}$ is well formed.

Note that Theorem 4.11 coincides with Theorem 3.18 if the degree of $\alpha_{1}$ and $\alpha_{2}$ is 1 . Theorem 4.11 allows us to continue the transformation of the program in Example 4.10.

Example 4.12 (Chaining facSum and fac). Chaining the recursive facSum-rule (27) of Example 4.10 with the accelerated fac-rule (30) yields

$$
\operatorname{facSum}(x) \stackrel{x}{\rightarrow}\{\operatorname{facSum}(x-1), \operatorname{fac}(1)\}[x>1] .
$$

Chaining this rule with the non-recursive fac-rule (29) results in

$$
\operatorname{facSum}(x) \stackrel{x+1}{\longrightarrow} \operatorname{facSum}(x-1)[x>1] .
$$

The iterated update and cost of this rule are $x \mu^{t v}=x-t v$ and

$$
\sum_{i=0}^{t v-1}(1+x) \mu^{i}=\sum_{i=0}^{t v-1}(1+x-i)=x \cdot t v-\frac{1}{2} t v^{2}+\frac{3}{2} t v .
$$


Thus, accelerating it via Theorem 3.8 with the metering function $x-1$ results in

$$
\operatorname{facSum}(x) \stackrel{x \cdot t v-\frac{1}{2} t v^{2}+\frac{3}{2} t v}{\longrightarrow} \operatorname{facSum}(x-t v)[0<t v<x],
$$

since $0<t v<x$ implies $x>1$. By instantiating $t v$ with $x-1$ and chaining rule (26) with the resulting rule, we obtain

$$
f_{0}(x) \stackrel{\frac{1}{2} x^{2}+\frac{3}{2} x-2}{\longrightarrow} \text { facSum }(1)[1<x] .
$$

Finally, by deleting all other rules, we obtain a simplified program.

Algorithm 2 shows how Algorithm 1 can be adapted to handle non-tail-recursive programs as well. The first additional step is Step 2, which deletes sinks (i.e., function symbols without any rules) from right-hand sides of non-tail-recursive rules $\alpha$. This simplifies the rules and possibly even transforms them into tail-recursive rules. ${ }^{14}$ Note that Partial Deletion adds a new rule $\alpha^{\prime}$ with fewer terms in its right-hand side (thus, we then delete the original rule $\alpha$ afterwards).

The second change is that we apply Partial Deletion in Step 3.3 if we failed to accelerate a simple recursion that does not contain any temporary variables anymore. In this way, the degree of the simple recursion is reduced, which may simplify its acceleration as in Example 4.8. In our implementation, we first try all partial deletions that result in rules of degree 2 (if any). If we fail to accelerate any of the resulting rules, then we also try all partial deletions that result in rules of degree 1.

Algorithm 2 terminates and thus it transforms any integer program into a simplified program: The loop in Step 2 reduces the degree of some rule in each iteration. The loop in Step 3 either reduces the number of non-accelerated loops or recursions or it reduces the number of temporary variables or the degree of some rule in each iteration. In Step 5, the number of accelerated rules is decreasing. The loop in Step 7 terminates as it reduces the number of function symbols with outgoing rules in each iteration. Finally, the overall loop of Algorithm 2 terminates as well, because after having finished Step 7 the first time, the number of function symbols decreases in each further iteration of the algorithm. To see this, note that there is no simple loop or simple recursion anymore when the loop of Step 5 terminates. Thus, after finishing the loop of Step 7, the only function symbol is either $\mathrm{f}_{0}$ (and hence the overall loop terminates) or there is at least one function symbol $f \neq \mathrm{f}_{0}$ without incoming or without outgoing rules. Thus, in the next iteration, this function symbol is removed. The reason is that if $f$ has no incoming rules, then all rules $\alpha$ with $\operatorname{root}(\alpha)=f$ are deleted in Step 1. If $f$ has no outgoing rules, then all occurrences of $f$ in right-hand sides are deleted in Step 2.

\section{ASYMPTOTIC LOWER BOUNDS}

After applying Algorithm 2, all programs are simplified and thus, we assume that $\mathcal{P}$ is a simplified program throughout this section. So all rules $\alpha \in \mathcal{P}$ have the same left-hand side $\mathrm{f}_{0}(\bar{x})$. Now for any integer substitution $\sigma$, the derivation height of $\mathrm{f}_{0}(\bar{x}) \sigma$ in the simplified program $\mathcal{P}$ is

$$
\operatorname{dh}_{\mathcal{P}}\left(\mathrm{f}_{0}(\bar{x}) \sigma\right)=\max \{\operatorname{cost}(\alpha) \sigma|\alpha \in \mathcal{P}, \sigma|=\alpha\},
$$

i.e., the derivation height (33) is the maximal cost of those rules whose guard is satisfied by $\sigma$. Thus, if $\mathcal{P}$ results from the transformation of an integer program $\widetilde{\mathcal{P}}$, then the derivation height (33) is a lower bound on $\operatorname{dh}_{\widetilde{\mathcal{P}}}\left(\mathrm{f}_{0}(\bar{x}) \sigma\right)$. So for the program in Figure $1(\mathrm{~b})$, which was transformed into the simplified program with the only rule

$$
\alpha_{0 . \overline{1} \cdot 2 \cdot \overline{3 . \overline{4} .5}}: \mathrm{f}_{0}(x, y, z, u) \stackrel{\frac{1}{8} x^{4}+\frac{1}{4} x^{3}+\frac{7}{8} x^{2}+\frac{7}{4} x}{\longrightarrow} \mathrm{f}_{2}\left(0, \frac{1}{2} x^{2}+\frac{1}{2} x, 1,0\right)\left[\frac{1}{2} x^{2}+\frac{1}{2} x>1\right],
$$

\footnotetext{
${ }^{14}$ If the rule is already tail-recursive, i.e., the right-hand side only contains a single term, and this term is a sink, then deleting this term does not help much to simplify the program. As mentioned in Footnote 3, we transform rules with empty right-hand side into rules with the right-hand side "sink" to simplify the formalization.
} 


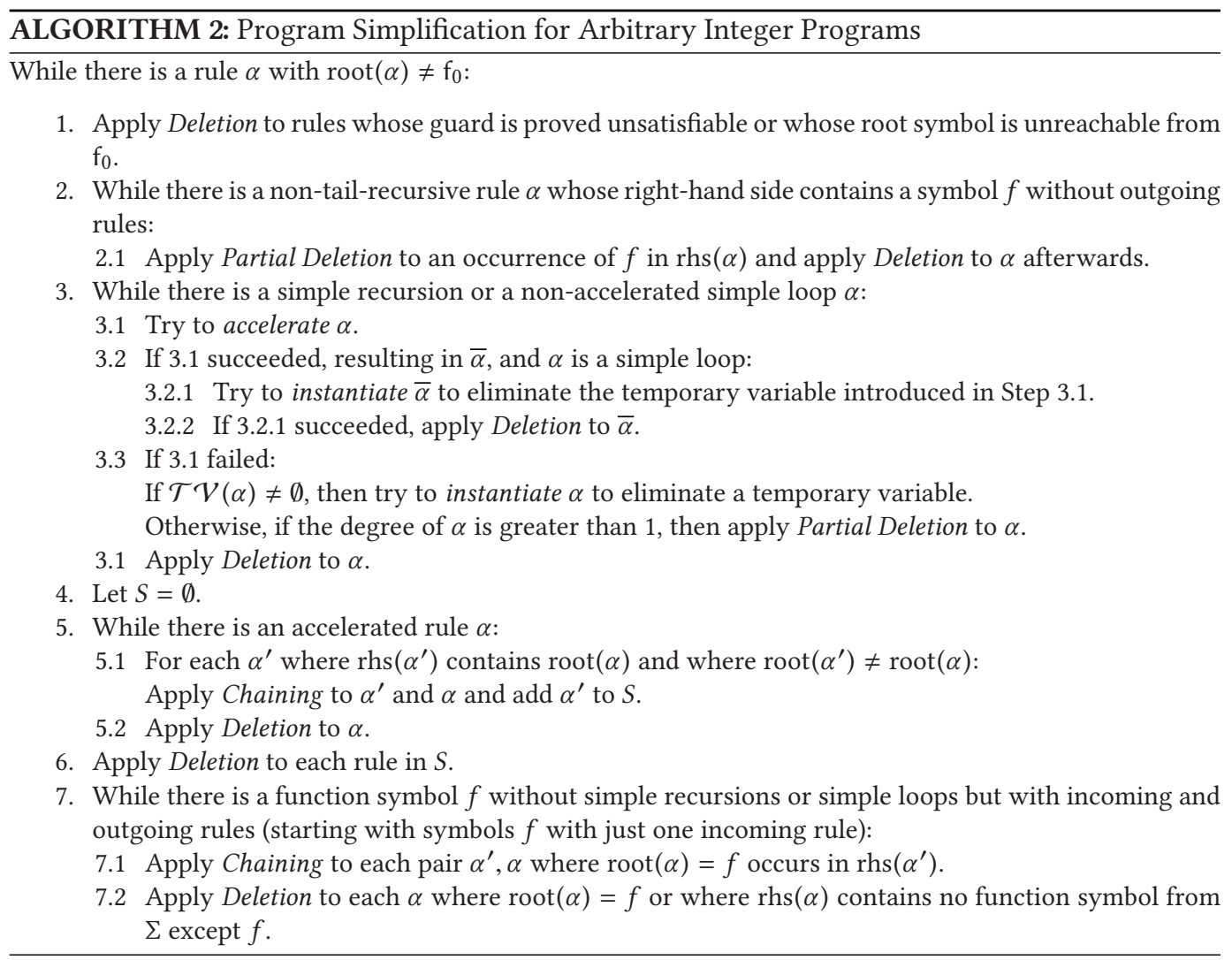

we obtain the lower bound

$$
\frac{1}{8} x^{4}+\frac{1}{4} x^{3}+\frac{7}{8} x^{2}+\frac{7}{4} x
$$

for all integer substitutions with $\sigma \mid=\frac{1}{2} x^{2}+\frac{1}{2} x>1$. However, in general such bounds do not provide an intuitive understanding of the program's complexity and they are also not suitable to detect possible attacks. The reason is that both $\operatorname{cost}(\alpha)$ and guard $(\alpha)$ may be complicated and, even more importantly, they may be interdependent. Then, it is not sufficient to only regard the cost of a rule to draw conclusions on the resulting complexity. To see this, consider a simplified rule with cost $t v$ and guard $\varphi$. Its complexity can, e.g., be unbounded if $\varphi$ does not impose any bound on $t v$, exponential if $\varphi$ only implies $t v \leq b$ for arithmetic expressions $b$ that are exponential in the program variables, or constant if $\varphi$ implies $t v \leq e$ for some $e \in \mathbb{N}$. But even without temporary variables, there can be subtle interdependencies between the cost and the guard of a transition, as the following example illustrates:

Example 5.1 (Sub-linear Bounds). Let

$$
\mathcal{P}=\left\{\mathrm{f}_{0}(x, y) \stackrel{y}{\rightarrow} \mathrm{f}(x, y)\left[x>y^{2}\right]\right\} .
$$

The runtime complexity of this program is sub-linear, even though the cost function $y$ is linear. The reason is that to achieve a linear increase of the cost $y$, a quadratic increase of $x$ and thus of the input size is required. So in general, it is not correct to simply take the cost of a rule as a lower bound on its runtime (e.g., in this example, we have $\operatorname{rc} \mathcal{P}(n) \in \Omega(\sqrt{n})$, whereas " $\operatorname{rc} \mathcal{P}(n) \in \Omega(n)$ " would be incorrect). 
Hence, we now show how to derive asymptotic lower bounds for simplified programs. These asymptotic bounds can easily be understood (i.e., a high lower bound can help programmers to improve their program to make it more efficient) and they identify potential attacks.

To derive asymptotic bounds, we use so-called limit problems, which are introduced in Section 5.1. A limit problem is an abstraction of the guard $\varphi$ of a rule that allows us to analyze how to satisfy $\varphi$, presuming that all variables are instantiated with "large enough" values. More precisely, a solution of a limit problem is a family of substitutions $\sigma_{n}$ that is parameterized by a variable $n$. This family of substitutions satisfies $\varphi$ for large enough $n$ and can be found using the calculus presented in Section 5.2. Thus, applying $\sigma_{n}$ to the cost of a rule yields an expression that only contains the single variable $n$, even if the rule has a multivariate cost function. Hence, this allows us to deduce an asymptotic bound. In Section 5.3, we present an alternative approach to find solutions of limit problems via SMT solving that can be combined with the calculus of Section 5.2 to improve efficiency.

\subsection{Limit Problems}

While the derivation height $\mathrm{dh}_{\mathcal{P}}$ is defined on configurations like $\mathrm{f}_{0}(\bar{x}) \sigma$, asymptotic bounds are usually defined for functions on $\mathbb{N}$ like the runtime complexity $\operatorname{rc} \mathcal{p}$. Recall that according to Definition $2.8, \operatorname{rc} \mathcal{P}(n)$ is the maximal cost of any evaluation starting with a configuration $\mathrm{f}_{0}(\bar{n})$ where the size $|\bar{n}|$ of the input is at most $n$. Thus, our goal is to derive an asymptotic lower bound for $\operatorname{rc} \mathcal{P}$ from a concrete bound on $\mathrm{dh}_{\mathcal{P}}$ like the lower bound (34). So for the program $\mathcal{P}$ with rule (19), we would like to derive $\operatorname{rc} \mathcal{P}(n) \in \Omega\left(n^{4}\right)$. However, as discussed above, in general the cost (34) of a rule does not directly give rise to the desired asymptotic lower bound.

To infer an asymptotic lower bound from a rule $\alpha \in \mathcal{P}$, we try to find an infinite family of integer substitutions $\sigma_{n}$ with $\mathcal{V}(\alpha) \subseteq \operatorname{dom}\left(\sigma_{n}\right)$ (parameterized by $n \in \mathbb{N}$ ) such that there is an $n_{0} \in \mathbb{N}$ with $\sigma_{n}=\operatorname{guard}(\alpha)$ for all $n \geq n_{0}$. Note that both $\left|\bar{x} \sigma_{n}\right|$ and $\operatorname{cost}(\alpha) \sigma_{n}$ are arithmetic expressions that only contain the single variable $n$, and we have $\operatorname{rc} \mathcal{P}\left(\left|\bar{x} \sigma_{n}\right|\right) \in \Omega\left(\operatorname{cost}(\alpha) \sigma_{n}\right)$, since for all $n \geq n_{0}$, we obtain

$$
\operatorname{rc} \mathcal{P}\left(\left|\bar{x} \sigma_{n}\right|\right) \geq \operatorname{dh} \mathcal{P}\left(\mathrm{f}_{0}(\bar{x}) \sigma_{n}\right) \geq \operatorname{cost}(\alpha) \sigma_{n} .
$$

For the program $\mathcal{P}$ containing only the rule

$$
\alpha_{0 . \overline{1} .2 . \overline{3 . \overline{4} .5}}: \mathrm{f}_{0}(x, y, z, u) \stackrel{\frac{1}{8} x^{4}+\frac{1}{4} x^{3}+\frac{7}{8} x^{2}+\frac{7}{4} x}{\longrightarrow} \mathrm{f}_{2}\left(0, \frac{1}{2} x^{2}+\frac{1}{2} x, 1,0\right)\left[\frac{1}{2} x^{2}+\frac{1}{2} x>1\right],
$$

our approach will infer the family $\sigma_{n}$ with

$$
x \sigma_{n}=n \quad \text { and } \quad y \sigma_{n}=z \sigma_{n}=u \sigma_{n}=0 .
$$

So for $\bar{x}$ consisting of $x, y, z, u$, this implies

$$
\begin{aligned}
\operatorname{rc} \mathcal{P}\left(\left|\bar{x} \sigma_{n}\right|\right) & =\operatorname{rc} \mathcal{P}\left(\left|x \sigma_{n}\right|+\left|y \sigma_{n}\right|+\left|z \sigma_{n}\right|+\left|u \sigma_{n}\right|\right) \\
& =\operatorname{rc} \mathcal{P}(|n|) \\
& \geq \operatorname{cost}\left(\alpha_{0 . \overline{1}} \cdot \overline{2.3 \cdot \overline{4} .5}\right) \sigma_{n} \\
& =(34) \sigma_{n} \\
& =\frac{1}{8} n^{4}+\frac{1}{4} n^{3}+\frac{7}{8} n^{2}+\frac{7}{4} n .
\end{aligned}
$$

To find such a family of substitutions $\sigma_{n}$, we first normalize all constraints in $\operatorname{guard}(\alpha)$ such that they have the form $a>0$ or $a \geq 0 .{ }^{15}$ Now, we search for substitutions $\sigma_{n}$ such that for large enough $n \in \mathbb{N}, \sigma_{n}$ is a model for a formula of the form " $\wedge_{i=1}^{k}\left(a_{i} \circ_{i} 0\right)$ " where $\circ_{i} \in\{>, \geq\}$. Obviously, such

\footnotetext{
${ }^{15}$ Note that while the variables range over $\mathbb{Z}$, there may be non-integer expressions in guard $(\alpha)$ that result from noninteger metering functions. Thus, we allow both constraints of the form $a>0$ and $a \geq 0$ in normalized guards, since transforming $a>0$ to $a-1 \geq 0$ would be incorrect in general.
} 
a formula is satisfied for large enough $n$ if all expressions $a_{i} \sigma_{n}$ are positive constants or increase infinitely towards $\omega$. Thus, we introduce a technique that tries to find out whether fixing the valuations of some variables and increasing or decreasing the valuations of others results in positive (respectively, increasing) valuations of $a_{1}, \ldots, a_{k}$. Our technique operates on limit problems of the form $\left\{a_{1}^{\bullet_{1}}, \ldots, a_{k}^{\bullet^{k}}\right\}$ where $a_{i}$ is an arithmetic expression and $\bullet_{i} \in\{+,-,+!,-!\}$ for all $1 \leq i \leq k$. Here, $a^{+}$(respectively, $a^{-}$) means that $a$ has to grow towards $\omega$ (respectively, $-\omega$ ) and $a^{+!}$(respectively, $a^{-!}$) means that $a$ has to be a positive (respectively, negative) constant. So, we represent $\operatorname{guard}(\alpha)$ by an initial limit problem $\left\{a_{1}^{\bullet_{1}}, \ldots, a_{k}^{\bullet_{k}}\right\}$ where $\bullet_{i} \in\{+,+!\}$ for all $1 \leq i \leq k$. By choosing $\bullet_{i}$ to be + or $+!, a_{i}^{\bullet^{i}}$ means that $a_{i}$ grows towards $\omega$ or it is a positive constant, i.e., this ensures that $a_{i}>0$ holds for large enough $n$. Our implementation leaves the choice of the $\bullet_{i} \in\{+,+$ ! $\}$ in the initial limit problem open as long as possible and only fixes it when this is needed to solve the limit problem (see the end of Section 5.2 for further details). To solve a limit problem $L$, we search for a solution $\sigma_{n}$ of $L$, where this concept is defined in terms of limits of functions.

Definition 5.2 (Limit). For each $f: \mathbb{N} \rightarrow \mathbb{R}$, we have $\lim _{n \mapsto \omega} f(n)=\omega$ (respectively, $\left.\lim _{n \mapsto \omega} f(n)=-\omega\right)$ if for every $m \in \mathbb{R}$ there is an $n_{0} \in \mathbb{N}$ such that $f(n) \geq m$ (respectively, $f(n) \leq m$ ) holds for all $n \geq n_{0}$. Similarly, we have $\lim _{n \mapsto \omega} f(n)=m$ if for every $\varepsilon \in \mathbb{R}$ with $\varepsilon>0$ there is an $n_{0} \in \mathbb{N}$ such that $|f(n)-m|<\varepsilon$ holds for all $n \geq n_{0}$.

Now a family of substitutions $\sigma_{n}$ is a solution for a limit problem $\left\{a_{1}^{\bullet_{1}}, \ldots, a_{k}^{\bullet^{k}}\right\}$ if $\lim _{n \mapsto \omega} a_{i} \sigma_{n}$ complies with $\bullet_{i}$ for each $1 \leq i \leq k$.

Definition 5.3 (Solutions of Limit Problems). For any function $f: \mathbb{N} \rightarrow \mathbb{R}$ and any $\bullet \in\{+,-,+!,-!\}$, we say that $f$ satisfies $\bullet$ if:

$$
\begin{array}{llll}
\lim _{n \mapsto \omega} f(n)=\omega, & \text { if } \bullet+ & \exists m \in \mathbb{R} . \lim _{n \mapsto \omega} f(n)=m>0, & \text { if } \bullet=+! \\
\lim _{n \mapsto \omega} f(n)=-\omega, & \text { if } \bullet=- & \exists m \in \mathbb{R} . \lim _{n \mapsto \omega} f(n)=m<0, & \text { if } \bullet=-!
\end{array}
$$

A family $\sigma_{n}$ of integer substitutions with $\mathcal{V}(L) \subseteq \operatorname{dom}\left(\sigma_{n}\right)$ is a solution of a limit problem $L$ if for every $a^{\bullet} \in L$, the function $\lambda n$. $a \sigma_{n}$ satisfies $\bullet$. For any arithmetic expression $a$ with $\mathcal{V}(a) \subseteq\{n\}$, " $\lambda n . a$ " is the function from $\mathbb{N} \rightarrow \mathbb{R}$ that maps any $n \in \mathbb{N}$ to the value of $a$.

Example 5.4 (Solution of the Limit Problem of the Program (19)). The program (19) has the guard $\frac{1}{2} x^{2}+\frac{1}{2} x>1$, which normalizes to $\frac{1}{2} x^{2}+\frac{1}{2} x-1>0$. Hence, the resulting initial limit problem could be $\left\{\left(\frac{1}{2} x^{2}+\frac{1}{2} x-1\right)^{+}\right\}$. It is solved by the family of substitutions $\sigma_{n}$ shown before (35). The reason is that $\lim _{n \mapsto \omega}\left(\lambda n\right.$. $\left.\left(\frac{1}{2} x^{2}+\frac{1}{2} x-1\right) \sigma_{n}\right)=\omega$, i.e., the function $\lambda n .\left(\frac{1}{2} x^{2}+\frac{1}{2} x-1\right) \sigma_{n}$ satisfies + . Thus, there is an $n_{0}$ such that $\sigma_{n}=\operatorname{guard}\left(\alpha_{0 . \overline{1} .2 . \overline{3 . \overline{4} .5}}\right)$ holds for all $n \geq n_{0}$.

In Sections 5.2 and 5.3, we will show how to infer such solutions of limit problems automatically. The following theorem clarifies how to deduce an asymptotic lower bound from a solution of a limit problem:

Theorem 5.5 (Asymptotic Bounds for Simplified Programs). Given a rule $\alpha$ of a simplified program $\mathcal{P}$ with the program variables $\bar{x}$ and $\operatorname{guard}(\alpha)=\left(a_{1} \circ_{1} 0\right) \wedge \cdots \wedge\left(a_{k} \circ \circ_{k} 0\right)$ where $\circ_{1}, \ldots, \circ_{k} \in\{>, \geq\}$, let the family $\sigma_{n}$ be a solution of an initial limit problem $\left\{a_{1}^{\bullet_{1}}, \ldots, a_{k}^{\bullet^{k}}\right\}$ with $\bullet_{1}, \ldots, \bullet_{k} \in\{+,+!\}$. Then $\operatorname{rc} \mathcal{P}\left(\left|\bar{x} \sigma_{n}\right|\right) \in \Omega\left(\operatorname{cost}(\alpha) \sigma_{n}\right)$.

Proof. Since $\sigma_{n}$ is a solution of $\left\{a_{1}^{\bullet_{1}}, \ldots, a_{k}^{\bullet}\right\}$, there is an $n_{0} \in \mathbb{N}$ such that for all $n \geq n_{0}$, we have $\sigma_{n}=a_{1}>0 \wedge \cdots \wedge a_{k}>0$, which implies $\sigma_{n}=\operatorname{guard}(\alpha)$. Hence, for all $n \geq n_{0}$, we obtain:

$$
\begin{aligned}
\operatorname{rc} \mathcal{P}\left(\left|\bar{x} \sigma_{n}\right|\right) & \geq \operatorname{dh} \mathcal{P}\left(\operatorname{lhs}(\alpha) \sigma_{n}\right) \\
& \geq \operatorname{cost}(\alpha) \sigma_{n} \quad \text { as } \sigma_{n} \mid=\operatorname{guard}(\alpha) .
\end{aligned}
$$

This implies $\operatorname{rc} \mathcal{P}\left(\left|\bar{x} \sigma_{n}\right|\right) \in \Omega\left(\operatorname{cost}(\alpha) \sigma_{n}\right)$. 
Of course, if $\mathcal{P}$ has several rules, then we try to take the one that results in the highest lower bound.

Example 5.6 (Asymptotic Bound for the Program (19)). We continue Example 5.4 with the program $\mathcal{P}=\{(19)\}$. For $\bar{x}=(x, y, z, u)$, according to Theorem 5.5, we get the asymptotic lower bound

$$
\operatorname{rc} \mathcal{P}\left(\left|\bar{x} \sigma_{n}\right|\right) \in \Omega\left(\operatorname{cost}((19)) \sigma_{n}\right) .
$$

Note that $\operatorname{cost}((19)) \sigma_{n}=\frac{1}{8} n^{4}+\frac{1}{4} n^{3}+\frac{7}{8} n^{2}+\frac{7}{4} n$. Hence, the asymptotic lower bound (36) is equivalent to

$$
\operatorname{rc} \mathcal{P}\left(\left|\bar{x} \sigma_{n}\right|\right) \in \Omega\left(n^{4}\right) .
$$

Up to now, we only took the guard $\bigwedge_{i=1}^{k}\left(a_{i} \circ_{i} 0\right)$ of a rule $\alpha$ into account in the initial limit problem $\left\{a_{1}^{\bullet_{1}}, \ldots, a_{k}^{\bullet^{k}}\right\}$. This has the disadvantage that solutions of this limit problem do not necessarily try to maximize the cost of the rule. For example, for the rule

$$
\mathrm{f}_{0}(x, y) \stackrel{x \cdot y}{\longrightarrow} \mathrm{f}(0, y)[x>0],
$$

we would obtain the initial limit problem $\left\{x^{+}\right\}$, which is solved by the family of substitutions $\sigma_{n}=\{x / n, y / 0\}$. According to Theorem 5.5, this only allows us to infer $\operatorname{rc} \mathcal{P}(n) \in \Omega\left(\operatorname{cost}(\alpha) \sigma_{n}\right)$, where $\operatorname{cost}(\alpha) \sigma_{n}=0$, i.e., it only allows us to infer a constant lower bound. To obtain non-trivial lower bounds instead, one should extend the initial limit problem $\left\{a_{1}^{\bullet_{1}}, \ldots, a_{k}^{\bullet^{k}}\right\}$ of a rule $\alpha$ by $\operatorname{cost}(\alpha)^{+}$. In this way, one searches for families of substitutions $\sigma_{n}$ where $\operatorname{cost}(\alpha) \sigma_{n}$ grows towards $\omega$, i.e., where $\operatorname{cost}(\alpha) \sigma_{n}$ depends on $n$ and is not constant. So in our example, we should start with the initial limit problem $\left\{x^{+},(x \cdot y)^{+}\right\}$, which has the solution $\sigma_{n}=\{x / n, y / n\}$. By Theorem 5.5, one now obtains the quadratic lower bound $\operatorname{rc} \mathcal{P}(n) \in \Omega\left(n^{2}\right)$, since $\operatorname{cost}(\alpha) \sigma_{n}=n^{2}$.

The costs are unbounded (i.e., they depend on temporary variables) if the initial limit problem $\left\{a_{1}^{\bullet_{1}}, \ldots, a_{k}^{\bullet^{k}}, \operatorname{cost}(\alpha)^{+}\right\}$has a solution $\sigma_{n}$ where $x \sigma_{n}$ is constant for all program variables $x$. Then, we can even infer $\operatorname{rc} \mathcal{P}(n) \in \Omega(\omega)$.

Example 5.7 (Unbounded Loops Continued). By chaining the initial rule $\mathrm{f}_{0}(x, y) \stackrel{0}{\rightarrow} \mathrm{f}(x, y)$ of the program from Example 3.6 with the accelerated rule

$$
\mathrm{f}(x, y) \stackrel{t v_{1} \cdot y}{\longrightarrow} \mathrm{f}\left(x+t v_{1}, y\right)\left[0<x \wedge 0<t v_{1}\right]
$$

from Example 3.11, we obtain

$$
\mathrm{f}_{0}(x, y) \stackrel{t v_{1} \cdot y}{\longrightarrow} \mathrm{f}\left(x+t v_{1}, y\right)\left[0<x \wedge 0<t v_{1}\right] .
$$

The resulting initial limit problem $\left\{x^{+!}, t v_{1}^{+},\left(t v_{1} \cdot y\right)^{+}\right\}$has the solution $\sigma_{n}=\left\{x / 1, y / 1, t v_{1} / n\right\}$, which implies $\operatorname{rc} \mathcal{P}(n) \in \Omega(\omega)$.

Theorem 5.5 results in bounds " $\operatorname{rc} \mathcal{P}\left(\left|\bar{x} \sigma_{n}\right|\right) \in \Omega\left(\operatorname{cost}(\alpha) \sigma_{n}\right)$," which depend on the sizes $\left|\bar{x} \sigma_{n}\right|$. Let $f(n)=\operatorname{rc} \mathcal{P}(n), g(n)=\left|\bar{x} \sigma_{n}\right|$, and let $\Omega\left(\operatorname{cost}(\alpha) \sigma_{n}\right)$ have the form $\Omega\left(n^{k}\right)$ or $\Omega\left(k^{n}\right)$ for some $k \in \mathbb{N}$. Moreover, for all $x \in \bar{x}$, let $x \sigma_{n}$ be a polynomial of at most degree $d$, i.e., let $g(n) \in O\left(n^{d}\right)$. Then, based on an observation from our paper [30], ${ }^{16}$ we can infer a lower bound for $f(n)=\operatorname{rc} \mathcal{P}(n)$ instead of $f(g(n))=\operatorname{rc} \mathcal{P}\left(\left|\bar{x} \sigma_{n}\right|\right)$. Moreover, if $g(n)=\left|\bar{x} \sigma_{n}\right|$ is constant whereas $\Omega\left(\operatorname{cost}(\alpha) \sigma_{n}\right)$ is not constant, then the lemma allows us to infer that $f(n)=\operatorname{rc} \mathcal{P}(n) \in \Omega(\omega)$, as in Example 5.7.

Lemma 5.8 (Bounds for Function Composition). Let $f: \mathbb{N} \rightarrow \mathbb{R}_{\geq 0} \cup\{\omega\}$ and $g: \mathbb{N} \rightarrow \mathbb{N}$ where $g(n) \in O\left(n^{d}\right)$ for some $d \in \mathbb{N}$ with $d>0$. Moreover, let $f(n)$ be weakly monotonically increasing for large enough $n$.

\footnotetext{
${ }^{16}$ In the second case of Lemma 5.8, we fix a small inaccuracy from our paper [29] where we inadvertently wrote $f(n) \in$ $\Omega\left(k^{d} \sqrt{n}\right)$. Since Lemma 5.8 is very similar to Lemma 24 from our paper [30], we omit its proof here. The proof can be found in Appendix A.
} 
(a) If $g(n)$ is strictly monotonically increasing for large enough $n$ and $f(g(n)) \in \Omega\left(n^{k}\right)$ with $k \in \mathbb{N}$, then $f(n) \in \Omega\left(n^{\frac{k}{d}}\right)$.

(b) If $g(n)$ is strictly monotonically increasing for large enough $n$ and $f(g(n)) \in \Omega\left(k^{n}\right)$ with $k>1$, then $f(n) \in \Omega\left(e^{\sqrt[d]{n}}\right)$ for some number $e \in \mathbb{R}$ with $e>1$.

(c) If $g(n) \in O(1)$ and $f(g(n)) \notin O(1)$, then $f(n) \in \Omega(\omega)$.

Example 5.9 (Asymptotic Bound for Program (19) Continued). In Example 5.6, for $\bar{x}=(x, y, z, u)$, we inferred $\operatorname{rc} \mathcal{P}\left(\left|\bar{x} \sigma_{n}\right|\right) \in \Omega\left(n^{4}\right)$ where $x \sigma_{n}=n$ and $y \sigma_{n}=z \sigma_{n}=u \sigma_{n}=0$. Hence, we have $\left|\bar{x} \sigma_{n}\right|=$ $|n|=n \in O\left(n^{1}\right)$. By Lemma 5.8(a), we obtain $\operatorname{rc} \mathcal{P}(n) \in \Omega\left(n^{\frac{4}{1}}\right)=\Omega\left(n^{4}\right)$ for the program $\mathcal{P}=\{(19)\}$. Due to the soundness of the processors for program simplification in Section 3, we also have $\operatorname{rc}_{\widetilde{\mathcal{P}}}(n) \in \Omega\left(n^{4}\right)$ for the program $\widetilde{\mathcal{P}}$ in Figure 1(b).

In cases like Example 5.1, Lemma 5.8 even allows us to infer sub-linear bounds.

Example 5.10 (Example 5.1 Continued). Reconsider the program from Example 5.1. By Definition 5.3, the family $\sigma_{n}$ with $x \sigma_{n}=n^{2}+1$ and $y \sigma_{n}=n$ is a solution of the initial limit problem $\left\{\left(x-y^{2}\right)^{+}, y^{+}\right\}$. (Our implementation chooses $\left(x-y^{2}\right)^{+!}$instead of $\left(x-y^{2}\right)^{+}$in the initial limit problem, because in this way, the limit problem can be solved by our technique; see Section 5.2.) Due to Theorem 5.5, this proves $\operatorname{rc} \mathcal{P}\left(\left|\bar{x} \sigma_{n}\right|\right) \in \Omega(n)$ for the program variables $\bar{x}=(x, y)$. As $\left|\bar{x} \sigma_{n}\right|=n^{2}+1+n \in O\left(n^{2}\right)$, Lemma 5.8(a) results in $\operatorname{rc} \mathcal{P}(n) \in \Omega\left(n^{\frac{1}{2}}\right)=\Omega(\sqrt{n})$.

If the cost of the rule from Example 5.1 was $2^{y}$, then $\sigma_{n}$ would still be a solution of the initial limit problem $\left\{\left(x-y^{2}\right)^{+!},\left(2^{y}\right)^{+}\right\}$. So, we would obtain $\operatorname{rc} \mathcal{P}\left(\left|\bar{x} \sigma_{n}\right|\right) \in \Omega\left(2^{n}\right)$ due to Theorem 5.5 and thus $\operatorname{rc} \mathcal{P}(n) \in \Omega\left(e^{\sqrt{n}}\right)$ for an $e>1$ due to Lemma 5.8(b). Intuitively, the exponent $\sqrt{n}$ expresses that the cost grows exponentially w.r.t. $y$, where the guard $x>y^{2}$ implies $|y| \in O(\sqrt{|\bar{x}|})$, i.e., $y$ is bounded by the square root of the input size.

The reason why we cannot specify $e$ in Lemma $5.8(\mathrm{~b})$ is that it depends on the coefficients of $g(n)=\left|\bar{x} \sigma_{n}\right|$, but Lemma 5.8 only requires $g(n) \in O\left(n^{d}\right)$. Thus, a variant of Lemma 5.8 where the polynomial $g$ is known would allow us to compute $e$. Our implementation simply reports that the runtime is at least exponential if Lemma 5.8(b) applies and $d=1$.

\subsection{Transforming Limit Problems}

To use Theorem 5.5 (and Lemma 5.8) for the automatic inference of lower bounds, we still have to show how to find a family of substitutions $\sigma_{n}$ automatically that solves the initial limit problem of a program's rule.

A limit problem $L$ is trivial if all expressions in $L$ are variables and there is no variable $x$ with $x^{\bullet}, x^{\bullet} \in L$ and $\bullet_{1} \neq \bullet_{2}$. For trivial limit problems $L$, we can immediately obtain a particular solution $\sigma_{n}^{L}$ that instantiates variables "according to $L$ ".

Lemma 5.11 (Solving Trivial Limit Problems). Let $L$ be a trivial limit problem. Then $\sigma_{n}^{L}$ is a solution of $L$ where for all $n \in \mathbb{N}$, $\sigma_{n}^{L}$ is defined as follows:

$$
x \sigma_{n}^{L}=\left\{\begin{aligned}
n & \text { if } x^{+} \in L \\
-n & \text { if } x^{-} \in L \\
1 & \text { if } x^{+!} \in L \\
-1 & \text { if } x^{-!} \in L \\
0 & \text { otherwise. }
\end{aligned}\right.
$$

Proof. If $x^{+} \in L$ (respectively, $x^{-} \in L$ ), then $x \sigma_{n}^{L}=n$ (respectively, $x \sigma_{n}^{L}=-n$ ) and thus, $\lim _{n \mapsto \omega} x \sigma_{n}=\lim _{n \mapsto \omega} n=\omega$ (respectively, $\lim _{n \mapsto \omega} x \sigma_{n}=\lim _{n \mapsto \omega}-n=-\omega$ ), i.e., $\lambda n$. $x \sigma_{n}$ satisfies $+\left(\right.$ respectively, - ). If $x^{+!} \in L$ (respectively, $x^{-!} \in L$ ), then $x \sigma_{n}^{L}=1$ (respectively, $x \sigma_{n}^{L}=-1$ ). Thus, 
$\lim _{n \mapsto \omega} x \sigma_{n}=1$ (respectively, $\lim _{n \mapsto \omega} x \sigma_{n}=-1$ ), i.e., $\lambda$ n. $x \sigma_{n}$ satisfies + ! (respectively, - !). Hence, $\sigma_{n}^{L}$ is a solution of $L$.

For instance, if $\mathcal{V}(\alpha)=\{x, y, t v\}$ and $L=\left\{x^{+}, y^{-}\right\}$, then $L$ is a trivial limit problem and $\sigma_{n}^{L}$ with $x \sigma_{n}^{L}=n, y \sigma_{n}^{L}=-1$, and $t v \sigma_{n}^{L}=0$ is a solution for $L$.

However, in general the initial limit problem $L=\left\{a_{1}^{\bullet}, \ldots, a_{k}^{{ }^{k}}, \operatorname{cost}(\alpha)^{+}\right\}$is not trivial. Therefore, we now define a transformation $\rightsquigarrow$ to simplify limit problems until one reaches a trivial problem. With our transformation, $L \rightsquigarrow L^{\prime}$ ensures that each solution of $L^{\prime}$ also gives rise to a solution of $L$.

If $L$ contains $f\left(a_{1}, a_{2}\right)^{\bullet}$ for some standard arithmetic operation $f$ like addition, subtraction, multiplication, division, or exponentiation, we use a so-called limit vector $\left(\bullet_{1}, \bullet_{2}\right)$ with $\bullet_{i} \in\{+,-,+$ !,- ! $\}$ to characterize for which kinds of arguments the operation $f$ is increasing (if $\bullet=+$ ), decreasing (if $\bullet=-$ ), or a positive or negative constant (if $\bullet=+$ ! or $\bullet=-$ !) ${ }^{17}$ Then $L$ can be transformed into the new limit problem $\left(L \backslash\left\{f\left(a_{1}, a_{2}\right)^{\bullet}\right\}\right) \cup\left\{a_{1}^{\bullet}, a_{2}^{\bullet}\right\}$.

For example, $(+,+!)$ and $(+,-!)$ are increasing limit vectors for subtraction. The reason is that $a_{1}-a_{2}$ is increasing if $a_{1}$ is increasing and $a_{2}$ is a constant. Hence, our transformation $\rightsquigarrow$ allows us to replace $\left(a_{1}-a_{2}\right)^{+}$by $a_{1}^{+}$and $a_{2}^{+!}$or by $a_{1}^{+}$and $a_{2}^{-!}$.

Definition 5.12 (Limit Vectors). Let $f: \mathbb{R} \rightarrow \mathbb{R}$ be a function and let $\bullet_{1}, \bullet_{2} \in\{+,-,+!,-$ ! $\}$. We say that $\left(\bullet_{1}, \bullet_{2}\right)$ is an increasing (respectively, decreasing) limit vector for $f$ if the function $\lambda n$. $f(g(n), h(n))$ satisfies $+\left(\right.$ respectively, - ) for any functions $g$ and $h$ that satisfy $\bullet_{1}$ and $\bullet_{2}$, respectively. Similarly, $\left(\bullet_{1}, \bullet_{2}\right)$ is a positive (respectively, negative) limit vector for $f$ if $\lambda n . f(g(n), h(n))$ satisfies + ! (respectively, - !) for any functions $g$ and $h$ that satisfy $\bullet_{1}$ and $\bullet_{2}$, respectively.

With this definition, $(+,+!)$ and $(+,-!)$ are indeed increasing limit vectors for subtraction, since $\lim _{n \mapsto \omega} g(n)=\omega$ and $\lim _{n \mapsto \omega} h(n)=m$ with $m>0$ or $m<0$ implies $\lim _{n \mapsto \omega}(g(n)-h(n))=\omega$. In other words, if $g(n)$ satisfies + and $h(n)$ satisfies + ! or - ! , then $g(n)-h(n)$ satisfies + as well. In contrast, $(+,+)$ is not an increasing limit vector for subtraction. To see this, consider the functions $g(n)=h(n)=n$. Both $g(n)$ and $h(n)$ satisfy + , whereas $g(n)-h(n)=0$ does not satisfy + . Similarly, $(+!,+!)$ is not a positive limit vector for subtraction, since for $g(n)=1$ and $h(n)=2$, both $g(n)$ and $h(n)$ satisfy + !, but $g(n)-h(n)=-1$ does not satisfy + !

Limit vectors can be used to simplify limit problems, as in (A) in the following definition. Moreover, for numbers $m \in \mathbb{R}$, one can easily simplify constraints of the form $m^{+!}$and $m^{-!}$(e.g., $2^{+!}$is obviously satisfied, since $2>0$ ), as in (B).

Definition $5.13(\rightsquigarrow)$. Let $L$ be a limit problem. We have:

(A) $L \cup\left\{f\left(a_{1}, a_{2}\right)^{\bullet}\right\} \rightsquigarrow L \cup\left\{a_{1}^{\bullet_{1}}, a_{2}^{\bullet_{2}}\right\}$ if $\bullet$ is $+($ respectively,,-+ !,- ! $)$ and $\left(\bullet_{1}, \bullet_{2}\right)$ is an increasing (respectively, decreasing, positive, negative) limit vector for $f$.

(B) $L \cup\left\{m^{+!}\right\} \rightsquigarrow L$ if $m \in \mathbb{R}$ and $m>0, L \cup\left\{m^{-!}\right\} \rightsquigarrow L$ if $m \in \mathbb{R}$ and $m<0$.

However, transforming a limit problem with $\rightsquigarrow$ may also result in contradictory limit problems that contain $x^{\bullet_{1}}$ and $x^{\bullet_{2}}$ where $\bullet_{1} \neq \bullet_{2}$, as the following example illustrates:

Example 5.14 (Contradictory Limit Problems-Example 5.10 Continued). The initial limit problem $\left\{\left(x-y^{2}\right)^{+!}, y^{+}\right\}$from Example 5.10 cannot be solved with the current transformation rules. While $(+,+!)$ and $(+,-!)$ are increasing limit vectors for subtraction, the only positive limit vector for subtraction is $(+!,-!)$. Thus, by $(\mathrm{A})$ one obtains $\left\{\left(x-y^{2}\right)^{+!}, y^{+}\right\} \rightsquigarrow\left\{x^{+!},\left(y^{2}\right)^{-!}, y^{+}\right\}$, which contains the unsolvable requirement $\left(y^{2}\right)^{-!}$.

\footnotetext{
${ }^{17}$ To ease the presentation, we restrict ourselves to binary operations $f$. For operations of arity $k$, one would need limit vectors of the form $\left(\bullet_{1}, \ldots, \bullet k\right)$.
} 
As an alternative, in Example 5.10 one could regard the initial limit problem $\left\{\left(x-y^{2}\right)^{+}, y^{+}\right\}$ instead. However, here the transformation rules fail as well. We have

$$
\begin{aligned}
& \left\{\left(x-y^{2}\right)^{+}, y^{+}\right\}, \\
\rightsquigarrow & \left\{x^{+},\left(y^{2}\right)^{+!}, y^{+}\right\} \text {by (A) with the increasing limit vector }(+,+!) \text { for subtraction, } \\
\rightsquigarrow & \left\{x^{+!}, y^{+!}, y^{+}\right\} \quad \text { by (A) with the positive limit vector }(+!,+!) \text { for multiplication. }
\end{aligned}
$$

However, the resulting problem is contradictory, as it contains both $y^{+!}$and $y^{+}$.

Recall that the guard of the rule from Example 5.1 implies $x \geq y^{2}+1$. If we substitute $x$ with its lower bound $y^{2}+1$ in the beginning, then we can reduce the initial limit problem $\left\{\left(x-y^{2}\right)^{+!}, y^{+}\right\}$ to a trivial one. Hence, we now extend $\rightsquigarrow$ by allowing to apply substitutions.

Definition 5.15 ( $\rightsquigarrow$ Continued). Let $L$ be a limit problem and let $\theta$ be a substitution such that $x \notin \mathcal{V}(x \theta)$ for all $x \in \operatorname{dom}(\theta)$ and $\theta \circ \sigma$ is an integer substitution for each integer substitution $\sigma$ whose domain includes all variables occurring in the range of $\theta$. Then, we have: ${ }^{18}$

(C) $L \stackrel{\theta}{\sim} L \theta$

Example 5.16 (Applying Substitutions to Limit Problems-Example 5.14 Continued). For the initial limit problem $\left\{\left(x-y^{2}\right)^{+!}, y^{+}\right\}$from Example 5.10, we now have

$$
\begin{array}{rll}
\left\{\left(x-y^{2}\right)^{+!}, y^{+}\right\} & \stackrel{\left\{x / y^{2}+1\right\}}{\longrightarrow} & \left\{1^{+!}, y^{+}\right\} \\
& \leadsto & \left\{y^{+}\right\}
\end{array}
$$

i.e., we obtain the trivial limit problem $\left\{y^{+}\right\}$. Note that, given an integer substitution $\sigma$ with $y \in$ $\operatorname{dom}(\sigma),\left\{x / y^{2}+1\right\} \circ \sigma$ is an integer substitution as well. By Lemma 5.11, the family $\sigma_{n}^{\left\{y^{+}\right\}}$with $y \sigma_{n}^{\left\{y^{+}\right\}}=n$ solves the resulting trivial limit problem $\left\{y^{+}\right\}$. To obtain a solution for the initial limit problem $\left\{\left(x-y^{2}\right)^{+!}, y^{+}\right\}$one has to take the substitution $\left\{x / y^{2}+1\right\}$ into account that was used in its transformation. In this way, we get the solution $\sigma_{n}=\left\{x / y^{2}+1\right\} \circ \sigma_{n}^{\left\{y^{+}\right\}}$for the initial limit problem where $x \sigma_{n}=n^{2}+1$ and $y \sigma_{n}=n$. Thus, we obtain $\operatorname{rc} \mathcal{P}(n) \in \Omega\left(n^{\frac{1}{2}}\right)=\Omega(\sqrt{n})$, as in Example 5.10.

Although Definition 5.15 requires that $\theta \circ \sigma$ is an integer substitution whenever $\sigma$ is an integer substitution, it is also useful to handle limit problems that contain expressions that do not evaluate to integer numbers.

Example 5.17 (Non-integer Metering Functions Continued). By chaining ${ }^{19}$ the only initial rule of the program in Example 3.10 with the accelerated rule (17), we obtain

$$
\mathrm{f}_{0}(x) \stackrel{t v}{\rightarrow} \mathrm{f}(x-2 t v)\left[0<t v<\frac{1}{2} x+1\right] .
$$

For the initial limit problem $\left\{t v^{+},\left(\frac{1}{2} x+1-t v\right)^{+!}\right\}$, we get

$$
\left\{t v^{+},\left(\frac{1}{2} x+1-t v\right)^{+!}\right\} \stackrel{\{x / 2 t v-1\}}{\leadsto} \begin{array}{ll}
\left\{t v^{+}, \frac{1}{2}^{+!}\right\} \\
& \left.\leadsto t v^{+}\right\}
\end{array}
$$

by (C) and (B). (Our implementation first leaves it open whether to choose $\left(\frac{1}{2} x+1-t v\right)^{+}$or $\left(\frac{1}{2} x+1-t v\right)^{+!}$, but when transforming the arithmetic expression to $\frac{1}{2}$ by $(\mathrm{C})$, it finds out that one should use $\left(\frac{1}{2} x+1-t v\right)^{+!}$to solve the limit problem. We describe the strategy used by our

\footnotetext{
${ }^{18}$ The other rules for $\rightsquigarrow$ are implicitly labeled with the identical substitution $\emptyset$.

${ }^{19}$ Note that we cannot instantiate $t v$ with the metering function that was used to accelerate the loop from Example 3.10, as it does not map to the integers, i.e., the prerequisites of Theorem 3.12 are not satisfied.
} 
implementation and its heuristic to find suitable substitutions $\theta$ for the application of rule (C) at the end of this subsection.) By Lemma 5.11, the family $\sigma_{n}^{\left\{t v^{+}\right\}}$with $t v \sigma_{n}^{\left\{t v^{+}\right\}}=n$ solves the resulting trivial limit problem $\left\{t v^{+}\right\}$. Again, to obtain a solution for the original initial limit problem $\left\{t v^{+},\left(\frac{1}{2} x+1-t v\right)^{+!}\right\}$one has to take the substitution $\{x / 2 t v-1\}$ into account, resulting in $\sigma_{n}$ with $x \sigma_{n}=2 n-1$ and $t v \sigma_{n}=n$. Thus, by Theorem 5.5, we have $\operatorname{rc}_{\{(37)\}}\left(\left|x \sigma_{n}\right|\right)=\operatorname{rc}_{\{(37)\}}(2 n-1) \in$ $\Omega\left(\operatorname{cost}((37)) \sigma_{n}\right)=\Omega\left(t v \sigma_{n}\right)=\Omega(n)$. As $2 n-1 \in O(n)$, Lemma 5.8(a) implies $\operatorname{rc}_{\{(37)\}}(n) \in \Omega(n)$. By the soundness of the processors for program simplification in Section 3, we also have $\operatorname{rc} \mathcal{P}(n) \in$ $\Omega(n)$ for the original program in Example 3.10.

Definition 5.15 requires that $\theta \circ \sigma$ is an integer substitution for every integer substitution $\sigma$ whose domain includes all variables occurring in the range of $\theta$. To check this side-condition automatically, one can again use Lemma 3.15: If the range of $\theta$ consists of polynomials, then for every $x \in \operatorname{dom}(\theta)$, we only have to check if instantiating the polynomial $x \theta$ by finitely many suitable integers again results in an integer. More precisely, if $x \theta$ contains the variables $x_{1}, \ldots, x_{k}$ of degrees $d_{1}, \ldots, d_{k}$, respectively, we check if $x \theta$ maps all arguments from $\left\{0, \ldots, d_{1}+1\right\} \times \cdots \times$ $\left\{0, \ldots, d_{k}+1\right\}$ to integers.

However, up to now, we cannot prove that, e.g., a rule $\alpha$ with $\operatorname{guard}(\alpha)=x^{2}-x>0$ and $\operatorname{cost}(\alpha)=x$ has a linear lower bound, since $(+,+)$ is not an increasing limit vector for subtraction. To handle such cases, the following transformation rules allow us to neglect polynomial sub-expressions if they are "dominated" by other polynomials of higher degree or by exponential sub-expressions.

Definition 5.18 ( $\rightsquigarrow$ Continued). Let $L$ be a limit problem, let $\pm \in\{+,-\}$, and let $a, b, c$ be univariate polynomials over the same variable. Then, we have:

(D) $L \cup\left\{(a \pm b)^{+}\right\} \rightsquigarrow L \cup\left\{a^{+}\right\}$and $L \cup\left\{(a \pm b)^{-}\right\} \rightsquigarrow L \cup\left\{a^{-}\right\}$if the degree of $a$ is greater than the degree of $b$.

(E) $L \cup\left\{\left(a^{c} \pm b\right)^{+}\right\} \rightsquigarrow L \cup\left\{(a-1)^{+}, c^{+}\right\}$and $L \cup\left\{\left(a^{c} \pm b\right)^{+}\right\} \rightsquigarrow L \cup\left\{(a-1)^{+}, c^{+!}\right\}$.

Thus, $\left\{\left(x^{2}-x\right)^{+}\right\} \rightsquigarrow\left\{\left(x^{2}\right)^{+}\right\}=\left\{(x \cdot x)^{+}\right\} \rightsquigarrow\left\{x^{+}\right\}$by (D) and (A) with the increasing limit vector $(+,+)$ for multiplication. The intuition for (E) is that any exponential expression $a^{c}$ dominates any polynomial expression $b$ provided that the base $a$ is greater than 1 and the exponent $c$ grows towards $\omega$.

Example 5.19 (Example 4.6 Continued). We continue Example 4.6, where the Fibonacci program was simplified to the program consisting just of the rule

$$
\mathrm{f}_{0}(x) \stackrel{2^{\frac{1}{2} x-1}-1}{\longrightarrow} \emptyset[x>1] .
$$

Here, we obtain the initial limit problem $\left\{(x-1)^{+},\left(2^{\frac{1}{2} x-1}-1\right)^{+}\right\}$. We get:

$$
\begin{aligned}
& \left\{(x-1)^{+},\left(2^{\frac{1}{2} x-1}-1\right)^{+}\right\} \\
& \rightsquigarrow\left\{x^{+},\left(2^{\frac{1}{2} x-1}-1\right)^{+}\right\} \quad \text { by }(\mathrm{D}) \\
& \rightsquigarrow\left\{x^{+}, 1^{+!},\left(\frac{1}{2} x-1\right)^{+}\right\} \quad \text { by }(\mathrm{E}) \\
& \rightsquigarrow\left\{x^{+}, 1^{+!},\left(\frac{1}{2} x\right)^{+}\right\} \quad \text { by }(\mathrm{A}) \text { with the increasing limit vector }(+,+!) \text { for subtraction } \\
& \rightsquigarrow\left\{x^{+}, 1^{+!},\left(\frac{1}{2}\right)^{+!}\right\} \quad \text { by }(\mathrm{A}) \text { with the increasing limit vector }(+!,+) \text { for multiplication } \\
& \rightsquigarrow^{2}\left\{x^{+}\right\} \quad \text { by (B) (twice). }
\end{aligned}
$$

By Lemma 5.11, the family $\sigma_{n}^{\left\{x^{+}\right\}}$with $x \sigma_{n}^{\left\{x^{+}\right\}}=n$ solves the resulting trivial limit problem $\left\{x^{+}\right\}$and hence, it also solves the initial limit problem of rule (25). Thus, Theorem 5.5 implies 
(A) $L \cup\left\{f\left(a_{1}, a_{2}\right)^{\bullet}\right\} \leadsto L \cup\left\{a_{1}^{\bullet}, a_{2}^{\bullet}\right\}$ if $\bullet$ is $+($ resp.,-+ !,$-!)$ and $\left(\bullet_{1}, \bullet_{2}\right)$ is an increasing (resp. decreasing, positive, negative) limit vector for $f$

(B) $L \cup\left\{m^{+!}\right\} \leadsto L$ if $m \in \mathbb{R}$ and $m>0, L \cup\left\{m^{-!}\right\} \leadsto L$ if $m \in \mathbb{R}$ and $m<0$

(C) $L \stackrel{\theta}{\rightarrow} L \theta$ if $x \notin \mathcal{V}(x \theta)$ for all $x \in \operatorname{dom}(\theta)$, and if $\sigma$ is an integer substitution with $\mathcal{V}($ range $(\theta)) \subseteq \operatorname{dom}(\sigma)$, then $\theta \circ \sigma$ is also an integer substitution

(D) $L \cup\left\{(a \pm b)^{\bullet}\right\} \leadsto L \cup\left\{a^{\bullet}\right\}$ if $\bullet \in\{+,-\}$ and degree $(a)>$ degree $(b)$ for the univariate polynomials $a, b$

(E) $L \cup\left\{\left(a^{c} \pm b\right)^{+}\right\} \leadsto L \cup\left\{(a-1)^{\bullet}, c^{+}\right\}$if $\bullet \in\{+,+!\}$ for the univariate polynomials $a, b, c$

Fig. 2. Definition of our transformation for a limit problem $L$.

$\operatorname{rc}_{\{(25)\}}\left(\left|x \sigma_{n}\right|\right)=\operatorname{rc}_{\{(25)\}}(n) \in \Omega\left(\operatorname{cost}((25)) \sigma_{n}\right)=\Omega\left(2^{\frac{1}{2} n-1}-1\right)=\Omega\left(2^{\frac{1}{2} n}\right)=\Omega\left(\sqrt{2}^{n}\right) \subset \Omega\left(1.4^{n}\right)$.

Due to the soundness of the program simplification in Section 3 and Section 4, this also implies that the runtime complexity of the original Fibonacci program $\mathcal{P}$ from Example 2.1 is exponential, i.e., $\operatorname{rc} \mathcal{P}(n) \in \Omega\left(\sqrt{2}^{n}\right)$.

Note that (E) can also be used to handle limit problems like $\left(a^{c}\right)^{+}$(by choosing $b=0$ ). We summarize the full definition of our transformation $\rightsquigarrow$ of limit problems in Figure 2. Theorem 5.20 states that our transformation $\rightsquigarrow$ is indeed correct. As already illustrated in Examples 5.16 and 5.17, when constructing the solution from the resulting trivial limit problem, one has to take the substitutions into account that were used in the derivation.

THEOREM 5.20 (CORRECTNESS OF $\rightsquigarrow$ ). If $L \stackrel{\theta}{\rightsquigarrow} L^{\prime}$ and the family $\sigma_{n}$ is a solution of $L^{\prime}$, then $\theta \circ \sigma_{n}$ is a solution of $L$.

Proof. First assume that the step from $L$ to $L^{\prime}$ was done by Definition 5.13 (A). Since $\sigma_{n}$ is a solution for $L^{\prime}$, it is a solution for $a_{1}^{\bullet_{1}}$ and $a_{2}^{\bullet}$, where $\left(\bullet_{1}, \bullet_{2}\right)$ is an increasing (respectively, decreasing, positive, or negative) limit vector for $f$. As $\sigma_{n}$ is a solution for both $a_{i}^{\bullet}$, the function $\lambda n$. $a_{i} \sigma_{n}$ satisfies $\bullet_{i}$. By the definition of limit vectors, this implies that $\lambda n . f\left(a_{1} \sigma_{n}, a_{2} \sigma_{n}\right)=\lambda n . f\left(a_{1}, a_{2}\right) \sigma_{n}$ satisfies $\bullet$. Thus, $\sigma_{n}$ is a solution for $f\left(a_{1}, a_{2}\right)^{\bullet}$.

If the step from $L$ to $L^{\prime}$ was done by Definition 5.13 (B), then every solution $\sigma_{n}$ for $L^{\prime}$ is also a solution for $L$, since $m \sigma_{n}=m$ holds for any $m \in \mathbb{R}$.

If the step from $L$ to $L^{\prime}$ was done by Definition $5.15(\mathrm{C})$, then let $\sigma_{n}$ be a solution for $L^{\prime}=L \theta$. Then for every $(a \theta)^{\bullet} \in L \theta, \lambda n$. $a \theta \sigma_{n}$ satisfies $\bullet$ and hence $\theta \circ \sigma_{n}$ is a solution for $a^{\bullet}$. Thus, $\theta \circ \sigma_{n}$ is a solution for $L$.

If the step from $L$ to $L^{\prime}$ was done by Definition 5.18 (D), then let $\sigma_{n}$ be a solution for $a^{\bullet}$. Since the polynomial $a$ only contains a single variable (say, $x$ ), we must have $\lim _{n \mapsto \omega} x \sigma_{n}=\omega$ or $\lim _{n \mapsto \omega} x \sigma_{n}=-\omega$. W.l.o.g, let $\lim _{n \mapsto \omega} x \sigma_{n}=\omega$ and $\bullet=+$ (the other cases work analogously). Then $\lim _{n \mapsto \omega} a \sigma_{n}=\omega$ implies $\lim _{x \mapsto \omega} a=\omega$. Since the degree of $a$ is greater than the degree of $b$, this means $\lim _{x \mapsto \omega}(a \pm b)=\omega$ and hence $\lim _{n \mapsto \omega}(a \pm b) \sigma_{n}=\omega$.

For Definition $5.18(\mathrm{E})$, the proof is analogous. Here for large enough $n, a^{c} \sigma_{n}$ is an exponential function with a base $>1$. Since $\sigma_{n}$ is a solution for $c^{+}$, we again have $\lim _{n \mapsto \omega} x \sigma_{n}=\omega$ or $\lim _{n \mapsto \omega} x \sigma_{n}=-\omega$. Thus, $a^{c} \sigma_{n}$ is an exponential function that grows faster than $b \sigma_{n}$ for $n \mapsto \omega$. Hence, we obtain $\lim _{n \mapsto \omega}\left(a^{c} \pm b\right) \sigma_{n}=\omega$.

So to find an asymptotic lower bound for the runtime of a simplified program with a rule $\alpha$, we start with an initial limit problem $L=\left\{a_{1}^{\bullet}, \ldots, a_{k}^{{ }^{*}}, \operatorname{cost}(\alpha)^{+}\right\}$that represents $\operatorname{guard}(\alpha)$ and requires non-constant cost, and transform $L$ with $\rightsquigarrow$ into a trivial limit problem $L^{\prime}$, i.e., $L \stackrel{\theta_{1}}{\rightsquigarrow} \ldots \stackrel{\theta_{m}}{\rightsquigarrow} L^{\prime}$. As mentioned before, for automation one should leave the $\bullet_{i}$ in the initial problem $L$ open, and 
only instantiate them by a value from $\{+,+!\}$ when this is needed to apply a particular rule of the transformation $\rightsquigarrow$. Then by Lemma 5.11 and Theorem 5.20 , the resulting family $\sigma_{n}^{L^{\prime}}$ of substitutions gives rise to a solution $\sigma_{n}=\theta_{1} \circ \cdots \circ \theta_{m} \circ \sigma_{n}^{L^{\prime}}$ of $L$. Thus, by Theorem 5.5, we have $\operatorname{rc} \mathcal{P}\left(\left|\bar{x} \sigma_{n}\right|\right) \in$ $\Omega\left(\operatorname{cost}(\alpha) \sigma_{n}\right)$, which leads to a lower bound for $\operatorname{rc} \mathcal{P}(n)$ with Lemma 5.8.

Our implementation uses the following strategy to apply the rules from Definitions 5.13, 5.15, and 5.18 for the transformation $\rightsquigarrow$. Initially, we reduce the number of variables by propagating bounds implied by the guard of the rule $\alpha$. For example, if an arithmetic expression $a$ with $x \notin \mathcal{V}(a)$ is a minimal upper or a maximal lower bound on $x$ (i.e., guard $(\alpha)$ implies $x \leq a$ but not $x \leq a-1$, or guard $(\alpha)$ implies $x \geq a$ but not $x \geq a+1)$, then we may apply the substitution $\{x / a\}$ to the initial limit problem by the rule (C). Thus, we can, e.g., simplify the limit problem from Example 5.10 by instantiating $x$ with $y^{2}+1$ (see Example 5.16). In the same way, the substitution that is applied in Example 5.17 can be found automatically. Afterwards, we use (B) and (D) with highest and (E) with second-highest priority. The third priority is trying to apply (A) to univariate expressions (since processing univariate expressions helps to guide the search). As fourth priority, we apply (C) with a suitable substitution $\{x / m\}$ if $x^{+!}$or $x^{-}$occurs in the current limit problem. Otherwise, we apply (A) to multivariate expressions. Since $\rightsquigarrow$ is well founded and, except for (C), finitely branching, one may also backtrack and explore alternative applications of $\rightsquigarrow$. In particular, we backtrack if we obtain a contradictory limit problem. Moreover, if we obtain a trivial limit problem $L^{\prime}$ where $\operatorname{cost}(\alpha) \sigma_{n}^{L^{\prime}}$ is a polynomial, but $\operatorname{cost}(\alpha)$ is a polynomial of higher degree or an exponential function, then we backtrack to search for other solutions that might lead to a higher lower bound. However, our implementation can of course fail, since solvability of limit problems is undecidable (due to Hilbert's Tenth Problem).

Example 5.21 (Solving the Limit Problem of the Program (19)). For the program $\mathcal{P}=\{(19)\}$ that results from the simplification of the program $\widetilde{\mathcal{P}}$ in Figure 1(b), we obtain the initial limit problem $\left\{\left(\frac{1}{2} x^{2}+\frac{1}{2} x-1\right)^{+},\left(\frac{1}{8} x^{4}+\frac{1}{4} x^{3}+\frac{7}{8} x^{2}+\frac{7}{4} x\right)^{+}\right\}$. Here, we have:

$$
\left\{\left(\frac{1}{2} x^{2}+\frac{1}{2} x-1\right)^{+},\left(\frac{1}{8} x^{4}+\frac{1}{4} x^{3}+\frac{7}{8} x^{2}+\frac{7}{4} x\right)^{+}\right\}
$$

$\stackrel{(D)}{\rightsquigarrow 2}\left\{\left(\frac{1}{2} x^{2}\right)^{+},\left(\frac{1}{8} x^{4}\right)^{+}\right\} \quad$ as the degree of $\frac{1}{2} x^{2}$ is greater than the degree of $\frac{1}{2} x-1$ and the degree of $\frac{1}{8} x^{4}$ is greater than the degree of $\frac{1}{4} x^{3}+\frac{7}{8} x^{2}+\frac{7}{4} x$

$\stackrel{(A)}{\leftrightarrow 2}\left\{\frac{1}{2}^{+!},\left(x^{2}\right)^{+}, \frac{1}{8}^{+!},\left(x^{4}\right)^{+}\right\}$using the increasing limit vector $(+!,+)$for multiplication

$\stackrel{(B)}{\rightarrow 2}\left\{\left(x^{2}\right)^{+},\left(x^{4}\right)^{+}\right\}$

$\stackrel{(A)}{\rightsquigarrow 2}\left\{x^{+}\right\} \quad$ using the increasing limit vector $(+,+)$ for multiplication.

The resulting trivial limit problem $\left\{x^{+}\right\}$gives rise to the solution (35) and hence proves $\operatorname{rc} \mathcal{P}\left(\left|\bar{x} \sigma_{n}\right|\right) \in$ $\Omega\left(n^{4}\right)$ for $\bar{x}=(x, y, z, u)$ (see Example 5.6). As $\left|\bar{x} \sigma_{n}\right| \in O(n)$, as in Example 5.9, we get $\operatorname{rc} \mathcal{P}(n) \in$ $\Omega\left(n^{4}\right)$ and thus also $\operatorname{rc}_{\widetilde{\rho}}(n) \in \Omega\left(n^{4}\right)$.

\subsection{Solving Limit Problems via SMT}

While the calculus presented in Section 5.2 permits a precise analysis of simplified programs, it can also be quite expensive in practice. The reason is that the next $\rightsquigarrow$-step is rarely unique and thus backtracking is often unavoidable to find a good lower bound. We now show how limit problems can be encoded as conjunctions of polynomial inequations. In many cases, this allows us to use SMT solvers to solve limit problems more efficiently. 
Essentially, the idea is to search for a solution $\sigma_{n}$ (i.e., a suitable family of substitutions) that instantiates each variable $x$ in the limit problem by a linear polynomial $x \sigma_{n}=m_{x} \cdot n+k_{x}$. Here, we leave the integers $m_{x}$ and $k_{x}$ open (i.e., they are abstract coefficients) and we use SMT solving to find an instantiation of the abstract coefficients by integer numbers such that $\sigma_{n}$ becomes a solution for the limit problem.

Thus, if $a$ is a polynomial arithmetic expression, then $a \sigma_{n}=a\left\{x /\left(m_{x} \cdot n+k_{x}\right) \mid x \in \mathcal{V}(a)\right\}$ is a univariate polynomial over $n$ with abstract coefficients. If $a$ is of degree $d$, then $a \sigma_{n}$ can be rearranged to the form $a_{d} \cdot n^{d}+\cdots+a_{0} \cdot n^{0}$ where the $a_{i}$ are arithmetic expressions over the abstract coefficients $\left\{m_{x}, k_{x} \mid x \in \mathcal{V}(a)\right\}$ that do not contain $n$.

Example 5.22 (Encoding the Initial Limit Problem for Program (19)). Consider the initial limit problem $\left\{\left(\frac{1}{2} x^{2}+\frac{1}{2} x-1\right)^{+}\right\}$for the program with the rule (19) (see Example 5.4). We use $\sigma_{n}$ with $x \sigma_{n}=m_{x} \cdot n+k_{x}$. Therefore, we obtain

$$
\begin{aligned}
\left(\frac{1}{2} x^{2}+\frac{1}{2} x-1\right) \sigma_{n} & =\frac{1}{2} \cdot\left(m_{x} \cdot n+k_{x}\right)^{2}+\frac{1}{2} \cdot\left(m_{x} \cdot n+k_{x}\right)-1 \\
& =a_{2} \cdot n^{2}+a_{1} \cdot n+a_{0},
\end{aligned}
$$

where $a_{2}=\frac{1}{2} m_{x}^{2}, a_{1}=m_{x} \cdot k_{x}+\frac{1}{2} m_{x}$, and $a_{0}=\frac{1}{2} k_{x}^{2}+\frac{1}{2} k_{x}-1$.

Clearly, we have $\lim _{n \mapsto \omega} a \sigma_{n}=\omega$ (respectively, $-\omega$ ) if and only if $a_{i}>0$ (respectively, $a_{i}<0$ ) for some $i>0$ and $a_{j}=0$ for all $i+1 \leq j \leq d$. Similarly, $\lim _{n \mapsto \omega} a \sigma_{n}$ is a positive (respectively, negative) constant if and only if $a_{i}=0$ for all $1 \leq i \leq d$ and $a_{0}>0$ (respectively, $a_{0}<0$ ). This allows us to translate the solvability of a limit problem into the satisfiability of an arithmetic formula.

Definition 5.23 (SMT Encoding of Limit Problems). Let $a$ be a polynomial arithmetic expression of degree $d$ and let $\sigma_{n}$ instantiate each occurring variable $x$ by $m_{x} \cdot n+k_{x}$ where $m_{x}, k_{x}$ are abstract coefficients. Let $a \sigma_{n}=a_{d} \cdot n^{d}+\cdots+a_{0} \cdot n^{0}$ where $a_{0}, \ldots, a_{d}$ do not contain $n$. We define

$$
\operatorname{smt}\left(a^{\bullet}\right)= \begin{cases}\bigvee_{i=1}^{d}\left(a_{i}>0 \wedge \bigwedge_{j=i+1}^{d} a_{j}=0\right) & \text { if } \bullet=+ \\ \bigvee_{i=1}^{d}\left(a_{i}<0 \wedge \bigwedge_{j=i+1}^{d} a_{j}=0\right) & \text { if } \bullet=- \\ \bigwedge_{j=1}^{d} a_{j}=0 \wedge a_{0}>0 & \text { if } \bullet=+! \\ \bigwedge_{j=1}^{d} a_{j}=0 \wedge a_{0}<0 & \text { if } \bullet=-! .\end{cases}
$$

We lift smt to limit problems $L$ where $a$ is a polynomial for each $a^{\bullet} \in L$ by defining $\operatorname{smt}(L)=$ $\bigwedge a^{\bullet} \in L \operatorname{smt}\left(a^{\bullet}\right)$.

Furthermore, given a polynomial cost $c$ of degree $d$ with $c \sigma_{n}=c_{d} \cdot n^{d}+\cdots+c_{0} \cdot n^{0}$ where $c_{0}, \ldots, c_{d}$ do not contain $n$, we define $\operatorname{smt}_{c, i}(L)=\operatorname{smt}(L) \wedge c_{i}>0$ for each $1 \leq i \leq d$. Finally, for the program variables $\bar{x}$, we define $\operatorname{smt}_{\omega}(L)=\operatorname{smt}(L) \wedge \wedge_{x \in \bar{x}} m_{x}=0$.

To solve a limit problem $L$, it suffices to find a solution for $\operatorname{smt}(L)$, because then the substitution that results from instantiating the abstract coefficients of $\sigma_{n}$ accordingly is a solution for the limit problem $L$. However, to maximize the cost $c$, one should try to find a solution for $\operatorname{smt}_{\omega}(L)$ or $\operatorname{smt}_{c, i}(L)$ where $i$ is as large as possible. The reason is that a solution for $\operatorname{smt}_{\omega}(L)$ allows us to deduce unbounded costs (provided that the initial limit problem contained $c^{+}$, i.e., the cost is nonconstant for each solution of $L$ ), as the corresponding substitution $\sigma_{n}$ maps all program variables $x$ to constants $k_{x}$ that do not depend on $n$. A solution for $\operatorname{smt}_{c, i}(S)$ allows us to prove a polynomial lower bound whose degree is at least $i$ via Theorem 5.5 and Lemma 5.8 (since $\left|\bar{x} \sigma_{n}\right| \in O(n)$ ).

Example 5.24 (Encoding the Initial Limit Problem for Program (19) Continued). We now show how to encode the initial limit problem $\left\{\left(\frac{1}{2} x^{2}+\frac{1}{2} x-1\right)^{+}\right\}$from Example 5.4. ${ }^{20}$ Since $x \sigma_{n}=m_{x} \cdot n+k_{x}$,

\footnotetext{
${ }^{20}$ For reasons of simplicity, we do not include the cost of the rule in the initial limit problem.
} 
we have $\left(\frac{1}{2} x^{2}+\frac{1}{2} x-1\right) \sigma_{n}=a_{2} \cdot n^{2}+a_{1} \cdot n+a_{0}$ with $a_{2}, a_{1}, a_{0}$ as in Example 5.22. Thus,

$$
\operatorname{smt}\left(\left\{\left(\frac{1}{2} x^{2}+\frac{1}{2} x-1\right)^{+}\right\}\right)=\left(a_{2}>0 \vee\left(a_{1}>0 \wedge a_{2}=0\right)\right) .
$$

Now SMT solvers can easily find a solution like, e.g., $\left\{m_{x} / 1, k_{x} / 0\right\}$.

Example 5.25 (Encoding the Initial Limit Problem for Example 5.1). Next, we encode the initial limit problem $\left\{\left(x-y^{2}\right)^{+!}, y^{+}\right\}$for Example 5.1. Since $x \sigma_{n}=m_{x} \cdot n+k_{x}$ and $y \sigma_{n}=m_{y} \cdot n+$ $k_{y}$, we have $\left(x-y^{2}\right) \sigma_{n}=m_{x} \cdot n+k_{x}-\left(m_{y} \cdot n+k_{y}\right)^{2}=-m_{y}^{2} \cdot n^{2}+\left(m_{x}-2 \cdot m_{y} \cdot k_{y}\right) \cdot n+k_{x}-$ $k_{y}^{2}$. Thus,

$$
\operatorname{smt}\left(\left\{\left(x-y^{2}\right)^{+!}, y^{+}\right\}\right)=\left(-m_{y}^{2}=0 \wedge m_{x}-2 \cdot m_{y} \cdot k_{y}=0 \wedge k_{x}-k_{y}^{2}>0 \wedge m_{y}>0\right) .
$$

Here, the first three (in)equations are the encoding of $\left(x-y^{2}\right)^{+!}$and the last inequation is the encoding of $y^{+}$. As $-m_{y}^{2}=0$ implies $m_{y}=0$, the overall formula is unsatisfiable. State-of-the-art SMT solvers can prove unsatisfiability of $\operatorname{smt}\left(\left\{\left(x-y^{2}\right)^{+}, y^{+}\right\}\right)$within milliseconds. This is not surprising, since we instantiated $x$ with a non-linear expression in Example 5.16 to find a solution, but Definition 5.23 instantiates $x$ with a linear template polynomial.

Thus, Example 5.25 shows that even if all arithmetic expressions in the analyzed limit problem are polynomials, $\rightsquigarrow$ is still required, i.e., our SMT-based technique does not subsume the calculus of Section 5.2. ${ }^{21}$ Note that the new SMT-based technique can be integrated into the calculus from Section 5.2 seamlessly. In other words, one can first simplify a limit problem with the transformation $\rightsquigarrow$ for a few steps and then apply the SMT-based technique to find a solution for the obtained limit problem. For instance, the initial limit problem $\left\{\left(x-y^{2}\right)^{+!}, y^{+}\right\}$in Example 5.25 can easily be solved via the SMT encoding from Definition 5.23 after applying the substitution $\left\{x / y^{2}+1\right\}$ as in Example 5.16.

The following theorem shows how a solution for the SMT problem $\operatorname{smt}(L)$ can be used to obtain a solution for the limit problem $L$.

Theorem 5.26 (Solving Limit Problems via SMT). Let L be a limit problem such that each expression in $L$ is a polynomial and let $\sigma$ be an integer substitution such that $\sigma=\operatorname{smt}(L)$. Then

$$
\sigma_{n} \circ \sigma=\left\{x /\left(m_{x} \sigma \cdot n+k_{x} \sigma\right) \mid x \in \mathcal{V}(L)\right\}
$$

is a solution for $L$.

Proof. First note that $\sigma_{n} \circ \sigma$ is clearly an integer substitution for each $n \in \mathbb{N}$. We have to show that $\lambda n . a \sigma_{n} \sigma$ satisfies $\bullet$ for any $a^{\bullet} \in L$. Let $d$ be the degree of $a$. Then, we have $a \sigma_{n}=a_{d} \cdot n^{d}+$ $\cdots+a_{0} \cdot n^{0}$ for suitable expressions $a_{0}, \ldots, a_{d}$ over $\left\{m_{x}, k_{x} \mid x \in \mathcal{V}(a)\right\}$ that do not contain $n$.

We first consider the case $\bullet=+$ (the case $\bullet=-$ works analogously). Then $\sigma=\operatorname{smt}(L)$ implies $\sigma \models \operatorname{smt}\left(a^{+}\right)$, i.e., $\sigma \mid=\bigvee_{i=1}^{d}\left(a_{i}>0 \wedge \bigwedge_{j=i+1}^{d} a_{j}=0\right)$. Hence, there exists an $1 \leq i \leq d$ with $a_{i} \sigma>0$ and we have

$$
a \sigma_{n} \sigma=a_{i} \sigma \cdot n^{i}+\cdots+a_{0} \sigma \cdot n^{0} .
$$

Thus, we obtain $\lim _{n \mapsto \omega} a \sigma_{n} \sigma=\omega$, i.e., $\lambda$ n. $a \sigma_{n} \sigma$ satisfies + .

Now, we consider the case $\bullet=+$ ! (the case $\bullet=-$ ! works analogously). Then $\sigma=\operatorname{smt}(L)$ implies $\sigma \mid=\operatorname{smt}\left(a^{+!}\right)$, i.e., $\sigma \mid=\bigwedge_{j=1}^{d} a_{j}=0 \wedge a_{0}>0$. Hence, we have

$$
\lim _{n \mapsto \omega} a_{n} \sigma_{n} \sigma=\lim _{n \mapsto \omega} a_{0} \sigma=a_{0} \sigma>0,
$$

i.e., $\lambda$ n. $a \sigma_{n} \sigma$ satisfies + !.

\footnotetext{
${ }^{21}$ We could also use non-linear template polynomials for $\sigma_{n}$, but in any case the degree of the template polynomials has to be fixed in advance and thus, it may be insufficient for the problem at hand.
}

ACM Transactions on Programming Languages and Systems, Vol. 42, No. 3, Article 13. Publication date: October 2020. 
Example 5.27 (Solving the Initial Limit Problem for Program (19)). In Example 5.24, we saw that $\sigma=\left\{m_{x} / 1, k_{x} / 0\right\} \mid=\operatorname{smt}\left(\left\{\left(\frac{1}{2} x^{2}+\frac{1}{2} x-1\right)^{+}\right\}\right)$. Hence, according to Theorem 5.26, $\sigma_{n} \circ \sigma=\left\{x /\left(m_{x}\right.\right.$. $\left.\left.n+k_{x}\right)\right\} \circ\left\{m_{x} / 1, k_{x} / 0\right\}$ with $x \sigma_{n} \sigma=n$ solves the limit problem $\left\{\left(\frac{1}{2} x^{2}+\frac{1}{2} x-1\right)^{+}\right\}$. This corresponds to the solution with the family of substitutions (35). As explained in Example 5.9, this proves $\operatorname{rc}_{\mathcal{P}}(n) \in \Omega\left(n^{4}\right)$ for the program $\mathcal{P}=\{(19)\}$ and hence, also for the program in Figure 1(b).

To integrate Theorem 5.26 into the calculus of Section 5.2, we proceed as follows: Whenever the current limit problem $L$ for a rule $\alpha$ only contains polynomial arithmetic expressions, one tries to find a solution for $\operatorname{smt}_{\omega}(L)$ or $\operatorname{smt}_{c, i}(L)$ where $i$ is initially set to the degree of the cost $c$ of the rule $\alpha$ and decremented until the SMT solver finds a solution. As soon as a solution is found, one can either return the resulting family of substitutions that solves $L$ or keep searching for a better solution. To this end, one can either backtrack or continue simplifying $L$ via $\rightsquigarrow$. Similarly, if the SMT solver does not find a solution one can either backtrack or keep simplifying $L$ via $\rightsquigarrow$.

Note that the intention of Theorem 5.26 and its integration into $\rightsquigarrow$ is not to add more power to $\rightsquigarrow$. Instead, as often as possible one should delegate the search for a solution to SMT solvers (which are very efficient in solving search problems) instead of relying on heuristics. This may, of course, also lead to better results in cases where the heuristics used for $\rightsquigarrow$ are not ideal. For example, consider a simplified rule with cost $x \cdot\left(1-t v^{2}\right)$ and guard $-2<t v<2$. The heuristic discussed at the end of Section 5.2 would instantiate $t v$ with the bounds implied by the guard, i.e., it would apply the substitution $\theta=\{t v / 1\}$ or $\theta=\{t v /-1\}$, resulting in the unsolvable limit problem $\left\{\left(x \cdot\left(1-t v^{2}\right)\right)^{+}\right\} \theta=\left\{0^{+}\right\}$. In contrast, our SMT encoding allows us to find the solution $\{t v / 0, x / n\}$, which results in a linear lower bound.

Example 5.28 (Example 4.12 Continued). For the simplified facSum program with the rule (32), we obtain the initial limit problem

$$
\left\{(x-1)^{+},\left(\frac{1}{2} x^{2}+\frac{3}{2} x-2\right)^{+}\right\} .
$$

Using $\sigma_{n}=\left\{x /\left(m_{x} \cdot n+k_{x}\right)\right\}$, its SMT encoding is

$$
m_{x}>0 \wedge\left(\left(m_{x} \cdot k_{x}+\frac{3}{2} m_{x}>0 \wedge \frac{1}{2} m_{x}^{2}=0\right) \vee \frac{1}{2} m_{x}^{2}>0\right) .
$$

An SMT solver can find a model like $\sigma=\left\{m_{x} / 1, k_{x} / 0\right\}$, for example. This results in the solution

$$
\sigma_{n} \circ \sigma=\left\{x /\left(m_{x} \sigma \cdot n+k_{x} \sigma\right)\right\}=\{x / n\} .
$$

Applying it to the cost $\frac{1}{2} x^{2}+\frac{3}{2} x-2$ yields $\frac{1}{2} n^{2}+\frac{3}{2} n-2 \in \Omega\left(n^{2}\right)$. By Theorem 5.5 , this proves $\operatorname{rc}_{\{(32)\}}\left(\left|x \sigma_{n} \sigma\right|\right)=\operatorname{rc}_{\{(32)\}}(n) \in \Omega\left(n^{2}\right)$. By the soundness of the program simplification in Sections 3 and 4, we obtain $\operatorname{rc} \mathcal{P}(n) \in \Omega\left(n^{2}\right)$ for the original integer program $\mathcal{P}$ from Example 4.9.

\section{EXPERIMENTS}

To evaluate the performance of our approach, we implemented it in the tool LoAT ("Loop Acceleration Tool") ${ }^{22}$ using the recurrence solver PURRS [8] and the SMT solver Z3 [21]. We evaluated LoAT on the 689 benchmark integer programs from the evaluation [49] of the tool KoAT [14], which infers upper runtime bounds for integer programs. On average, the ITSs in the collection [49] have a size of 23.4 rules. In addition, the results of running LoAT on the examples from this article can be found on the website [33].

Comparison with Upper Bound Provers. As we are not aware of any other tool to compute worstcase lower bounds for integer programs, we compared our results with the asymptotically smallest

\footnotetext{
${ }^{22}$ Initially, LoAT stood for "Lower Bounds Analysis Tool," but we renamed it to reflect that LoAT's loop acceleration techniques can be used for several purposes, see the approach [32].
} 
Table 1. Best Upper Bound vs. LoAT

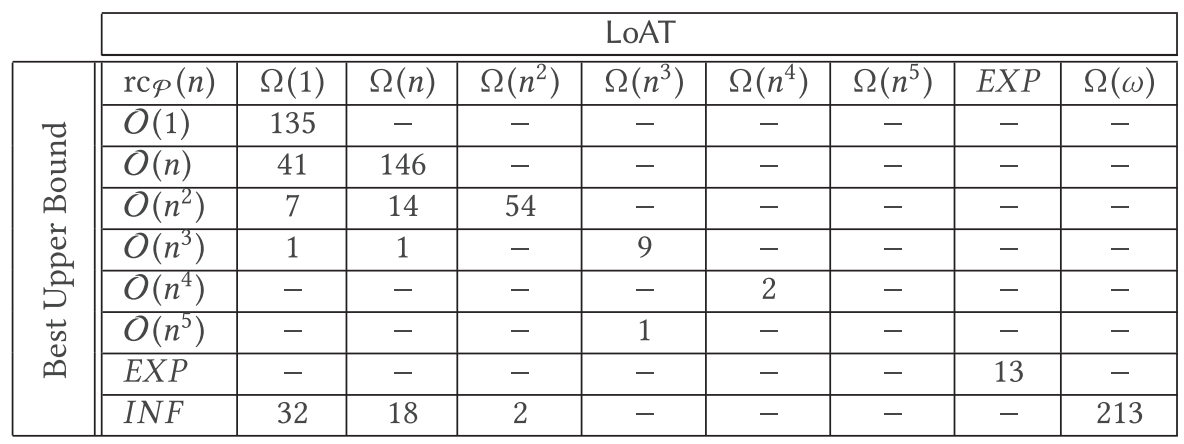

results of the tools KoAT, CoFloCo [26, 27], Loopus [57], and RanK [6], that compute upper runtime bounds for integer programs.

The results of running all tools on an Azure F4s v2 instance with a timeout of 60 seconds per example are shown in Table 1. LoAT inferred non-constant lower bounds for 473 examples. For 135 additional examples, the upper bound $\operatorname{rc} \mathcal{P}(n) \in O(1)$ was proved and thus, the lower bound $\Omega(1)$ inferred by LoAT is optimal. Thus, LoAT finds non-trivial or optimal bounds on $473+$ $135=608(88.2 \%)$ of all examples. For 572 examples $(83.0 \%)$, the inferred bounds are asymptotically tight (e.g., lower and upper bounds coincide). Whenever an exponential upper bound was proved, LoAT also proved an exponential lower bound (i.e., $\operatorname{rc} \mathcal{P}(n) \in \Omega\left(k^{n}\right)$ for some $k>1$ ). It proved the existence of executions with unbounded length in 213 cases (this includes both non-terminating examples and examples whose runtime depends on temporary variables). On average, LoAT required 2 seconds per example. For 10 of the 689 examples, LoAT could not finish its analysis due to the timeout. The reason for most timeouts is that the number of rules gets too large during the program simplification and hence, LoAT fails to compute a simplified program in time.

One reason why the bounds inferred by LoAT do not always coincide with the upper bounds obtained by other tools may of course be that these upper bounds are not necessarily tight. If the lower bound is too small, then in our experience the most common reasons for imprecision are the following: For some loops, LoAT fails to find (non-trivial) metering functions. But if LoAT finds a metering function, then the precision of the metering function is usually very good. Another reason for imprecision are unsuitable instantiations of temporary variables. Finally, LoAT heuristically deletes rules from the analyzed program if the number of rules becomes too large, which is another common source of imprecision. Nevertheless, our experiments show that optimal lower bounds are inferred for the large majority of examples.

Evaluating Individual Contributions and Comparison with Best-case Lower Bounds. In a second experiment, we performed an individual evaluation of the main contributions of this article that are new compared to our earlier paper [29]. As baseline, we took LoAT-Basic, the version of LoAT implementing the techniques in our earlier work [29]. ${ }^{23}$ Using LoAT-Basic as a starting point, we considered the following four variants:

- LoAT-Cond adds support for conditional metering functions, as introduced in Theorem 3.4.

- LoAT-Rec is LoAT-Basic extended by the handling of non-tail-recursive programs (described in Section 4).

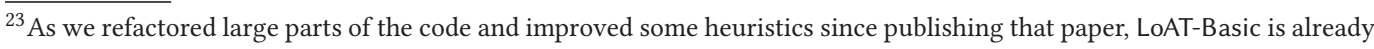
more powerful than the version of LoAT from 2016 that we used in the paper [29].
} 
Table 2. Evaluating Individual Contributions in LoAT and Comparison with Best-case Lower Bounds

\begin{tabular}{|l||r|r|r|r|r|r|r|r|}
\hline Tool & $\Omega(1)$ & $\Omega(n)$ & $\Omega\left(n^{2}\right)$ & $\Omega\left(n^{3}\right)$ & $\Omega\left(n^{4}\right)$ & $E X P$ & $\Omega(\omega)$ & time (s) \\
\hline \hline LoAT-Basic & 269 & 159 & 42 & 2 & 2 & 5 & 210 & 2.29 \\
\hline LoAT-Cond & 268 & 159 & 43 & 3 & 2 & 5 & 209 & 2.32 \\
\hline LoAT-Rec & 227 & 175 & 55 & 6 & 2 & 13 & 211 & 2.44 \\
\hline LoAT-SMT & 263 & 161 & 43 & 2 & 2 & 5 & 213 & 1.68 \\
\hline LoAT-Just-SMT & 263 & 161 & 43 & 2 & 2 & 5 & 213 & 1.64 \\
\hline LoAT & 216 & 179 & 56 & 10 & 2 & 13 & 213 & 1.91 \\
\hline \hline CoFloCo & 454 & 189 & 40 & 6 & 0 & 0 & 0 & 2.00 \\
\hline
\end{tabular}

- LoAT-SMT is like LoAT-Basic, but it applies the SMT encoding of limit problems from Section 5.3 in addition to the calculus for the transformation of limit problems that is used in LoAT-Basic. For this combination, we use a strategy that first simplifies limit problems with the calculus if the guard or the cost of the analyzed simplified rule contains non-polynomial arithmetic. However, the SMT encoding is used whenever it is applicable. Whenever there was a choice during the application of the calculus, we backtrack afterwards and apply the calculus again to examine the remaining possibilities. The analysis of the rule terminates as soon as LoAT proves a bound that is asymptotically equal to its cost function, when the timeout specified by the user expires, or when there are no further possibilities to backtrack (where we use suitable heuristics to ensure that case (C) of our calculus is only applied with finitely many substitutions). Then the largest bound found so far is returned as the result.

- LoAT-Just-SMT is like LoAT-SMT, but in contrast to LoAT-SMT, LoAT-Just-SMT never applies the calculus again once the SMT encoding is applicable.

Note that each of these variants only adds one single new contribution to LoAT-Basic, whereas the other new contributions are disabled. The intention of the last variant is to compare the power and performance of the calculus from Section 5.2 with the SMT encoding of Section 5.3 (whereas LoAT-SMT represents the combination of both techniques). However, as the novel SMT encoding only applies to polynomial limit problems, LoAT-Just-SMT still uses the calculus from Section 5.2 for non-polynomial limit problems.

The results of our experiments are summarized in Table 2: LoAT-Basic already uses an optimization from our paper [29] that is similar to (but weaker than) the conditional metering functions of Section 3.1. Conditional metering functions do not only improve the formalization and presentation of our approach (by integrating the optimization of the paper [29] into our concept of metering functions), but they also lead to a minimal change in power: The detailed experimental results on our website [33] show that LoAT-Cond deduces better (i.e., larger) asymptotic bounds in eight cases, whereas LoAT-Basic deduces better asymptotic bounds in four cases. Adding support for arbitrary recursion allows LoAT to infer non-constant bounds for 41 of the 50 non-tailrecursive examples in the collection (where a constant upper bound was proved for three of these examples). The full version of LoAT even obtains non-constant bounds for 44 of these examples. The SMT encoding of limit problems improves the performance compared to LoAT-Basic by $26 \%$. Moreover, LoAT-SMT deduces a better asymptotic bound than LoAT-Basic in eight cases, whereas LoAT-Basic infers a better asymptotic bound in one case. The configurations LoAT-SMT and LoAT-Just-SMT yield identical results. So when disregarding limit problems with non-polynomial arithmetic, the calculus from Section 5.2 is outperformed by the novel SMT encoding from 
Section 5.3 in our experiments on the examples from the collection [49]. However, this is not true in general, as shown by Example 5.25. Moreover, the calculus from Section 5.2 is still required for the analysis of limit problems with non-polynomial arithmetic, i.e., for all examples where LoAT proves exponential lower bounds. In LoAT (i.e., in the last line of Table 2), we extended LoAT-Basic by all new contributions. This results in a significant improvement in both power and runtime.

Finally, in the last row of Table 2, we used the tool CoFloCo $[26,27]$ to compute best-case lower bounds. While the asymptotic bounds obtained from LoAT and CoFloCo coincide for 335 of the 689 examples, the results of the two tools are of course not directly comparable, since LoAT infers worst-case lower bounds, but in general, a worst-case lower bound is not a valid best-case lower bound. Moreover, CoFloCo analyzes different program paths (so-called chains) separately and infers individual lower bounds for them. However, similar to the experimental evaluation of CoFloCo [27], the results in the last row of Table 2 do not take the preconditions of the different chains into account, but they are simply the maximum lower bound of all chains. Thus, they are not always asymptotic best-case lower bounds for the whole program. So the purpose of the last row is only to indicate that LoAT's performance is also convincing when comparing it to the performance of other tools for the inference of (other forms of) lower bounds.

For a detailed experimental evaluation of our implementation as well as a pre-compiled binary of LoAT, we refer to the website [33]. The source code of LoAT is freely available [52].

\section{RELATED WORK}

While there are many techniques to infer upper bounds on the worst-case complexity of integer programs [1-8, 10, 14, 19, 20, 26, 27, 39, 42, 45-47, 57, 58], there is little work on lower bounds. In the paper [7], it is briefly mentioned that their technique could also be adapted to infer lower instead of upper bounds for abstract cost rules, i.e., integer procedures with (possibly multiple) outputs. However, this only considers best-case lower bounds instead of worst-case lower bounds, as in our technique. Upper and lower bounds for cost relations are inferred in the approaches [4, 27]. Cost relations extend recurrence equations such that, e.g., non-determinism can be modeled. However, this technique also considers best-case lower bounds only. A method for best-case lower bounds for logic programs is presented in the work [22].

Note that techniques to infer lower bounds on the best-case complexity differ fundamentally from techniques for the inference of worst-case lower bounds. To deduce best-case lower bounds, one has to prove that a certain bound holds for every program run. Thus, as in the case of worst-case upper bounds, over-approximating techniques are used to ensure that the proven bound covers all program runs, i.e., even though such techniques under-approximate the runtime of the program, they over-approximate the set of all program runs.

In contrast, techniques to infer lower bounds on the worst-case complexity have to identify families of inputs (i.e., witnesses) that result in expensive program runs. Thus, for the inference of worst-case lower bounds, over-approximations are usually unsound, since one has to ensure that the witness of the proven lower bound corresponds to "real" program runs. Thus, underapproximating techniques have to be used to infer lower bounds on the worst-case complexity.

Nevertheless, our approach has certain aspects in common with the technique [4], that also uses recurrence solving to compute a closed form for the costs of several consecutive applications of a cost equation with direct recursion, which corresponds to a simple loop or simple recursion in our setting. However, as mentioned above, the analyses for best-case lower bounds [4, 27] have to reason about all program runs. Thus, there the handling of non-determinism is challenging, as all possible non-deterministic choices have to be taken into account. In contrast, we can treat temporary variables (which we use to model non-determinism) as constants when computing the iterated update and cost. Thus, our iterated update and cost only represent evaluations where 
temporary variables are instantiated with the same values in each iteration. This restriction is sound in our setting, as we only need to prove the existence of a certain family of program runs, i.e., we do not have to reason about all program runs. To reason about evaluations where the valuation of the temporary variables changes, we can instantiate them with expressions containing program variables via Instantiation (Theorem 3.12).

Since our computation of the iterated update relies on the existence of a single deterministic update, it is not applicable to simple recursions, which also prevents us from computing iterated costs when accelerating non-tail-recursive rules in Theorem 4.5. Thus, our handling of simple recursions may be improved by incorporating ideas from the approaches [4, 27] for the inference of bounds of cost equations with multiple recursive calls.

In earlier work [30], we introduced two techniques to infer worst-case lower bounds for term rewrite systems (TRSs). However, TRSs differ substantially from the programs considered here, since they do not allow integers and have no notion of a "program start." Thus, the techniques from our paper [30] are very different to the present article.

In contrast to the techniques for the computation of symbolic runtime bounds, the technique [15] generates test-cases that trigger the worst-case execution time of programs. The idea is to execute the program for small inputs, observe the required runtime, and then generalize those inputs that lead to expensive runs. In this way, one obtains generators, which can be used to construct larger inputs that presumably result in expensive runs as well. In contrast to the technique presented in the current article, the approach [15] operates on Java, i.e., it also supports data structures. However, the approach [15] does not try to infer symbolic bounds, which is the main purpose of our technique. Nevertheless, ideas from the approach [15] could be integrated into our framework. For example, a similar approach could be used to apply Instantiation (Theorem 3.12) in a way that leads to expensive runs.

The approach [60] can synthesize worst-case inputs, but the size of the input needs to be fixed a priori. This approach is fundamentally different from our technique to deduce symbolic worstcase bounds. However, the inputs that are synthesized by the technique [60] are provably optimal, whereas our worst-case lower bounds are correct, but not necessarily tight.

Inferring bounds on the runtime of programs has also been investigated for probabilistic programs. While there exist several approaches to find upper bounds on the expected runtime of such programs, again there are only very few works that consider the inference of lower bounds on the expected runtime of probabilistic programs [34, 36, 40, 54].

To simplify programs, we use Loop Acceleration to summarize the effect of applying a simple loop (or a simple recursion) repeatedly. Acceleration is mostly used in over-approximating settings [25, $38,48,53,59]$, where handling non-determinism is challenging, as loop summaries have to cover all possible non-deterministic choices. However, our technique is under-approximating, i.e., we can instantiate non-deterministic values arbitrarily.

The under-approximating acceleration technique [50] uses quantifier elimination, whereas our acceleration technique relies on metering functions. In our approach [32], we generalized the loop acceleration technique [50] to prove non-termination of integer programs. In future work, we will examine whether ideas from the techniques $[32,50]$ can also be incorporated into our framework for the inference of lower runtime bounds.

Another related approach [11, 12, 35] accelerates loops whose transitive closure can be expressed in Presburger Arithmetic. In particular, this is the case for loops whose transition relation can be described by octagons, i.e., conjunctions of inequations of the form $\pm x \pm y \leq c$ where $x, y$ are variables and $c \in \mathbb{Z}$, and for loops with Presburger-definable guards and affine updates $\mu(\bar{x})=A \cdot \bar{x}+\bar{c}$ with the finite monoid property, i.e., where the set $\left\{A^{i} \mid i \in \mathbb{N}\right\}$ is finite. In contrast, our acceleration technique does not necessarily compute the transitive closure 
of loops exactly. The reason is that our metering functions may be imprecise and that we approximate non-determinism by assuming that the values of temporary variables remain unchanged across loop iterations. However, our approach can also handle loops where the transitive closure cannot be expressed in Presburger Arithmetic.

The technique [1] infers asymptotic bounds from concrete bounds with a so-called context constraint $\varphi$, i.e., bounds of the form $\varphi \Longrightarrow r t \leq e$ or $\varphi \Longrightarrow r t \geq e$. Here, $r t$ is the runtime of the program and $e$ is a cost expression. These expressions are orthogonal to the expressions that are supported by our technique from Section 5 (e.g., $e$ may contain maximum and logarithm, but negative numbers are only allowed in sub-expressions of the form $\max (0, \ldots))$. The approach $[1]$ infers multi-variate asymptotic bounds, whereas our technique infers univariate bounds that are only parameterized in the size of the input. Moreover, the approach [1] does not aim to eliminate the context constraint, i.e., the resulting asymptotic bounds are of the form $\varphi \Longrightarrow r t \in O(b)$ or $\varphi \Longrightarrow r t \in \Omega(b)$. In contrast, eliminating the context constraints $\varphi$ is one of the main motivations for our technique to deduce asymptotic bounds from concrete bounds.

Our SMT encoding for limit problems from Section 5.3 inspired parts of our work [31], where we used a similar encoding to prove that termination is decidable for a certain class of integer loops. As in Section 5.3, the underlying idea of the approach [31] is to abstract the loop guard by focusing on its behavior "for large enough values of $n$." In the present work, the variable $n$ is the parameter of the family of substitutions that solves the limit problem. In the approach [31], $n$ represents the number of loop iterations. Due to the restricted form of the loops in the approach [31], their SMT encoding only requires linear integer arithmetic, such that we obtain a decision procedure for termination.

Finally, our concept of metering functions has been adapted to synthesize invariants [17]. Note that invariant inference and complexity analysis are closely related: Invariant inference techniques can be used to compute complexity bounds by introducing an additional counter that is incremented in each step and deducing an invariant that bounds its value [57]. Conversely, complexity analysis techniques can be used to bound the value of any arithmetic expression $b$ (i.e., to compute invariants) by choosing the costs of transitions in a way that reflects changes of the value of $b[55$, 57].

\section{CONCLUSION AND FUTURE WORK}

We introduced the first technique to infer lower bounds on the worst-case runtime complexity of integer programs, based on a modular program simplification framework. The main simplification techniques are Loop Acceleration and Recursion Acceleration, which rely on recurrence solving and metering functions, an adaptation of classical ranking functions. By eliminating loops and function symbols via Chaining and Partial Deletion, we eventually obtain simplified programs. We presented a technique to infer asymptotic lower bounds from simplified programs, which can also be used to find program vulnerabilities. An experimental evaluation with our tool LoAT demonstrates the applicability of our technique in practice [33,52].

In comparison to the preliminary version of our paper [29], we showed how to deduce conditional metering functions, we improved our program simplification by eliminating variables from the program, we extended our approach to non-tail-recursive programs, and we improved our technique to infer asymptotic lower bounds for simplified programs by an SMT encoding. (See Section 1 for a full list of the contributions of the current article compared to our earlier paper [29]).

There are several interesting directions for future work. First, one could couple LoAT with invariant inference techniques to improve its power. Furthermore, LoAT's heuristics to apply Instantiation are relatively simple and should be improved, e.g., by incorporating ideas from the 
technique [15]. Another interesting question is to what extent LoAT can benefit from more sophisticated techniques to infer metering functions. Possibilities include the inference of logarithmic or super-linear polynomial metering functions, but one could also adapt quasi-ranking functions [51] to our setting. Apart from that, we plan to investigate if our approach can benefit from alternative loop acceleration techniques [12, 32, 50]. Moreover, as mentioned in Section 7, ideas from the techniques $[4,27]$ could be adapted to under-approximate the costs of repeatedly applying simple recursions more precisely when accelerating them. Finally, one could generalize our program model to improve its expressiveness. In particular, one could consider the return values of auxiliary function calls (by allowing terms with nested occurrences of functions). Moreover, one could combine our technique with ideas from our approach [30] for the inference of lower bounds for term rewrite systems (i.e., programs operating on tree-shaped data structures) to analyze programs whose complexity depends on both integers and data structures.

\section{APPENDIX}

\section{A PROOF OF LEMMA 5.8}

Lemma 5.8 is based on Lemma 24 from our paper [30]. However, since the lemmas are slightly different and the proof for part (b) was omitted from the paper [30], we provide the proof of Lemma 5.8 in this Appendix. Moreover, part (c) of the lemma was not present in our earlier paper [30].

Lemma 5.8 (Bounds for Function Composition). Let $f: \mathbb{N} \rightarrow \mathbb{R}_{\geq 0} \cup\{\omega\}$ and $g: \mathbb{N} \rightarrow \mathbb{N}$ where $g(n) \in O\left(n^{d}\right)$ for some $d \in \mathbb{N}$ with $d>0$. Moreover, let $f(n)$ be weakly monotonically increasing for large enough $n$.

(a) If $g(n)$ is strictly monotonically increasing for large enough $n$ and $f(g(n)) \in \Omega\left(n^{k}\right)$ with $k \in \mathbb{N}$, then $f(n) \in \Omega\left(n^{\frac{k}{d}}\right)$.

(b) If $g(n)$ is strictly monotonically increasing for large enough $n$ and $f(g(n)) \in \Omega\left(k^{n}\right)$ with $k>1$, then $f(n) \in \Omega\left(e^{\sqrt[d]{n}}\right)$ for some number $e \in \mathbb{R}$ with $e>1$.

(c) If $g(n) \in O(1)$ and $f(g(n)) \notin O(1)$, then $f(n) \in \Omega(\omega)$.

Proof. For any $n_{0} \in \mathbb{N}$, let $\mathbb{N}_{\geq n_{0}}=\left\{n \in \mathbb{N} \mid n \geq n_{0}\right\}$. For any (total) function $h: M \rightarrow \mathbb{N}_{\geq n_{0}}$ with $M \subseteq \mathbb{N}$ where $M$ is infinite, we define $\lfloor h\rfloor(n): \mathbb{N}_{\geq \min (M)} \rightarrow \mathbb{N}_{\geq n_{0}}$ and $\lceil h\rceil(n): \mathbb{N} \rightarrow \mathbb{N}_{\geq n_{0}}$ by:

$$
\begin{aligned}
& \lfloor h\rfloor(n)=h(\max \{x \in M \mid x \leq n\}), \\
& \lceil h\rceil(n)=h(\min \{x \in M \mid x \geq n\}) .
\end{aligned}
$$

Note that infinity of $h$ 's domain $M$ ensures that there is always an $x \in M$ with $x \geq n$. Since $\lfloor h\rfloor$ is only defined on $\mathbb{N}_{\geq \min (M)}$, there is always an $x \in M$ with $x \leq n$ for any $n \in \mathbb{N}_{\geq \min (M)}$.

To prove the lemma, as in the proof of Lemma 24 in our paper [30], we first show that if $h$ : $M \rightarrow \mathbb{N}_{\geq n_{0}}$ is strictly monotonically increasing and surjective, then

$$
\lfloor h\rfloor(n) \in\{\lceil h\rceil(n),\lceil h\rceil(n)-1\} \text { for all } n \in \mathbb{N}_{\geq \min (M)} .
$$

Afterwards, we prove (a)-(c) separately.

Claim 1. (38) holds, i.e., $\lfloor h\rfloor(n) \in\{\lceil h\rceil(n),\lceil h\rceil(n)-1\}$

To prove (38), let $n \in \mathbb{N}_{\geq \min (M)}$. If $n \in M$, then clearly $\lfloor h\rfloor(n)=\lceil h\rceil(n)$. If $n \notin M$, then let $\check{n}=$ $\max \{x \in M \mid x<n\}$ and $\hat{n}=\min \{x \in M \mid x>n\}$. Thus, $\check{n}<n<\hat{n}$. Strict monotonicity of $h$ implies $h(\check{n})<h(\hat{n})$. Assume that $h(\hat{n})-h(\check{n})>1$. Then by surjectivity of $h$, there is an $\bar{n} \in M$ with $h(\bar{n})=h(\check{n})+1$ and thus $h(\check{n})<h(\bar{n})<h(\hat{n})$. By strict monotonicity of $h$, we obtain $\check{n}<\bar{n}<\hat{n}$. 
Since $n \notin M$ and $\bar{n} \in M$ implies $n \neq \bar{n}$, we either have $\bar{n}<n$, which contradicts $\check{n}=\max \{\check{n} \in M \mid$ $\check{n}<n\}$ or $\bar{n}>n$, which contradicts $\hat{n}=\min \{\hat{n} \in M \mid \hat{n}>n\}$. Hence, $\lfloor h\rfloor(n)=h(\check{n})=h(\hat{n})-1=$ $\lceil h\rceil(n)-1$, which proves (38).

Claim 2. Lemma 5.8(a) holds, i.e., $f(g(n)) \in \Omega\left(n^{k}\right)$ implies $f(n) \in \Omega\left(n^{\frac{k}{d}}\right)$

For the proof of this claim, we slightly adapt the corresponding proof of Lemma 24 in our paper [30] to arbitrary functions $f$ and $g$. Note that $g(n) \in O\left(n^{d}\right)$ and $f(g(n)) \in \Omega\left(n^{k}\right)$ imply

$$
\exists n_{0}, m, m^{\prime}>0 . \forall n \in \mathbb{N}_{\geq n_{0}} \cdot g(n) \leq m \cdot n^{d} \wedge m^{\prime} \cdot n^{k} \leq f(g(n)) .
$$

We can choose $n_{0}$ large enough such that $\left.f\right|_{\mathbb{N}_{\geq n_{0}}}$ is weakly and $\left.g\right|_{\mathbb{N}_{\geq n_{0}}}$ is strictly monotonically increasing, where for any function $h: \mathbb{N} \rightarrow \mathbb{N}$ and any $M \subseteq \mathbb{N},\left.h\right|_{M}$ denotes the restriction of $h$ to $M$. Let $M=\left\{g(n) \mid n \geq n_{0}\right\}$ and let $g^{-1}: M \rightarrow \mathbb{N}_{\geq n_{0}}$ be the function such that $g\left(g^{-1}(n)\right)=n$. Note that $g^{-1}$ exists, since strict monotonicity of $g$ implies injectivity of $g$. By instantiating $n$ with $g^{-1}(n)$, we obtain

$$
\exists n_{0}, m, m^{\prime}>0 . \forall n \in M . g\left(g^{-1}(n)\right) \leq m \cdot\left(g^{-1}(n)\right)^{d} \wedge m^{\prime} \cdot\left(g^{-1}(n)\right)^{k} \leq f\left(g\left(g^{-1}(n)\right)\right),
$$

which simplifies to

$$
\exists n_{0}, m, m^{\prime}>0 . \forall n \in M . \quad n \leq m \cdot\left(g^{-1}(n)\right)^{d} \wedge m^{\prime} \cdot\left(g^{-1}(n)\right)^{k} \leq f(n) .
$$

When dividing by $m$ and taking the $d$ th root on both sides of the first inequation, we get

$$
\exists n_{0}, m, m^{\prime}>0 . \forall n \in M . \quad \sqrt[d]{\frac{n}{m}} \leq g^{-1}(n) \wedge m^{\prime} \cdot\left(g^{-1}(n)\right)^{k} \leq f(n) .
$$

By monotonicity of $\sqrt[d]{\frac{n}{m}}$ and $f(n)$ in $n$, this implies

$$
\exists n_{0}, m, m^{\prime}>0 . \forall n \in \mathbb{N}_{\geq g\left(n_{0}\right)} \cdot \sqrt[d]{\frac{n}{m}} \leq\left\lceil g^{-1}\right\rceil(n) \wedge m^{\prime} \cdot\left(\left\lfloor g^{-1}\right\rfloor(n)\right)^{k} \leq f(n) .
$$

Note that $\left.g\right|_{\mathbb{N}_{\geq n_{0}}}$ is total and hence, $g^{-1}: M \rightarrow \mathbb{N}_{\geq n_{0}}$ is surjective. Moreover, by strict monotonicity of $\left.g\right|_{\mathbb{N}_{\geq n_{0}}}, M$ is infinite and $g^{-1}$ is also strictly monotonically increasing. Hence, by (38), we get $\left\lceil g^{-1}\right\rceil(n) \leq\left\lfloor g^{-1}\right\rfloor(n)+1$ for all $n \in \mathbb{N}_{\geq g\left(n_{0}\right)}$. Thus,

$$
\exists n_{0}, m, m^{\prime}>0 . \forall n \in \mathbb{N}_{\geq g\left(n_{0}\right)} \cdot \sqrt[d]{\frac{n}{m}}-1 \leq\left\lfloor g^{-1}\right\rfloor(n) \wedge m^{\prime} \cdot\left(\left\lfloor g^{-1}\right\rfloor(n)\right)^{k} \leq f(n),
$$

which implies

$$
\exists n_{0}, m, m^{\prime}>0 . \forall n \in \mathbb{N}_{\geq g\left(n_{0}\right)} \cdot m^{\prime} \cdot\left(\sqrt[d]{\frac{n}{m}}-1\right)^{k} \leq f(n) .
$$

Therefore, $\exists m>0 . f(n) \in \Omega\left(\left(\sqrt[d]{\frac{n}{m}}-1\right)^{k}\right)$ and thus, $f(n) \in \Omega\left(n^{\frac{k}{d}}\right)$.

Claim 3. Lemma 5.8(b) holds, i.e., $f(g(n)) \in \Omega\left(k^{n}\right)$ implies $f(n) \in \Omega\left(e^{\sqrt[d]{n}}\right)$ for some $e>1$

The proof is analogous to the proof of the case $f(g(n)) \in \Omega\left(n^{k}\right)$, but it was not given in the paper [30]. Here, $g(n) \in O\left(n^{d}\right)$ and $f(g(n)) \in \Omega\left(k^{n}\right)$ imply

$$
\exists n_{0}, m, m^{\prime}>0 . \forall n \in \mathbb{N}_{\geq n_{0}} \cdot g(n) \leq m \cdot n^{d} \wedge m^{\prime} \cdot k^{n} \leq f(g(n)) .
$$

Again, we can choose $n_{0}$ large enough such that $\left.f\right|_{\mathbb{N}_{\geq n_{0}}}$ is weakly and $\left.g\right|_{\mathbb{N}_{\geq n_{0}}}$ is strictly monotonically increasing. As in the proof of the previous claim, let $M=\left\{g(n) \mid n \geq n_{0}\right\}$ and let $g^{-1}: M \rightarrow$ $\mathbb{N}_{\geq n_{0}}$ be the function such that $g\left(g^{-1}(n)\right)=n$. By instantiating $n$ with $g^{-1}(n)$, we obtain

$$
\exists n_{0}, m, m^{\prime}>0 . \forall n \in M . g\left(g^{-1}(n)\right) \leq m \cdot\left(g^{-1}(n)\right)^{d} \wedge m^{\prime} \cdot k^{g^{-1}(n)} \leq f\left(g\left(g^{-1}(n)\right)\right),
$$


which simplifies to

$$
\exists n_{0}, m, m^{\prime}>0 . \forall n \in M . \quad n \leq m \cdot\left(g^{-1}(n)\right)^{d} \wedge m^{\prime} \cdot k^{g^{-1}(n)} \leq f(n) .
$$

When dividing by $m$ and taking the $d$ th root on both sides of the first inequation, we get

$$
\exists n_{0}, m, m^{\prime}>0 . \forall n \in M . \quad \sqrt[d]{\frac{n}{m}} \leq g^{-1}(n) \wedge m^{\prime} \cdot k^{g^{-1}(n)} \leq f(n) .
$$

By monotonicity of $\sqrt[d]{\frac{n}{m}}$ and $f(n)$ in $n$, this implies

$$
\exists n_{0}, m, m^{\prime}>0 . \forall n \in \mathbb{N}_{\geq g\left(n_{0}\right)} \cdot \sqrt[d]{\frac{n}{m}} \leq\left\lceil g^{-1}\right\rceil(n) \wedge m^{\prime} \cdot k^{\left\lfloor g^{-1}\right\rfloor(n)} \leq f(n) .
$$

As in the proof of Claim 2, $\left.g\right|_{\mathbb{N}_{\geq n_{0}}}$ is total and hence, $g^{-1}: M \rightarrow \mathbb{N}_{\geq n_{0}}$ is surjective. Moreover, by strict monotonicity of $\left.g\right|_{\mathbb{N}_{\geq n_{0}}}, M$ is infinite and $g^{-1}$ is also strictly monotonically increasing. Hence, by (38), we get $\left\lceil g^{-1}\right\rceil(n) \leq\left\lfloor g^{-1}\right\rfloor(n)+1$ for all $n \in \mathbb{N}_{\geq g\left(n_{0}\right)}$. Thus,

$$
\exists n_{0}, m, m^{\prime}>0 . \forall n \in \mathbb{N}_{\geq g\left(n_{0}\right)} \cdot \sqrt[d]{\frac{n}{m}}-1 \leq\left\lfloor g^{-1}\right\rfloor(n) \wedge m^{\prime} \cdot k^{\left\lfloor g^{-1}\right\rfloor(n)} \leq f(n) .
$$

Since $k>1$, this implies

$$
\exists n_{0}, m, m^{\prime}>0 . \forall n \in \mathbb{N}_{\geq g\left(n_{0}\right)} \cdot m^{\prime} \cdot k^{\sqrt[d]{\frac{n}{m}}-1} \leq f(n),
$$

which is equivalent to

$$
\exists n_{0}, m, m^{\prime}>0 . \forall n \in \mathbb{N}_{\geq g\left(n_{0}\right)} \cdot \frac{m^{\prime}}{k} \cdot k \sqrt[d]{\frac{n}{m}} \leq f(n) .
$$

Since $m^{\prime}>0$ and $k>1$, we have $m^{\prime \prime}=\frac{m^{\prime}}{k}>0$ and thus, we get

$$
\exists n_{0}, m, m^{\prime \prime}>0 . \forall n \in \mathbb{N}_{\geq g\left(n_{0}\right)} \cdot m^{\prime \prime} \cdot k^{\sqrt[d]{\frac{n}{m}}} \leq f(n),
$$

which is equivalent to

$$
\exists n_{0}, m, m^{\prime \prime}>0 . \forall n \in \mathbb{N}_{\geq g\left(n_{0}\right)} \cdot m^{\prime \prime} \cdot k^{\frac{d \sqrt{n}}{\sqrt{m}}} \leq f(n) .
$$

Since $m>0$, we have $r=\sqrt[d]{m}>0$ and hence:

$$
\exists n_{0}, r, m^{\prime \prime}>0 . \forall n \in \mathbb{N}_{\geq g\left(n_{0}\right)} \cdot m^{\prime \prime} \cdot k^{\frac{d \sqrt{n}}{r}} \leq f(n),
$$

which is equivalent to

$$
\exists n_{0}, r, m^{\prime \prime}>0 . \forall n \in \mathbb{N}_{\geq g\left(n_{0}\right)} \cdot m^{\prime \prime} \cdot \sqrt[r]{k} \sqrt[d]{n} \leq f(n) .
$$

Finally, $k>1$ implies $e=\sqrt[r]{k}>1$ and we obtain

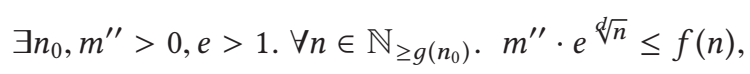

which implies

$$
\exists e>1 . f(n) \in \Omega\left(e^{\sqrt[d]{n}}\right) .
$$

Claim 4. Lemma 5.8(c) holds, i.e., $g(n) \in O(1)$ and $f(g(n)) \notin O(1)$ implies $f(n) \in \Omega(\omega)$

Note that $f(g(n)) \notin O(1)$ means that

$$
\forall m \in \mathbb{N} . \exists n \in \mathbb{N} . \quad f(g(n))>m .
$$

By $g(n) \in O(1)$, we have

$$
\exists m^{\prime} \in \mathbb{N} . \forall n \in \mathbb{N} . g(n) \leq m^{\prime} .
$$


As $f$ is weakly monotonic, we have $f(g(n)) \leq f\left(m^{\prime}\right)$ for all $n$. Hence, we get

$$
\exists m^{\prime} \in \mathbb{N} . \forall m \in \mathbb{N} . f\left(m^{\prime}\right)>m .
$$

This implies that $f\left(m^{\prime}\right)=\omega$. By weak monotonicity of $f$, we obtain

$$
\exists m^{\prime} \in \mathbb{N} . \forall n \geq m^{\prime} . f(n)=\omega .
$$

which means $f(n) \in \Omega(\omega)$.

\section{ACKNOWLEDGMENTS}

We thank Samir Genaim, Jan Böker, and Jera Hensel for discussions and comments. We are also very grateful to the anonymous reviewers for many helpful suggestions.

\section{REFERENCES}

[1] Elvira Albert, Diego E. Alonso-Blas, Puri Arenas, Samir Genaim, and Germán Puebla. 2009. Asymptotic resource usage bounds. In APLAS'09 (LNCS 5904). 294-310.

[2] Elvira Albert, Puri Arenas, Samir Genaim, and Germán Puebla. 2011. Closed-form upper bounds in static cost analysis. 7. Autom. Reas. 46, 2 (2011), 161-203.

[3] Elvira Albert, Puri Arenas, Samir Genaim, Germán Puebla, and Damiano Zanardini. 2012. Cost analysis of objectoriented bytecode programs. Theor. Comput. Sci. 413, 1 (2012), 142-159.

[4] Elvira Albert, Samir Genaim, and Abu N. Masud. 2013. On the inference of resource usage upper and lower bounds. ACM Trans. Comput. Log. 14, 3 (2013), 22:1-22:35.

[5] Elvira Albert, Miquel Bofill, Cristina Borralleras, Enrique Martin-Martin, and Albert Rubio. 2019. Resource analysis driven by (conditional) termination proofs. Theor. Pract. Log. Prog. 19, 5-6 (2019), 722-739.

[6] Christophe Alias, Alain Darte, Paul Feautrier, and Laure Gonnord. 2010. Multi-dimensional rankings, program termination, and complexity bounds of flowchart programs. In SAS'10 (LNCS 6337). 117-133.

[7] Diego E. Alonso-Blas and Samir Genaim. 2012. On the limits of the classical approach to cost analysis. In SAS'12 (LNCS 7460). 405-421.

[8] Roberto Bagnara, Andrea Pescetti, Alessandro Zaccagnini, and Enea Zaffanella. 2005. PURRS: Towards computer algebra support for fully automatic worst-case complexity analysis. CoRR abs/cs/0512056 (2005).

[9] Amir M. Ben-Amram and Samir Genaim. 2014. Ranking functions for linear-constraint loops. f. ACM 61, 4 (2014), 26:1-26:55.

[10] Régis Blanc, Thomas A. Henzinger, Thibaud Hottelier, and Laura Kovács. 2010. ABC: Algebraic bound computation for loops. In LPAR'10 (LNCS 6355). 103-118.

[11] Marius Bozga, Codruta Gîrlea, and Radu Iosif. 2009. Iterating octagons. In TACAS’09 (LNCS 5505). $337-351$.

[12] Marius Bozga, Radu Iosif, and Filip Konecný. 2010. Fast acceleration of ultimately periodic relations. In CAV'10 (LNCS 6174). 227-242.

[13] Aaron R. Bradley, Zohar Manna, and Henny B. Sipma. 2005. Linear ranking with reachability. In CAV'05 (LNCS 3576). 491-504.

[14] Marc Brockschmidt, Fabian Emmes, Stephan Falke, Carsten Fuhs, and Jürgen Giesl. 2016. Analyzing runtime and size complexity of integer programs. ACM Trans. Prog. Lang. Syst. 38, 4 (2016), 13:1-13:50.

[15] Jacob Burnim, Sudeep Juvekar, and Koushik Sen. 2009. WISE: Automated test generation for worst-case complexity. In ICSE'09. 463-473.

[16] Rod M. Burstall and John Darlington. 1977. A transformation system for developing recursive programs. $\mathcal{~ f . ~ A C M ~ 2 4 , ~}$ 1 (1977), 44-67.

[17] Pavel Cadek, Clemens Danninger, Moritz Sinn, and Florian Zuleger. 2018. Using loop bound analysis for invariant generation. In FMCAD’18. 1-9.

[18] Peter Cameron. 2017. Polynomials Taking Integer Values. Retrieved from https://cameroncounts.wordpress.com/ 2017/01/31/polynomials-taking-integer-values/.

[19] Quentin Carbonneaux, Jan Hoffmann, and Zhong Shao. 2015. Compositional certified resource bounds. In PLDI'15. 467-478.

[20] Quentin Carbonneaux, Jan Hoffmann, Thomas W. Reps, and Zhong Shao. 2017. Automated resource analysis with Coq proof objects. In CAV'17 (LNCS 10427). 64-85.

[21] Leonardo de Moura and Nikolaj Bjørner. 2008. Z3: An efficient SMT solver. In TACAS’08 (LNCS 4963). 337-340. 
[22] Saumya Debray, Pedro López-García, Manuel V. Hermenegildo, and Nai-Wei Lin. 1997. Lower bound cost estimation for logic programs. In ILPS'97. 291-305.

[23] Complexity Analysis-Based Guaranteed Execution. 2015. Retrieved from https://www.draper.com/news-releases/ drapers-cage-could-spot-code-vulnerable-denial-service-attacks.

[24] Stephan Falke, Deepak Kapur, and Carsten Sinz. 2012. Termination analysis of imperative programs using bitvector arithmetic. In VSTTE'12 (LNCS 7152). 261-277.

[25] Azadeh Farzan and Zachary Kincaid. 2015. Compositional recurrence analysis. In FMCAD'15. 57-64.

[26] Antonio Flores-Montoya and Reiner Hähnle. 2014. Resource analysis of complex programs with cost equations. In APLAS'14 (LNCS 8858). 275-295.

[27] Antonio Flores-Montoya. 2016. Upper and lower amortized cost bounds of programs expressed as cost relations. In FM'16 (LNCS 9995). 254-273.

[28] Space/Time Analysis for Cybersecurity (STAC). 2015. Retrieved from http://www.darpa.mil/program/space-timeanalysis-for-cybersecurity.

[29] Florian Frohn, Matthias Naaf, Jera Hensel, Marc Brockschmidt, and Jürgen Giesl. 2016. Lower runtime bounds for integer programs. In IFCAR'16 (LNAI 9706). 550-567.

[30] Florian Frohn, Jürgen Giesl, Jera Hensel, Cornelius Aschermann, and Thomas Ströder. 2017. Lower bounds for runtime complexity of term rewriting. F. Autom. Reas. 59, 1 (2017), 121-163.

[31] Florian Frohn and Jürgen Giesl. 2019. Termination of triangular integer loops is decidable. In CAV'19 (LNCS 11562). 426-444.

[32] Florian Frohn and Jürgen Giesl. 2019. Proving non-termination via loop acceleration. In FMCAD'19. 221-230.

[33] Florian Frohn, Matthias Naaf, Marc Brockschmidt, and Jürgen Giesl. 2020. Empirical Evaluation of "Inferring Lower Runtime Bounds for Integer Programs.” Retrieved from https://aprove-developers.github.io/its-lowerbounds-journal.

[34] Hongfei Fu and Krishnendu Chatterjee. 2019. Termination of nondeterministic probabilistic programs. In VMCAI'19 (LNCS 11388). 468-490.

[35] Pierre Ganty, Radu Iosif, and Filip Konecný. 2017. Underapproximation of procedure summaries for integer programs. STTT 19, 5 (2017), 565-584.

[36] Jürgen Giesl, Peter Giesl, and Marcel Hark. 2019. Computing expected runtimes for constant probability programs. In CADE'19 (LNAI 11716). 269-286.

[37] Jürgen Giesl, Albert Rubio, Christian Sternagel, Johannes Waldmann, and Akihisa Yamada. 2019. The termination and complexity competition. In TACAS'19 (LNCS 11429). 156-166.

[38] Laure Gonnord and Nicolas Halbwachs. 2006. Combining widening and acceleration in linear relation analysis. In SAS'06 (LNCS 4134). 144-160.

[39] Sumit Gulwani, Krishna K. Mehra, and Trishul M. Chilimbi. 2009. SPEED: Precise and efficient static estimation of program computational complexity. In POPL'09. 127-139.

[40] Marcel Hark, Benjamin Lucien Kaminski, Jürgen Giesl, and Joost-Pieter Katoen. 2020. Aiming low is harder-Inductive proof rules for lower bounds on weakest preexpectations in probabilistic program verification. PACMPL 4, POPL (2020), 37:1-37:28.

[41] André Heck. 1996. Introduction to Maple (2nd ed.). Springer.

[42] Jera Hensel, Jürgen Giesl, Florian Frohn, and Thomas Ströder. 2018. Termination and complexity analysis for programs with bitvector arithmetic by symbolic execution. F. Log. Algor. Meth. Prog. 97 (2018), 105-130.

[43] Nao Hirokawa and Georg Moser. 2008. Automated complexity analysis based on the dependency pair method. In IFCAR'08 (LNAI 5195). 364-379.

[44] Dieter Hofbauer and Clemens Lautemann. 1989. Termination proofs and the length of derivations. In RTA'89 (LNCS 355). $167-177$.

[45] Jan Hoffmann, Klaus Aehlig, and Martin Hofmann. 2012. Multivariate amortized resource analysis. ACM Trans. Prog. Lang. Syst. 34, 3 (2012), 14:1-14:62.

[46] Jan Hoffmann and Zhong Shao. 2015. Type-based amortized resource analysis with integers and arrays. f. Funct. Prog. 25 (2015).

[47] Jan Hoffmann, Ankush Das, and Shu-Chun Weng. 2017. Towards automatic resource bound analysis for OCaml. In POPL'17. 359-373.

[48] Bertrand Jeannet, Peter Schrammel, and Sriram Sankaranarayanan. 2014. Abstract acceleration of general linear loops. In POPL'14. 529-540.

[49] KoAT Benchmarks 2014. Retrieved from https://github.com/s-falke/kittel-koat/tree/master/koat-evaluation/ examples.

[50] Daniel Kroening, Matt Lewis, and Georg Weissenbacher. 2015. Under-approximating loops in C programs for fast counterexample detection. Form. Meth. Syst. Des. 47, 1 (2015), 75-92. 
[51] Daniel Larraz, Albert Oliveras, Enric Rodríguez-Carbonell, and Albert Rubio. 2013. Proving termination of imperative programs using Max-SMT. In FMCAD'13. 218-225.

[52] LoAT. 2019. Retrieved from https://github.com/aprove-developers/LoAT.

[53] Kumar Madhukar, Björn Wachter, Daniel Kroening, Matt Lewis, and Mandayam K. Srivas. 2015. Accelerating invariant generation. In FMCAD'15. 105-111.

[54] Annabelle McIver and Carroll Morgan. 2005. Abstraction, Refinement and Proof for Probabilistic Systems. Springer.

[55] Matthias Naaf, Florian Frohn, Marc Brockschmidt, Carsten Fuhs, and Jürgen Giesl. 2017. Complexity analysis for term rewriting by integer transition systems. In FroCoS'17 (LNAI 10483). 132-150.

[56] Andreas Podelski and Andrey Rybalchenko. 2004. A complete method for the synthesis of linear ranking functions. In VMCAI'04 (LNCS 2937). 239-251.

[57] Moritz Sinn, Florian Zuleger, and Helmuth Veith. 2017. Complexity and resource bound analysis of imperative programs using difference constraints. f. Autom. Reas. 59, 1 (2017), 3-45.

[58] Akhilesh Srikanth, Burak Sahin, and William R. Harris. 2017. Complexity verification using guided theorem enumeration. In POPL'17. 639-652.

[59] Jan Strejcek and Marek Trtík. 2012. Abstracting path conditions. In ISSTA'12. 155-165.

[60] Di Wang and Jan Hoffmann. 2019. Type-guided worst-case input generation. PACMPL 3, POPL (2019), 13:1-13:30.

[61] Stephen Wolfram. 1992. Mathematica: A system for doing mathematics by computer. SIAM Rev. 34, 3 (1992), 519-522.

Received September 2019; revised June 2020; accepted July 2020 